\title{
Predictors and Health Outcomes of Treatment-Resistant Depression among Adults with Chronic Non-Cancer Pain Conditions and Major Depressive Disorder
}

\author{
Drishti Shah \\ West Virginia University, drshah@mix.wvu.edu
}

Follow this and additional works at: https://researchrepository.wvu.edu/etd

Part of the Clinical Psychology Commons, Epidemiology Commons, Mental Disorders Commons, Musculoskeletal Diseases Commons, Pain Management Commons, Pharmacoeconomics and

Pharmaceutical Economics Commons, and the Psychiatric and Mental Health Commons

\section{Recommended Citation}

Shah, Drishti, "Predictors and Health Outcomes of Treatment-Resistant Depression among Adults with Chronic Non-Cancer Pain Conditions and Major Depressive Disorder" (2019). Graduate Theses, Dissertations, and Problem Reports. 3855.

https://researchrepository.wvu.edu/etd/3855

This Dissertation is protected by copyright and/or related rights. It has been brought to you by the The Research Repository @ WVU with permission from the rights-holder(s). You are free to use this Dissertation in any way that is permitted by the copyright and related rights legislation that applies to your use. For other uses you must obtain permission from the rights-holder(s) directly, unless additional rights are indicated by a Creative Commons license in the record and/ or on the work itself. This Dissertation has been accepted for inclusion in WVU Graduate Theses, Dissertations, and Problem Reports collection by an authorized administrator of The Research Repository @ WVU.

For more information, please contact researchrepository@mail.wvu.edu. 


\title{
Predictors and Health Outcomes of Treatment-Resistant Depression
} among Adults with Chronic Non-Cancer Pain Conditions and Major Depressive Disorder

\author{
Drishti Shah \\ Dissertation submitted \\ to the School of Pharmacy \\ at West Virginia University
}

in partial fulfillment of the requirements for the degree of

Doctor of Philosophy in

Health Services and Outcomes Research

\author{
Usha Sambamoorthi, Ph.D, Chair \\ Suresh Madhavan, Ph.D \\ Traci J. LeMasters, Ph.D. \\ Lindsay Allen, Ph.D. \\ Wenhui Wei, Ph.D. \\ Department of Pharmaceutical Systems and Policy \\ Morgantown, West Virginia \\ 2019
}

Keywords: Treatment-resistant depression, major depressive disorder, chronic non-cancer pain conditions, predictors, healthcare costs, healthcare resource utilization, long-term opioid therapy

Copyright 2019 Drishti Shah 


\section{ABSTRACT \\ Predictors and Health Outcomes of Treatment-Resistant Depression among Adults with Chronic Non-Cancer Pain Conditions and Major Depressive Disorder}

\section{Drishti Shah}

Understanding major depressive disorder (MDD) as a comorbidity in patients with chronic noncancer pain conditions (CNPC) is of importance because of the high prevalence and well documented bi-directional relationship between MDD and pain. Furthermore, presence of CNPC among adults with MDD often reduces benefits of antidepressant therapy, thereby increasing the possibility of treatment resistance. Treatment-resistant depression (TRD) commonly defined as insufficient response to multiple antidepressant trials, often worsens depression and pain symptoms and can amplify the clinical and economic burden among adults with CNPC and MDD. Additionally, long-term opioid therapy (LTOT) may be prescribed at a higher rate to adults with TRD to treat an undifferentiated state of physical and mental pain, despite lack of evidence on LTOT use in this patient population. Existing literature on TRD has focused on all adults with MDD. Owing to the lack of research on TRD among adults with CNPC and MDD, we conducted this study to fill a critical knowledge gap. This dissertation pursued three related aims: 1) identify leading predictors of TRD; 2) estimate the direct economic burden associated with TRD and identify factors that contribute to the excess cost burden of TRD; and 3) examine the trajectory of LTOT use in adults with CNPC and newly diagnosed MDD and how TRD can alter this trajectory. The study used a retrospective, longitudinal, cohort design, using multiple years (2007-2017) of the de-identified, geographically diverse data on commercially insured adults obtained from the $10 \%$ random sample of Optum ${ }^{\circledR}$ Clinformatics ${ }^{\circledR}$ Data Mart. TRD was identified using a comprehensive claims-based algorithm. Among adults (>18 years) with CNPC and newly diagnosed MDD who were treated with antidepressants $(\mathrm{N}=23,645)$, approximately one in nine adults transitioned to TRD within a year of MDD diagnosis. First, we identified the use of mental health specialist visits, polypharmacy, psychotherapy use, anxiety, and age to be the five leading predictors of TRD using random forest, a machine learning method and crossvalidated logistic regression. In the second aim, we observed that patients with TRD had

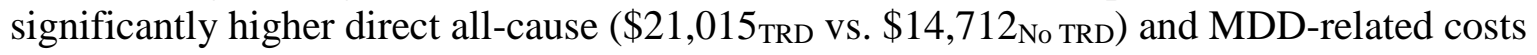

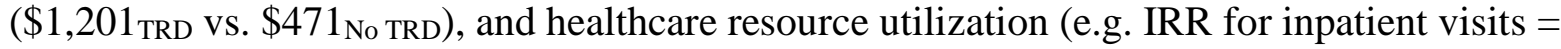
1.30. $95 \% \mathrm{CI}=1.14-1.47 ; \mathrm{P}<0.001$ ) as compared to those without TRD. Majority of excess total healthcare costs among adults with TRD were driven by differences in rates of polypharmacy between the TRD and non-TRD groups. Lastly, findings from the third aim indicated that adults with TRD were more likely to receive LTOT [AOR: 1.54, 95\% CI: 1.68-2.04), although LTOT use among TRD group declined over the three-year observation period, at a higher rate than those without TRD $(\beta=-.033, \mathrm{SE}=0.012)$. In summary, these findings underscore the need for effective, evidence-based strategies to manage depression and pain among adults with TRD and CNPC. In addition, the current study identified modifiable factors that warrant further investigation and may serve as potential targets for clinical and policy interventions to reduce the risk of TRD and off-set the excess costs associated with TRD. Furthermore, high rates of LTOT use among adults with TRD suggest that clinicians need to closely monitor the risks of using LTOT in this subgroup. 


\section{DEDICATION}

I dedicate this dissertation to my mother, Rupal Shah, for always being a constant source of motivation. She's the strongest woman I know. Her strength, unconditional love and support have always inspired me to my very best. 
As I reflect on the last three years, I take this moment to thank and express my deepest gratitude to the people who have been instrumental to this project and in shaping my life. First and foremost, I would like to express the deepest appreciation to my major advisor, mentor, and guide Dr. Usha Sambamoorthi for her relentless guidance, support, and encouragement to pursue my dissertation idea, and in helping me translate my idea into a full-fledge dissertation that you see today. I am truly blessed to have a professor and mentor like Dr. Sambamoorthi. She is one of the most intelligent and humble persons that I know and her teachings through coursework and projects have immensely benefited me in advancing my methodological, technical, and reasoning skills. Without her constant oversight, feedback, and guidance, I would not have been able to complete this dissertation. Words fail me in expressing the depth of respect that I have for her. I believe that if I can even achieve $30 \%$ of the efficiency that she has in executing her projects, after adjusting for characteristics such as intellectual laziness, technical skills, and leisure time, I will consider myself to be a good health outcomes researcher.

I would also like to extend my sincere gratitude to Dr. Suresh Madhavan, Dr. Traci LeMasters, Dr. Lindsay Allen, and Dr. Wenhui Wei for their time, patience, valuable suggestions, and guidance during the dissertation process. I would like to thank Dr. Madhavan for being the most wonderful department chair and for time and again providing valuable life advice. I am grateful to Dr. LeMasters for providing timely feedback and for helping me improve my writing skills. Dr. Allen: thank you for your prompt feedback, motivational emails and suggestions on policy implications. Dr. Wei: I cannot thank you enough for all the numerous opportunities that you have provided to me over the years; you have truly played a critical role in grooming me as a researcher and a critical thinker. Special thanks to Dr. Wanhong Zheng for 
providing helpful clinical insights and valuable feedbacks despite his busy schedule. The experience of joining him on clinical rotations helped me put this study into perspective.

I am extremely thankful to West Virginia University, and all the faculty, staff, and students of the PSP department for providing me the necessary support to succeed. I also want to acknowledge my wonderful colleagues, roommates and friends, Xiaohui Zhao and Pragya Rai for being my biggest cheerleaders, for working through days and nights on projects with me before deadlines, and for giving me fond memories that I will always cherish. Thank you, Xiaohui for being my partner in crime to tackle the new data. I would like to further extend thanks to Pragya, Jayesh, Himaly, and other PSP graduate students for their constant support and making my stay in Morgantown joyous and memorable.

Last but not the least, I would like to acknowledge my parents and family for their unconditional love and support which helped me throughout my life, especially in the last one year. I am extremely thankful to my mom, dad, aunt, and uncle for all the sacrifices they made for me to make me what I am today and to my sisters, Bhoomi and Khyati for always motivating and believing in me. I am also truly blessed to have the support and understanding of my husband, Nisarg Shah. I cannot thank him enough for his patience, the numerous jokes he cracked, and the desserts he sent to uplift my mood during stressful times. Nisarg has been so understanding and always made me believe that I can do this, and it will be worth it every time I had a long day at work. I am extremely thankful to my beautiful extended family, my parents and sister-in-law for their love and support and to not make me feel like I am thousands of miles away from my family. I would also like to thank my cousins, friends, and relatives for looking out for me and for understanding when I was unable to physically be a part of major live events that took place in their lives over the past few years. 


\section{Table of Contents}

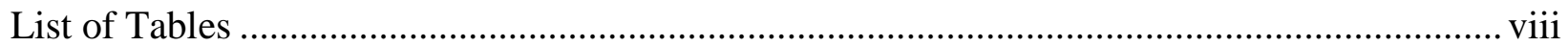

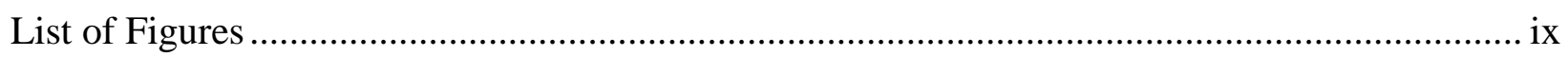

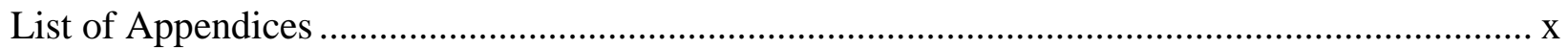

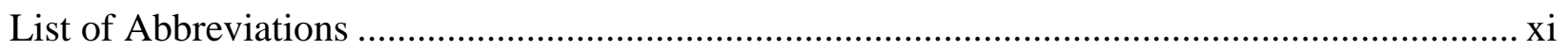

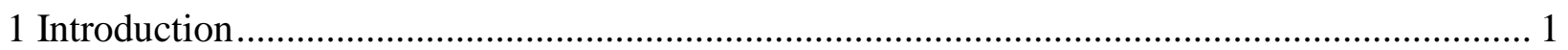

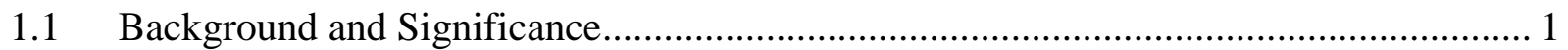

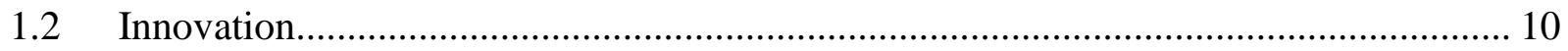

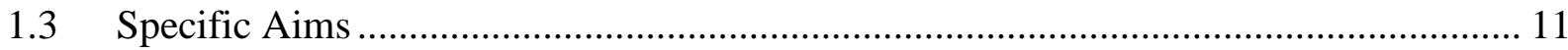

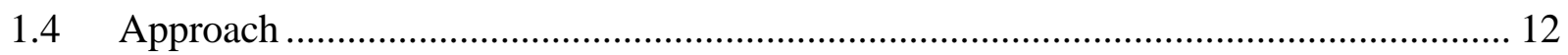

2 Predictors of Treatment-Resistant Depression among Adults with Chronic Non-Cancer Pain Conditions and Major Depressive Disorder: A Machine Learning Approach ............................. 16

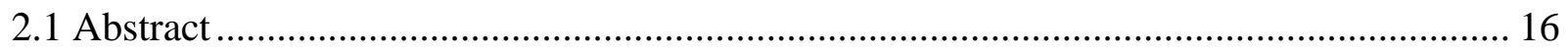

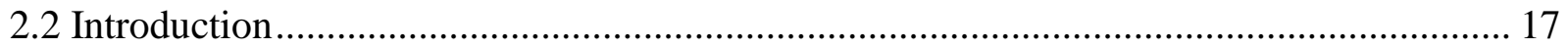

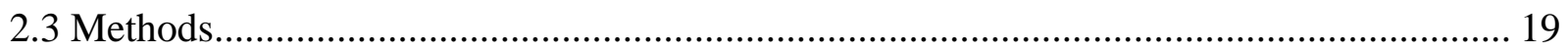

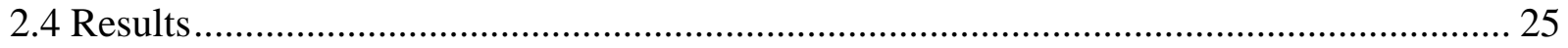

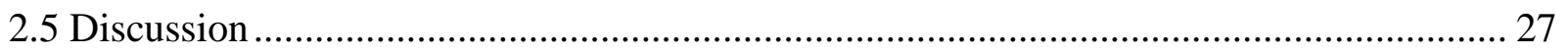

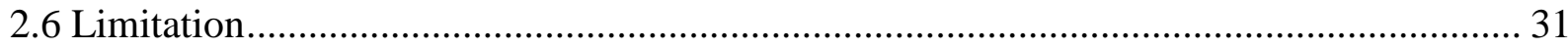

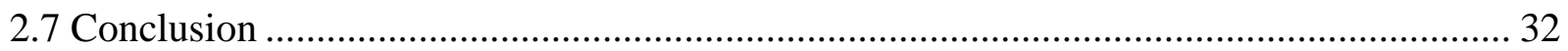

3 Economic Burden of Treatment-Resistant Depression among Adults with Chronic Non-Cancer

Pain Conditions and Major Depressive Disorder................................................................... 40

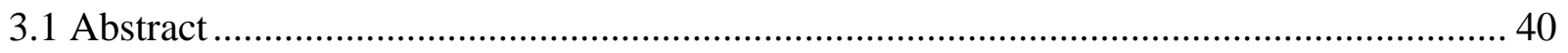

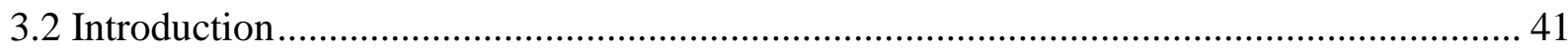

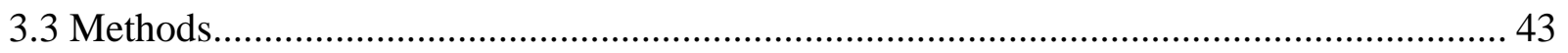


3.4 Results......

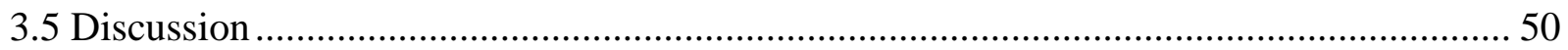

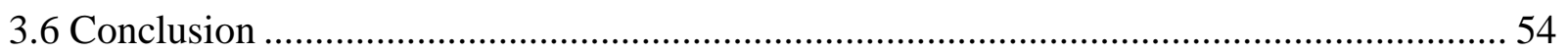

4 Trajectories of Long-term Opioid Use: The Role of Treatment-Resistant Depression among Adults with Chronic Non-Cancer Pain Conditions and Newly Diagnosed Major Depressive

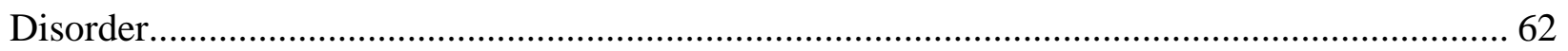

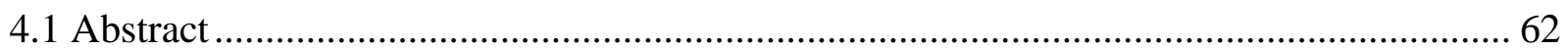

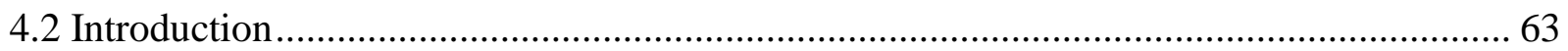

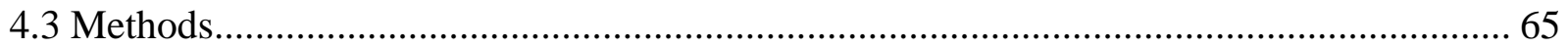

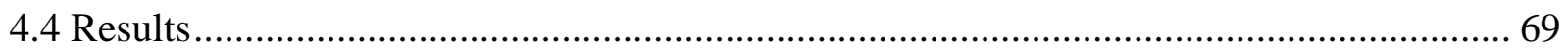

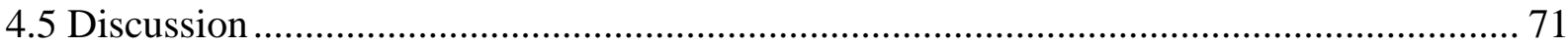

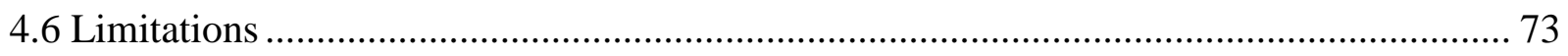

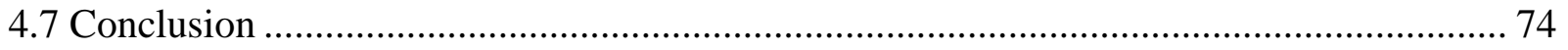

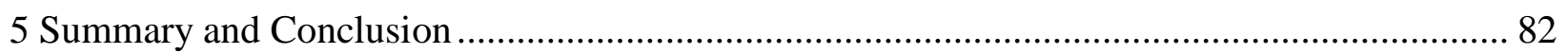

5.1 Summary of Findings and Discussion ............................................................... 82

5.2 Implications and Suggestions for Future Research............................................... 85

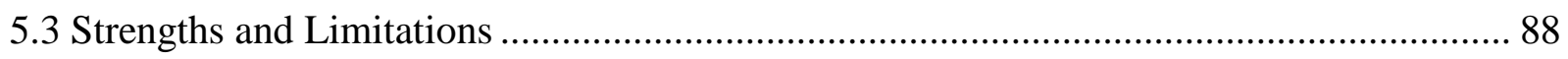

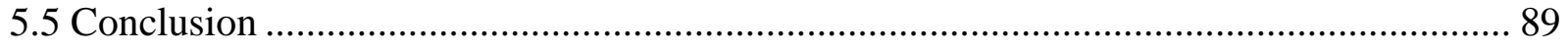

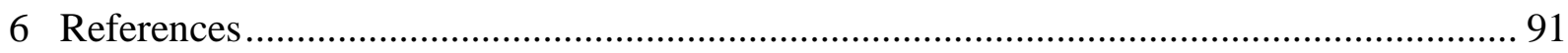

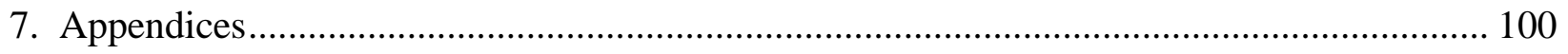




\section{List of Tables}

Table 2.1 List of All 42 Predictors Considered for Model Inclusion Described by the Conceptual

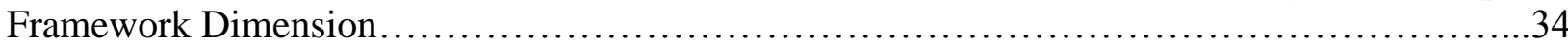

Table 2.2 Description of Adults with CNPC and Newly Diagnosed MDD by TRD, Optum Clinformatics Real-World De-identified Data, 2007-2017.

Table 2.3 Adjusted Odds Ratio and 95\% Confidence Intervals for Important Predictors of TRD from Logistic Regression Model for Adults with CNPC and Newly Diagnosed MDD, Optum Clinformatics Real-World De-identified Data, 2007-2017 39

Table 3.1 Baseline Characteristics of Adults with CNPC and Newly Diagnosed MDD, Optum Clinformatics Real-World De-identified Data, 2007-2017

Table 3.2 Mean Annual Adjusted and Unadjusted All-Cause and MDD-Related Costs Among Adults with CNPC and Newly Diagnosed MDD by TRD status, Optum Clinformatics DataMart, 2007-2017.

Table 3.3 Post Linear Decomposition to Explain Contribution of Patient Factors on All-Cause Total Healthcare Cost: Differences by TRD Status among Adults with CNPC and Newly Diagnosed MDD Optum Clinformatics DataMart, 2007-2017.

Table 4.1 Overall Baseline Sample Characteristics and Characteristics by use of LTOT among Adults with CNPC and Newly Diagnosed MDD (measured during pre-index period), Optum Clinformatics Real-World De-identified Data, 2007-2017................................77

Table 4.2 Unadjusted and Adjusted Odds Ratio and 95\% Confidence Interval from Generalized Estimating Equation (GEE) of TRD on Long -term opioid therapy among Adults with CNPC and Newly Diagnosed MDD, Optum Clinformatics Real-World De-identified Data, 2007-2017...80 


\section{List of Figures}

Figure 1.1 Adapted determinants of health outcomes and chronic disease model...............13

Figure 1.2 Expanded Symptom Management Model....................................... 15

Figure 2.1 Schematic presentation of study sample selection-Inclusion and exclusion criteria applied to patients with CNPC and newly diagnosed episode of MDD, Optum Clinformatics Data Mart, observation period January 2007-June 2017.

Figure 2.2 Variable importance values for top 10 variables of TRD among patients with CNPC and newly diagnosed MDD obtained from Machine Learning Approaches: A) Logistic regression B) Random forest models....................................................... 38

Figure 3.1 Schematic presentation of study sample selection..............................56

Figure 3.2 Adjusted average annual healthcare resource utilization associated with TRD among

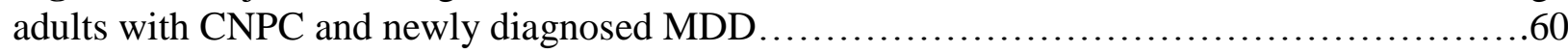

Figure 4.1 Schematic of the study design. ....................................................

Figure 4.2 Schematic presentation of study sample selection..............................76

Figure 4.3 Percentage of LTOT use over time for all adults and by TRD status among adults

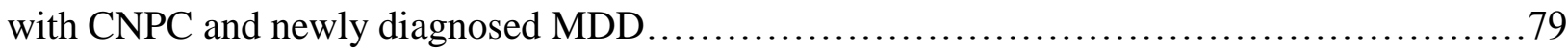




\section{List of Appendices}

Appendix 7.1 ICD-9/10 diagnosis codes used to identify CNPC, MDD and other chronic conditions....

Appendix 7.2 Chapter 2 Study design for predicting treatment resistant depression among adults with CNPC and newly diagnosed MDD

Appendix 7.3 Chapter 2 Representation of development and testing of machine learning model.

Appendix 7.4 Chapter 2 Components of the Algorithm for identification of patients with TRD.....

Appendix 7.5 Chapter 2 Performance measures for the prediction models

Appendix 7.6 Chapter 3 Baseline patient characteristics of adults with CNPC and Newly Diagnosed MDD by TRD status before and after Inversed Probability of Treatment Weighting (IPTW), Optum Clinformatics DataMart, 2007-2017.

Appendix 7.7 Chapter 3 Mean Annual IPTW Adjusted and Unadjusted All-Cause and MDDRelated Costs Among Adults with CNPC and Newly Diagnosed MDD, Optum Clinformatics DataMart, 2007-2017.

Appendix 7.8 Chapter 3 Mean Annual Adjusted and Unadjusted All-Cause and MDD-Related Utilization Among Adults with CNPC and Newly Diagnosed MDD Treated with Antidepressants by TRD status Optum Clinformatics DataMart, 2007-2017.

Appendix 7.9 Chapter 3 Unadjusted and Adjusted All-Cause Total and MDD-related Total Costs over Time among Adults with CNPC and Newly Diagnosed MDD Treated with Antidepressants by TRD status Optum Clinformatics DataMart, 2007-2017. ...

Appendix 7.10 Chapter 4 Components of the Algorithm for identification of patients with TRD.

Appendix 7.11 Chapter 4 Sample Characteristics by TRD Status among Adults with CNPC and Newly Diagnosed MDD, Optum Clinformatics Real-World De-identified Data, 2007-2017...112

Appendix 7.12 Chapter 4 Percentage of LTOT use (defined as continuous opioid supply for $>120$ days) over time for all adults and by TRD status among adults with CNPC and newly diagnosed MDD.

Appendix 7.13 Chapter 4 Unadjusted and Adjusted Odds Ratio and 95\% Confidence Interval from Generalized Estimating Equation (GEE) of TRD on Long -term opioid therapy (defined as continuous opioid supply for $>120$ days) among Adults with CNPC and Newly Diagnosed MDD, Optum Clinformatics Real-World De-identified Data, 2007-2017. 


\section{List of Abbreviations}

\begin{tabular}{|c|c|}
\hline AOR & Adjusted odds ratio \\
\hline AUC & Area Under the Curve \\
\hline $\mathrm{CI}$ & Confidence interval \\
\hline $\mathrm{COL}$ & Column \\
\hline CNPC & Chronic Non-Cancer Pain Conditions \\
\hline COPD & Chronic Obstructive Pulmonary Disease \\
\hline $\mathrm{CPT}$ & Current Procedural Terminology \\
\hline $\mathrm{ED}$ & Emergency Department \\
\hline $\mathrm{EPO}$ & Exclusive Provider Organization \\
\hline FFS & Fee-for-service \\
\hline GEE & Generalized Estimating Equations \\
\hline GLM & Generalized Linear Model \\
\hline GPO & Group Provider Organization \\
\hline HCPCS & The Healthcare Common Procedure Coding System \\
\hline HMO & Health Maintenance Organization \\
\hline HRU & Healthcare Resource Utilization \\
\hline ICD-9/10-CM & $\begin{array}{l}\text { International Classification of Diseases, Ninth/Tenth Revision, Clinical } \\
\text { Modification }\end{array}$ \\
\hline IP & Inpatient \\
\hline IPTW & Inverse Probability of Treatment Weighting \\
\hline IRR & Incidence Rate Ratios \\
\hline LR & Logistic Regression \\
\hline LTOT & Long-term Opioid Therapy \\
\hline MAOI & Monoamine Oxidase Inhibitors \\
\hline MGH & Massachusetts General Hospital \\
\hline MDD & Major Depressive Disorder \\
\hline NSAID & Non-steroidal anti-inflammatory drugs \\
\hline OOB & Out of Bag \\
\hline OOP & Out-of-pocket \\
\hline OR & Odds Ratio \\
\hline PCP & Primary care physician \\
\hline PPO & Preferred Provider Organization \\
\hline POS & Point-of-Service \\
\hline $\mathrm{RF}$ & Random Forest \\
\hline $\mathrm{ROC}$ & Receiver Operating Characteristic \\
\hline SD & Standard deviation \\
\hline SE & Standard error \\
\hline SIG & Significance \\
\hline SNRI & Serotonin Norepinephrine Reuptake Inhibitors \\
\hline SSRI & Selective Serotonin Reuptake Inhibitors \\
\hline STAR*D & Sequenced Treatment Alternatives to Relieve Depression \\
\hline TCA & Tricyclic Antidepressants \\
\hline TPM & Two-part Model \\
\hline TRD & Treatment-resistant Depression \\
\hline US & United States \\
\hline
\end{tabular}




\section{CHAPTER 1}

\section{Introduction}

\subsection{Background and Significance}

\section{Epidemiology of Major Depressive Disorder}

Major Depressive Disorder (MDD) is the leading cause of disability worldwide, and is a major contributor to the burden of suicide. ${ }^{1} \mathrm{MDD}$ is highly prevalent and affects approximately 35 million adults in the United States (US). ${ }^{2,3}$ Among adults in US, MDD accounts for nearly 3.7 percent of all disability-adjusted life years (DALYs) and 8.3 percent of all years lived with disability (YLDS). ${ }^{4}$ MDD disproportionately affects women; women are $70 \%$ more likely to experience MDD during their lifetime than men. As per the National Comorbidity Study, the lifetime prevalence of MDD in the US was estimated to be $21.3 \%$ in women and $12.7 \%$ in men. ${ }^{5}$ It has been reported that MDD is significantly associated with increased risk of other psychiatric disorders, notably anxiety disorders, substance use disorders and other chronic conditions such as chronic non-cancer pain conditions (CNPC) ${ }^{2,}$, 6 MDD also plays a significant role in worsening functional status, with the greatest impairment in the social and cognitive domains. ${ }^{2}$

\section{Burden of MDD}

MDD is associated with significant clinical, humanistic, and economic burden. ${ }^{1,8}$ It is projected to cause the greatest illness burden in high-income countries by $2030 .{ }^{9}$ In 2015 , the incremental economic burden of MDD in the US was estimated to be as high as $\$ 210.5$ billion, with $\$ 98.9$ billion spent on direct healthcare costs. ${ }^{8}$ Co-existence of MDD and other chronic conditions has been reported to be the main driver of this high economic burden associated with MDD. ${ }^{8}$ For example, MDD is associated with $50 \%$ to $100 \%$ higher medical costs among patients with co-existing chronic conditions even after risk adjustments. ${ }^{10}$ Among commonly co- 
occurring chronic conditions with MDD, chronic non-cancer pain conditions (CNPC) is of particular interest because of the high prevalence and well documented bi-directional relationship between MDD and pain. ${ }^{7,11}$

\section{MDD among Adults with Chronic Non-cancer Pain Conditions}

Of all the chronic conditions that can co-exist or develop in MDD patients, commonly occurring CNPC including, back/neck pain, headache/migraine, and neuropathic pain conditions deserve special attention because of the special pathophysiology between MDD and CNPC.

\section{Link between MDD and CNPC}

MDD and painful symptoms, commonly experienced by adults with CNPC often cooccur together. ${ }^{7,12}$ MDD and its treatment can be affected by pain, which is also considered as a somatic symptom of depression. ${ }^{13}$ A growing body of literature has documented the bidirectional relationship between chronic pain and MDD. As pain and MDD are inextricably linked in terms of the shared biological pathways and neurotransmitters, they often respond to similar treatments, and exacerbate one another. Some researchers have labeled this relationship as depression-pain syndrome. ${ }^{14,15}$ It has been suggested that one of the important causes for chronic pain leading to depression and vice versa appears to be the common neuroplasticity changes shared by pain and depression such as changes in monoamine neurotransmitters, brainderived neurotrophic factor, inflammatory factors, and glutamate functions. ${ }^{16}$

\section{Prevalent MDD among Adults with CNPC}

Given that pain is often experienced by adults with CNPC, several studies have examined the prevalence of MDD in patients with CNPC. A narrative review on MDD and pain summarized the prevalence of MDD in adults with CNPC and reported that the estimated prevalence of MDD in patients with CNPC can be as high as $85 \% .{ }^{7}$ However, depending on the 
type of condition and setting, ${ }^{7}$ MDD prevalence can range from $38 \%$ in psychiatric clinics, $52 \%$ in pain clinics, $56 \%$ in orthopedic or rheumatology clinics, and to $85 \%$ in dental clinics. ${ }^{7}$

\section{Treatment-Resistant Depression among Adults with MDD}

A significant proportion of the economic and clinical burden of MDD is disproportionately accounted by individuals who do not achieve recovery from the illness. Consensus exists that a majority of individuals (nearly 50\%) do not achieve complete remission with index antidepressant therapy. The designation -"treatment-resistant depression (TRD)" is primarily used to describe patients who do not respond to more than two adequate trials of antidepressants. ${ }^{17-20}$ The STAR*D trial, the largest and longest study ever conducted to evaluate the effectiveness of depression treatment reported that even after undergoing four sequential antidepressant trials, $30 \%$ of patients did not achieve remission. ${ }^{21}$ Due to the variations in TRD definitions in studies using administrative claims or similar datasets, the prevalence of TRD has ranged from $6 \%{ }^{22}$ to $30 \% .^{23}$

\section{High risk of TRD among Adults with CNPC and MDD}

CNPC may contribute to TRD among adults with MDD. Biar et al investigated the presence of pain conditions on treatment response to depression in primary care. ${ }^{11}$ They concluded that the presence of baseline CNPC among adults with MDD reduces the benefits of antidepressant therapy at 12 weeks, suggesting treatment resistance. ${ }^{11}$ Indirect evidence of the relationship between CNPC and TRD has been reported. A Canadian Study by Rizvi et al. and a US study by Kubitz et al. reported that the prevalence of CNPC such as joint pain, muscle pain, headache/migraine, and back pain was two times higher among patients with TRD as compared to those without TRD. ${ }^{22,24}$ It is plausible that adults with CNPC may be at higher risk of having TRD, because pain experienced by those with CNPC may reduce the efficacy of antidepressant 
therapy. ${ }^{11}$ However, to date, no study has systematically examined the risk of TRD among adults with CNPC and MDD, suggesting a significant need for studies on TRD among adults with CNPC. In this context, the first critical step in filling this knowledge gap is an examination of risk factors that influence the transition from MDD to TRD among adults with CNPC.

\section{Risk Factors for TRD among Adults with CNPC and MDD}

TRD can be influenced by biological, health- and treatment- related factors. In studies not specific to CNPC, it has been reported that women (71\%), non-Hispanic whites (89\%), adults with comorbid conditions (for example, CNPC, cardiovascular diseases, hypertension, and other psychiatric conditions), obesity, highly activated inflammatory system, and patients who are hospitalized were more likely to experience TRD compared to those without these risk factors. 20,22,24,25 Recent studies conducted using predictive models to identify risk factors of TRD using data obtained through the Group for the Study of Resistant Depression (GSRD), across participating centers in Europe concluded that depression severity, age, time span between first and last depressive episodes, suicidal risk, body mass index, number of past depressive episodes, and lifetime duration of hospitalizations were important predictors of TRD. ${ }^{26-28}$ It has to be noted that these studies included prevalent cases of MDD and therefore, the identified factors may not be plausible risk factors for TRD among patients with newly diagnosed MDD. Another study that sought to find risk factors of TRD among patients with newly diagnosed MDD reported that patients with TRD were more likely to be younger, have a history of substance use disorders,

psychiatric conditions, insomnia, and pain as compared to non-TRD patients. ${ }^{29}$ However, to date, no study has identified risk factors of TRD among adults with CNPC and newly diagnosed MDD. 
As the current study focused on adults with CNPC and MDD, in addition to the above factors, association of treatment factors with TRD, such as pain therapy commonly used for CNPC needs to be investigated. It has been reported that effective pain treatment can relieve depressive symptoms ${ }^{36,37}$ and may therefore reduce the risk of TRD. On the other hand, type of pain treatment can positively or negatively impact TRD. For example, opioid use may increase the risk of TRD. A retrospective study on the dose and duration of opioid therapy on TRD among Veterans reported that longer duration of opioid treatment increased the risk of transitioning from MDD to TRD. ${ }^{30}$ Pain treatment with nonsteroidal anti-inflammatory drugs (NSAIDs) may improve depression outcomes among adults with specific CNPC such as osteoarthritis. ${ }^{31,32}$ As the long term use of opioids in patients with CNPC is not effective, ${ }^{33,34}$ there is critical a need to assess the association of other pain therapies such as the use of NSAIDs, and other non-pharmacological pain therapies such as psychotherapy with TRD. Since psychotherapies such as cognitive behavioral therapies are effective for treating both MDD and chronic pain, they may reduce the risk of TRD. ${ }^{35}$

The current study also included factors that have not been examined in other studies such as polypharmacy. It has been documented that the concurrent use of 3 or more medications with depression as an adverse event may increase the risk of having concurrent MDD. ${ }^{36}$ Additionally, certain commonly used medications (e.g. benzodiazepines, corticosteroids, and beta-blockers, and certain muscle relaxants) have higher rates of drug-drug interactions with antidepressants and dilute plasma concentration of antidepressants, which may lead to poor clinical response and TRD. ${ }^{37}$ Therefore, polypharmacy as a predictor of TRD needs to be investigated.

Early identification of leading predictors of TRD in real-world clinical settings with robust statistical and machine leaning methods can inform targeted treatment approaches. As 
these predictive models require a large of number of observations, population-based claims data can be ideal. ${ }^{38,39}$ This leads to the rationale for Aim 1, which examined the risk factors of transitioning from MDD to TRD among adults with CNPC and newly diagnosed MDD.

\section{Role of TRD in Amplifying Burden of MDD}

A review on the disease burden of TRD highlighted that the number of medicationrelated adverse events were higher and the quality of life scores were 26 points lower for adults with TRD when compared to those adults with MDD who experienced remission. ${ }^{20}$ Additionally, adults with MDD also incur a higher economic burden due to TRD. Those with TRD have an excess annual direct healthcare cost of $\$ 5,461$ per patient and an additional $\$ 4,048$ per patient in productivity losses. Extrapolating these costs to national level yielded an estimated \$29-\$46 billion due to TRD (depending on the prevalence estimates of 12 and 20 percent respectively), thereby pushing up the total societal costs of MDD to as high as $\$ 106-\$ 118$ billion. ${ }^{20}$ The burden of TRD is on par with or even higher than other medical conditions such as cancer and diabetes, yet MDD ranks $15^{\text {th }}$ among conditions that receive funding from the National Institutes of Health, ${ }^{40}$ thereby underscoring the need for more research on TRD.

\section{Economic Burden of TRD}

Nearly one-third of the excess cost of MDD has been attributed to TRD. ${ }^{20}$ Among working-age adults with MDD, several studies have quantified the direct healthcare utilization

and costs associated with TRD. ${ }^{22,23,25,30,41-45}$ Although the reported incremental economic burden of TRD varied depending on the definition of TRD and type of study setting; on an average, working age adults with TRD had two times higher direct healthcare costs as compared to those without TRD. ${ }^{23,25,41,42}$ A recent study that estimated the cost burden of TRD reported that adults with TRD incurred $\$ 6,709$ and $\$ 9,917$ higher per patient per year costs as compared to non-TRD 
adults with MDD and non-MDD patients. ${ }^{46}$ Furthermore, those with TRD also had significantly higher odds of using inpatient, outpatient and ER services. ${ }^{23,25,22}$ However, none of these existing studies have estimated the economic burden of TRD among adults with CNPC. A population based study that did not focus on TRD concluded that the presence of concomitant pain conditions in patients with MDD initiated 20\% higher visits to medical providers than those with MDD but no pain conditions. ${ }^{47}$ Presence of TRD can further amplify this burden.

Understanding the relationship between TRD and healthcare costs and utilization among a specific subgroup of patients with CNPC can provide meaningful information to payers and policy makers on how patients' MDD and pain-related complexity can affect healthcare resource use. Given limited healthcare resources, healthcare costs have always been an essential measure of health outcomes in the US. In response to the rising healthcare costs, the Institute of Health Improvement developed the "Triple Aim" which requires identification of high-cost, high-need patients. ${ }^{48}$ Therefore, such cost of illness studies are important not only to provide benchmark for evaluation of alternative demands on scarce health care resources, but also help in translating the burden of illness into dollar terms, the universal language for decision making. ${ }^{49}$ As there are no cost estimates on TRD among adults with CNPC and MDD, estimates obtained from the current study can be used as input parameters in future cost-effectiveness studies.

Furthermore, as highlighted above, TRD can be influenced by many factors. ${ }^{20,27,29,50}$ Identification of key contributors of the incremental TRD burden using advanced decomposition methods can assist policy makers in assessing if the limited healthcare resources are being inequitably used and can aid payers in identifying major cost drivers. This leads to the rationale for Aim 2, which estimated the incremental economic burden (direct healthcare utilization and costs) associated with TRD among adults with CNPC and newly diagnosed MDD. In addition, a 
post-regression decomposition approach was adopted to explain patient level factors that may contribute to the difference in total healthcare costs between adults with and without TRD. Role of TRD in the trajectory of pain treatment with opioids among adults with CNPC

As stated before, pain is a hallmark symptom of CNPC and is also a somatic symptom of MDD. ${ }^{7,11}$ It difficult to manage pain among patients with CNPC and MDD for several reasons: 1) patients with co-existing CNPC and MDD report higher pain scores as compared to those with CNPC but no MDD; ${ }^{51}$ 2) increased difficulty in managing chronic pain and MDD may come with patients that also have substance use disorders. For example, it has been reported that patients with MDD are 1.8-2.4 times likely to misuse opioid medications, ${ }^{5} 3$ ) patients with MDD and pain report higher impairment in functional capacity; 4) depression severity increases when pain is more diffuse, as indicated by multisite pain, often experienced by patients with CNPC. ${ }^{52}$

As adults with co-existing CNPC and MDD may experience pain at higher intensity which may not be responsive to first line pain therapies such as NSAIDs, opioids may be prescribed for longer duration. There has been a growing need to examine the risks of long-term opioid therapy (LTOT) among patients with CNPC due to the concern that the safety and effectiveness of LTOT in CNPC remains unproven. ${ }^{53,54}$ Further, no treatment guidelines recommend LTOT for patients with CNPC who also have MDD. ${ }^{55}$ Yet patients with CNPC and MDD continue to receive LTOT in real-world settings. ${ }^{55}$ As MDD often makes chronic pain worse among adults with CNPC, ${ }^{11}$ patients with MDD and chronic pain may often preferentially seek relief for their pain, which may be less stigmatized. Health care providers may be obligated to prescribe medications such as opioids to treat the undifferentiated state of physical and mental pain in these patients. In fact, patients with MDD in real-world are more likely to receive LTOT than those without MDD diagnoses. ${ }^{56}$ 
As patients with TRD experience worse depression symptoms and physical symptoms such as pain as compared to those without TRD, ${ }^{26}$ LTOT may be prescribed at a higher rate among patients with TRD. TRD may increase the use of LTOT $^{55}$ as the neural patterns of activation that are associated with depression are also associated with pain, and LTOT may be prescribed to treat the complex combination of both pain and TRD. ${ }^{55,57}$ In addition, historically opioids have been used to treat psychological distress caused by mental health conditions. ${ }^{5,58}$ LTOT in patients with TRD may further exacerbate the risk of opioid misuse leading to detrimental consequences such as death due to overdose, intentional harm, and suicide. ${ }^{1,5}$ On the other hand, clinicians may be more cautious in prescribing LTOT to MDD patients who develop TRD. This is because this group of patients may be deemed to be a high- risk group as both LTOT and TRD are independently associated with increased risk of substance use disorders and adverse health outcomes. ${ }^{30,3220,22}$ While, there is evidence that patients with MDD and pain are more likely to receive LTOT, ${ }^{56,59,60}$ and LTOT may increase the risk of TRD, ${ }^{30}$ to date, no study has examined the trajectory of LTOT use among patients with CNPC and MDD and how TRD may alter this trajectory.

The central question in prescribing LTOT for CNPC and TRD is how to best balance the risk of opioid abuse and dependence. The first critical step in answering this question is an understanding of the pattern of LTOT prescription among adults with CNPC and MDD and the association of TRD with the trajectory of LTOT use. Robust evidence on the pattern of LTOT use among adults with CNPC and MDD and any efforts to understand the risk factors of LTOT are useful in the broader context of battling the current opioid epidemic. Findings from the current study can inform clinical practice, payers, and policy makers in their surveillance efforts of controlling long- term opioid use and misuse. Therefore, a study examining the trajectory of 
LTOT in adults with CNPC and newly diagnosed MDD and the association of TRD with LTOT is needed, thereby justifying the rationale for aim 3 of the current study.

\subsection{Innovation}

a) Comprehensive evaluation of risk factors associated with TRD among adults with CNPC and newly diagnosed MDD: This is the first study to conduct comprehensive analyses of biological, health- and treatment- related factors associated with TRD among adults with CNPC and newly diagnosed MDD using a large, geographically diverse dataset of commercially insured adults.

b) Use of a comprehensive and nuanced claims-based algorithm to identify adults with TRD in a unique population of adults with CNPC and MDD: The current study applied a comprehensive claims-based algorithm, developed using the Massachusetts General Hospital (MGH) clinical staging method. ${ }^{23,61}$ This algorithm has been previously used by Gibson et al. to identify adults with TRD and estimate the cost burden of TRD among adults with MDD. ${ }^{23}$ Several existing studies have defined TRD based on a single criterion or a combination of criteria such as number of antidepressant switches, number of titrations, and concomitant administration of antidepressants, mood stabilizers or atypical antipsychotics. ${ }^{22,29,30,46}$ Unlike approaches based on a single criterion, the MGH scoring based approach used in this study is comprehensive as it includes the four main strategies to overcome lack of response i.e. optimization, switching, combination or augmentation. ${ }^{62}$ Further this approach can be easily translated to prescription data available in pharmacy claims. ${ }^{23}$ To date, no study has used this approach to identify TRD among adults with CNPC and MDD. 
c) Novel risk factors of TRD: As the current study specifically focuses on adults with MDD and CNPC, it used several novel risk factors of TRD such as polypharmacy, type of pain treatment, and number of CNPC conditions. Association of these risk factors with TRD has not been established in previous studies on adults with MDD.

d) Fill a significant knowledge gap in literature: Given that no studies have examined TRD among adults with co-existing CNPC and MDD, the proposed study will fill an important knowledge gap in literature by identifying leading predictors of TRD, quantifying excess economic burden associated with TRD and examining the association between TRD and LTOT.

e) Use of advanced predictive modeling to identify leading predictors of TRD using routinely collected insurance claims data: use of predictive modeling to identify patients who are at risk for transitioning from MDD to TRD among individuals with CNPC will help in understanding modifiable factors which can guide clinicians in intervening early to reduce the risk of TRD. Additionally, targeted interventions can be designed for this sub-group of patients to improve clinical outcomes and reduce future medical costs and, healthcare utilization.

f) Application of econometric techniques to identify the difference in characteristics between patients with and without TRD that explain the excess total healthcare expenditures associated with TRD: This is the first study to examine the contribution of various patient level differences between adults with TRD and without TRD to the observed gap in total healthcare costs between the two groups from a population based perspective.

\subsection{Specific Aims}


AIM 1: Identify leading predictors of transitioning from MDD to TRD among adults with

CNPC and newly diagnosed MDD in a real-world setting using a machine learning approach.

Hypothesis: In adjusted models, that include all the predictors, presence of chronic conditions, anxiety disorders, sleep disorders, substance- use disorders, age, and polypharmacy will be leading predictors of TRD among adults with CNPC and newly diagnosed MDD.

AIM 2: Estimate the direct economic burden (direct healthcare costs and utilization) of TRD and its contributory factors among adults with CNPC and newly diagnosed MDD using post-regression decomposition techniques.

Hypothesis: Adults with TRD will have significantly higher direct healthcare costs and utilization as compared to adults without TRD; the majority of excess costs due to TRD will be explained by differences in the type of CNPC, presence of obesity, sleep disorders, and other chronic conditions between the two groups.

AIM 3: Examine the trajectory of LTOT over a 3-year period in adults with CNCP and the association of TRD with LTOT among adults with CNPC and newly diagnosed MDD.

Hypothesis: The use of LTOT will decline over the 3-year study period and adults with TRD will be more likely to use LTOT as compared to adults without TRD.

\subsection{Approach}

\section{Conceptual Framework}

Aim 1: Extensive literature review and a conceptual framework adapted from the modified determinants of health outcomes and chronic disease model was used to identify the plausible risk factors of TRD. ${ }^{63}$ This framework was originally proposed by Wilkinson and Marmot. ${ }^{63}$ 
The model suggests that incidence of a disease (in this case TRD) can be affected by several dimensions. These dimensions include: 1) community resources (e.g. geographical region), 2) access to care factors (e.g. Medicare enrollment, type of plan and co-pay), 3) treatment- related factors (e.g. pain treatment such as opioid use, NSAID use, use of psychotherapy, type of provider such as primary care provider, specialist, mental health specialist, polypharmacy, class of index antidepressant medication, and inpatient status), 4) biological risk factors (e.g. age, sex), and 5) health-related factors which comprises of two sub-domains, mainly chronic health conditions (e.g. CVD, hypertension, diabetes, asthma, COPD, anxiety disorders, dementia, sleep disorder, osteoporosis, and type of CNPC), and lifestyle factors (e.g. substance use, tobacco use, alcohol use and obesity).

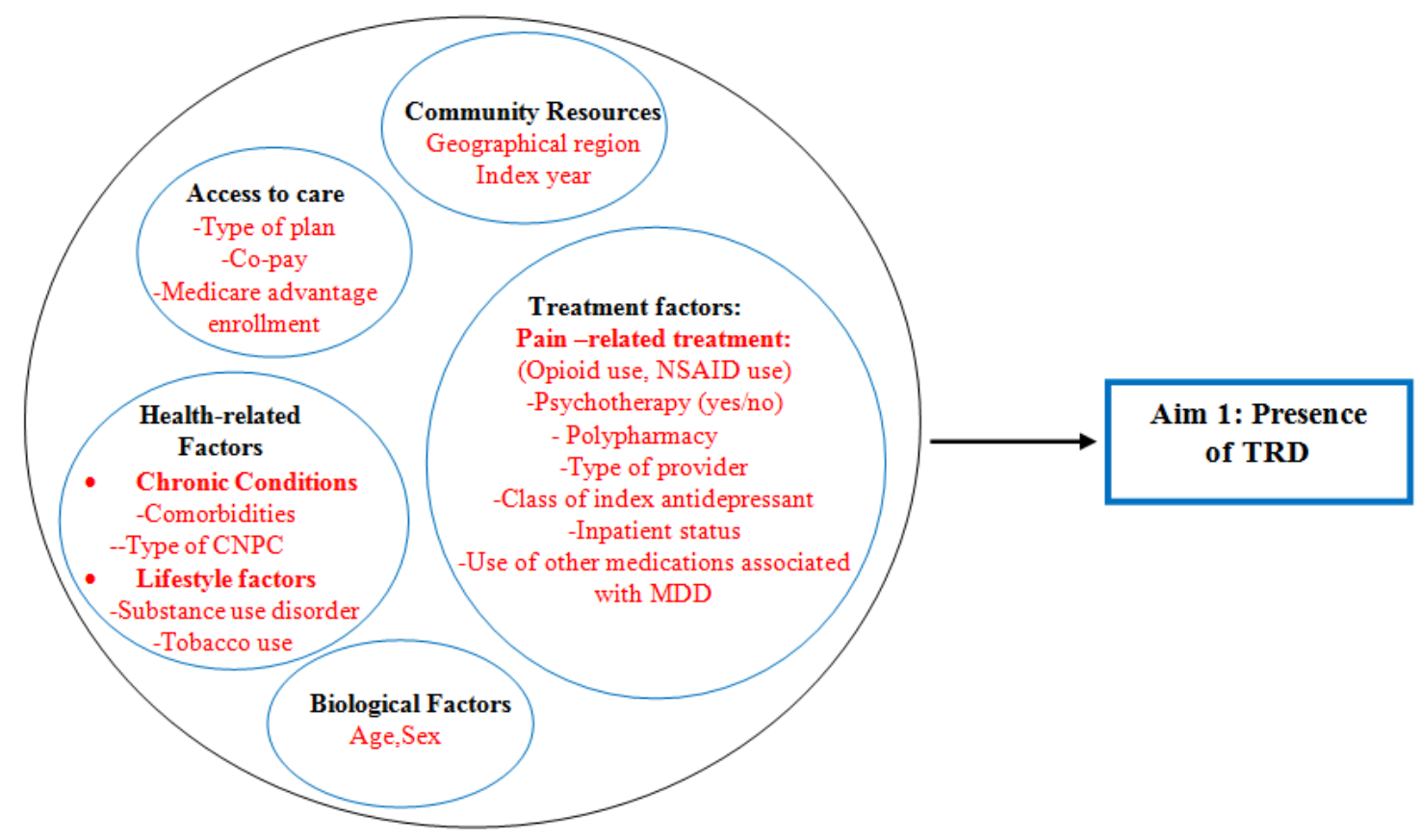

Figure 1.1: Adapted determinants of health outcomes and chronic disease model Aim 2: The conceptual framework for Aim 2 was based on the adapted Andersen's Behavioral Model of Health Services Use, ${ }^{64}$ which posits that healthcare utilization of an individual is a function of multiple factors. These factors can be grouped into four domains, 
including 1) predisposing factors that represent the individual characteristics predictive of using healthcare services (e.g., age, sex); 2) enabling factors that may enable access to healthcare services (e.g. Medicare advantage enrollment, type of insurance plan); 3) need factors that define an individual's health status (e.g. chronic conditions, obesity, sleep disorders, tobacco use disorder, and substance use disorder, polypharmacy, benzodiazepine use, and use of pain medication such as opioids and NSAIDs); and 4) environmental factors that may influence an individual's healthcare utilization (e.g. geographical region).

Aim 3: To guide the process of variable selection for aim 3 of the current study, the expanded Symptom Management Model was used. ${ }^{65,66}$ The generic symptom management model has three dimensions: symptom experience, management strategies and outcomes. The dimension of symptom experience can be described as perception of symptom as well as a response to a symptom. As per the proposed framework, presence of TRD, which is mainly nonresponse to depression treatment trials, was considered as a symptom which can influence the outcome of LTOT. As per the model, the above dimensions are influenced by the following three domains: (1) the personal domain, which comprised of demographic factors (e.g. age, sex, insurance plan type, Medicare advantage enrollment), psychological factors (e.g. presence of anxiety and other mental health comorbidities); (2) the health and illness domain (e.g. chronic physical conditions, obesity, sleep disorders, substance use disorders, polypharmacy, benzodiazepine use, and use of pain medication such as NSAIDs); (3) the environmental domain (e.g. geographical region, index year of MDD diagnosis). 


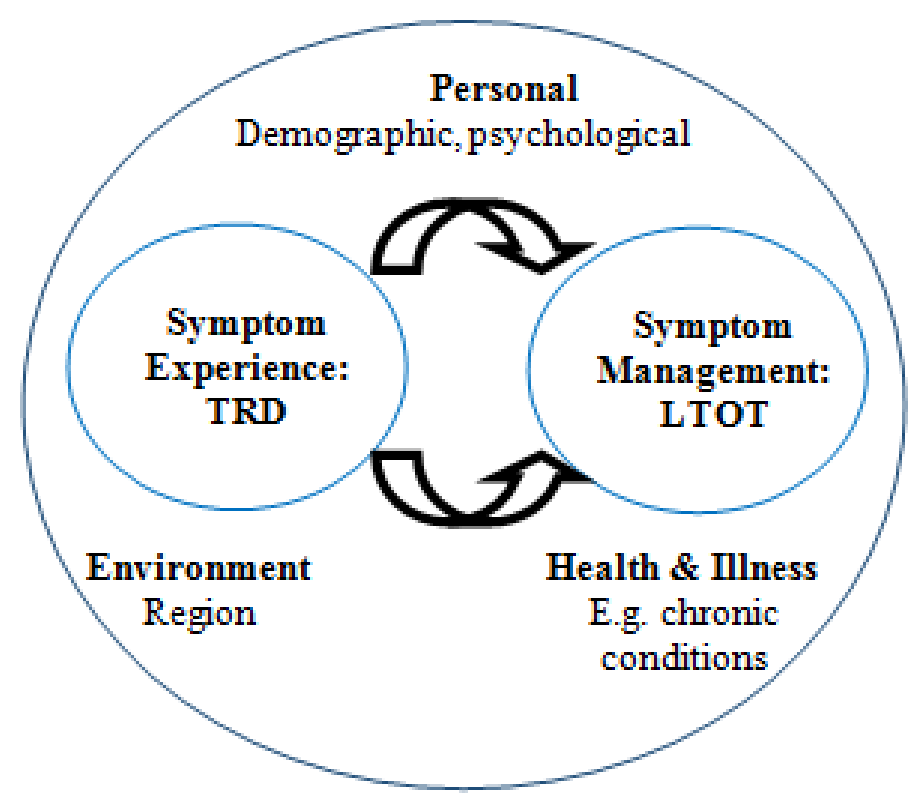

Figure 1.2 Expanded Symptom Management Model

\section{Data Sources}

To accomplish the study objectives, we used the $10 \%$ sample of de-identified health insurance claims data from Optum Clinformatics ${ }^{\circledR}$ DataMart (Optum Clinformatics ${ }^{\circledR}$, Eden Prairie, MN, USA) for the period of $1^{\text {st }}$ January 2007 through $30^{\text {th }}$ June 2017 . This nationally representative, geographically diverse database contains a combination of inpatient, outpatient and pharmacy claims for individuals enrolled in the commercial insurance plans and Medicare Advantage plan of a large U.S. health insurance company. The data also contains information on lab results, certain demographic characteristics (e.g year of birth, sex, residential state) and plan type (e.g. health maintenance organization, preferred provider organization, point of service, feefor-service )that are routinely collected during health insurance enrollment. ${ }^{67}$ The data contains a $10 \%$ random sample of information on 47 million individuals, approximately $80 \%$ of whom purchased insurance from their employers. 


\section{CHAPTER 2}

\section{Predictors of Treatment-Resistant Depression among Adults with Chronic Non-Cancer Pain Conditions and Major Depressive Disorder: A Machine Learning Approach}

\subsection{Abstract}

Objective: Presence of chronic non-cancer pain conditions (CNPC) among adults with major depressive disorder (MDD) often reduces benefits of antidepressant therapy, thereby increasing the possibility of treatment resistance. This study sought to identify leading predictors of treatment-resistant depression (TRD) among adults with newly diagnosed MDD and CNPC using machine learning approaches.

Methods: This retrospective cohort study included adults ( $\geq 18$ years) with newly diagnosed MDD and CNPC from a de-identified 10\% random sample of Optum ${ }^{\circledR}$ Clinformatics ${ }^{\circledR}$ Data Mart (2007-2017). TRD was identified using the Massachusetts General Hospital clinical staging algorithm for claims data. Random forest (RF), a machine learning method, was used to identify leading predictors of TRD. Initial model development included 42 known and/or probable risk factors for TRD. The final refined model included 20 risk factors. To facilitate comparison with published studies, results from logistic regression are presented.

Results: Included in the sample were 23,645 patients (73\% female mean age: 55 years; $78 \%$ with $\geq 2$ CNPC, and $91 \%$ with joint pain/arthritis). Overall, $11.4 \%(\mathrm{~N}=2,684)$ transitioned from newly diagnosed MDD to TRD. The five leading predictors of TRD were: mental health specialist visits, polypharmacy ( $\geq 5$ medications), psychotherapy use, anxiety, and age. Crossvalidated logistic regression model indicated that those with TRD were younger, more likely to have anxiety, mental health specialist visits, polypharmacy, and psychotherapy use with AORs ranging from 1.9-1.3 (all Ps $<0.01$ ). 
Conclusion: Machine learning identified several modifiable factors that warrant further investigation and may serve as potential targets for clinical intervention to improve treatment outcomes in those with TRD.

\subsection{Introduction}

Major Depressive Disorder (MDD) affects approximately 350 million adults worldwide and 35 million adults in the United States (US). ${ }^{3,68}$ Major Depressive Disorder is the leading cause of disability and a major contributor to the overall global illness burden. ${ }^{1,69}$ Despite the efficacy of various antidepressant medications in improving depression symptoms, many adults with MDD in real-world settings do not respond to repeated trials of antidepressants. ${ }^{70,71}$ Therefore, a significant proportion of the burden of MDD can be attributed to treatment resistant depression (TRD) ${ }^{20}$ Although there is lack of consensus on the definition of treatment resistant depression (TRD), the designation -"TRD" is primarily used to describe patients who do not respond to at least two adequate trials of antidepressants. ${ }^{17-20,72}$ Due to the variations in study population, follow-up time period, datasets used, and measures used to define TRD, the prevalence of TRD has ranged from $6 \%$ to $30 \%^{21}$ in published studies. For example, the Sequenced Treatment Alternatives to Relieve Depression $\left(\mathrm{STAR}^{*} \mathrm{D}\right)$ trial, the largest and longest study ever conducted to evaluate the effectiveness of depression treatment reported that even after undergoing four sequential antidepressant trials, $30 \%$ of patients did not achieve remission. $^{21,72}$

Published studies have suggested that some subgroups of patients with MDD may be more likely to have TRD. ${ }^{20,22,24,29}$ These studies have consistently reported higher prevalence of TRD among adults with chronic non-cancer pain conditions (CNPC). ${ }^{20,22,24,29}$ It is plausible that individuals with CNPC may be more likely to transition into TRD because MDD and its 
treatment can be affected by pain, often considered as a somatic symptom of depression. ${ }^{13} \mathrm{~A}$ growing body of literature has documented the bi-directional relationship between pain and MDD. As pain and MDD are inextricably linked in terms of the shared biological pathways and neurotransmitters, they often respond to similar treatments, and may also exacerbate one another. Some researchers have labeled this relationship as depression-pain syndrome. ${ }^{14,15}$ A narrative review on MDD and pain estimated that the prevalence of MDD in patients with CNPC can be as high as $85 \%$ and can range from $32 \%-85 \%$ depending on the type of condition and setting. ${ }^{7} \mathrm{~A}$ study by Biar et al. concluded that presence of baseline CNPC among adults with MDD reduces the benefits of antidepressant therapy at 12 weeks, suggesting treatment resistance. ${ }^{11}$ Indirect evidence of the relationship between CNPC and TRD has been reported. A Canadian Study by Rizvi et al. and a US study by Kubitz et al. reported that the prevalence of CNPC such as joint pain, muscle pain, headache/migraine, and back pain was two times higher among patients with TRD, as compared to those without TRD..$^{22,24}$

The transition from MDD to TRD can be influenced by many factors. In studies not specific to CNPC, severity of depression, response to first antidepressant treatment, number of past depressive episodes, obesity, highly activated inflammatory system, inpatient status, presence of anxiety, insomnia, pain, and other social factors have been identified as risk factors of TRD ${ }^{20,22,26,28,29,50,73,74}$ While there is literature on risk factors of TRD among general population with MDD, among a sub-group of adults with CNPC and MDD, additional probable risk factors such as pain therapy commonly used for CNPC needs to be examined. Certain commonly used analgesic medications and other medications such as benzodiazepines, corticosteroids, and beta-blockers may have mood related adverse effect or have higher rates of drug-drug interactions with antidepressants which can lead to safety concerns, poor clinical 
response, and may increase the risk of TRD. ${ }^{36,37}$ As adults with CNPC are more likely to have polypharmacy, the potential for drug-drug interactions may be greater with severe health consequences. $^{75,76}$ Therefore, polypharmacy as a predictor of TRD needs to be investigated.

To our knowledge, to date, no study has systematically examined the risk of TRD among adults with CNPC and MDD. In this context, the first critical step in filling this knowledge gap is an examination of risk factors that can influence the transition from MDD to TRD among adults with CNPC. When a patient is newly diagnosed with MDD, the presence of characteristics that could increase the risk of TRD may alert healthcare providers to monitor the patients more closely. Identification of leading predictors of TRD in real-world clinical settings with robust statistical and machine leaning methods can help clinicians understand treatment response and inform targeted individualized treatment approaches. Furthermore, non-parametric machine learning methods can improve predictive accuracy by reducing over fitting even in the presence of complex interactions, as compared to standard parametric approaches. ${ }^{77}$ As these predictive models require a large of number of observations and predictors, population-based claims data

can be ideal. ${ }^{38,39}$ Therefore, the primary objective of the study is to identify leading predictors of transitioning from MDD to TRD among adults with CNPC and newly diagnosed MDD in a realworld setting using a machine learning approach.

\subsection{Methods}

\section{Study design and Data Source}

We used a retrospective cohort study design. The cohort included all adults (age $\geq 18$ years) with newly diagnosed MDD and CNPC. We used de-identified patient data with medical and pharmacy claims as well as enrollment information from the Optum® Clinformatics® Data Mart $^{67}$ for the period January 2007 to June 2017. For this study, we used a $10 \%$ random sample 
extracted from a nationally representative, geographically diverse database of 47 million individuals. The data comprised adults who were insured in a commercial plan as well as Medicare Advantage plans. ${ }^{67}$

Newly diagnosed MDD was identified between January 2008 and June 2016 using a validated and published algorithm. ${ }^{78,79}$ The first observed MDD diagnosis during a calendar year was identified using ICD-9-CM (International Classification of Diseases, Ninth Revision, Clinical Modification) or ICD-10 CM (International Classification of Diseases, Tenth Revision, Clinical Modification) diagnosis codes as appropriate (See in Appendix 7.1). A 180-day lookback period was used to ensure that the individuals did not have any MDD claims or antidepressant prescriptions.

The date of newly diagnosed MDD (index date) was used to create the baseline period (12 months before the index date) and the follow-up period (12 months after the index date). The baseline period was used to identify CNPC and other predictors (see Measures section). The follow-up period was used to identify TRD and MDD treatment-related factors during the acute phase of treatment, before TRD identification (first 90 days after MDD diagnosis) (Appendix 7.2).

\section{Analytical Sample}

The analytical sample consisted of adults with CNPC identified in the 12 months before MDD diagnosis. CNPC was identified using an extensive list of 1000 conditions for which pain was severe enough to call them as CNPC. ${ }^{80-82}$ The diagnoses were further collapsed into four major pain categories: arthritis/joint pain, back/neck pain, headache/migraine, and neuropathic pain/other chronic pain conditions. ${ }^{80-82}$ We required at least two healthcare encounters in an inpatient or outpatient setting for identification of adults with CNPC. The ICD 9/10 diagnosis 
codes used to identify CNPC are provided in the Appendix 7.2. Other inclusion/exclusion criteria were: at least one antidepressant prescription after MDD diagnosis; continuous enrollment during the baseline and follow-up period (24 months); no cancer diagnosis at any time during the study period; and consistent with prior studies, individuals having any diagnosis of schizophrenia, schizoaffective disorder, other psychosis, manic depression or bipolar disorders

were excluded. ${ }^{22,23}$ The final analytical sample consisted of 23,645 adults with CNPC and newly diagnosed MDD (See Figure 1 for number of individuals eliminated at each step of inclusion/exclusion criteria).

\section{Outcome}

\section{Treatment-Resistant Depression}

The primary outcome was the transition to TRD within a year after the index date. Identification of TRD using insurance claims data is challenging for several reasons: 1) there is no universally accepted definition of TRD; 2) lack of information on depression symptoms and severity; and 3) availability of prescription rather than actual use of antidepressants. ${ }^{42}$ Therefore, studies using administrative claims have used several different algorithms to define TRD. These have typically included single criterion such as number of antidepressant switches, number of titrations, concomitant administration of antidepressants, mood stabilizers or atypical antipsychotics, or a scoring method based on all of the above. ${ }^{22,23,41,42}$ Additionally, use of electroconvulsive therapy is also considered as a marker of TRD because ECT is often used as a late-stage treatment for TRD. ${ }^{41}$

We applied the Massachusetts General Hospital (MGH) clinical staging method to insurance claims to identify individuals with TRD. This algorithm has been previously used to predict the cost burden of TRD ${ }^{23,61}$ We selected this algorithm because it includes the four main strategies 
to overcome lack of therapeutic response, i.e. optimization, switching, combination or augmentation and can be easily translated to prescription data available in pharmacy claims. ${ }^{62}$ Using this approach, we assigned 1 point for each adequate antidepressant trial (i.e., two or more fills of the same antidepressant), half a point for each of the following optimization strategy: extended duration defined as $\geq 3$ fills of the same antidepressant, an upward titration in dose, and augmentation with atypical antipsychotic/mood stabilizer. An overall score exceeding three was used as a threshold to establish TRD and those with a score exceeding three were classified as having TRD. ${ }^{23} \mathrm{An} \mathrm{MGH}$ score of three is the equivalent to two distinct antidepressant trials with one optimization strategy such as augmentation. ${ }^{29}$ Any additional optimization or augmentation strategy would meet the threshold for TRD. ${ }^{83}$ Those using electroconvulsive therapy (ECT) were considered to have TRD.

\section{Statistical and Prediction Analyses}

Unadjusted associations between predictors and TRD were examined using chi-square tests for categorical variables and t-tests for continuous varibles. Multivariable logistic regression (LR), a method familiar to many researchers is a standard technique for analysis of binary outcomes in medicine and social science, where the focus is not only on prediction, but also on explaining the association between variables. ${ }^{84}$ Logistic regression, a standard parametric method makes several assumptions about the data. ${ }^{84}$ Recently, algorithmic or machine learning methods that do not have any strong parametric assumptions have gained popularity because they can be used on large complex datasets and can provide better accuracy ${ }^{85} \mathrm{~A}$ common theme among all the algorithmic models is validation and testing of models using random bootstrap samples. Although many types of machine learning methods are available for binary outcomes such as the presence or absence of TRD, random forest $(\mathrm{RF})$ is a particulary attractive alternative because of 
its many advantages such as high predictive accuracy, reduction in overfitting, and identification of most useful set of features. RF is based on decision trees, which represent the relationships of variables in the dataset. Each decision tree models hierarchical relationships between predictors and outcomes by maximing the amount of information gain.

We used the caret and randomForest package for $\mathrm{R}$ software for building the machine learning predictive models using LR and RF. ${ }^{86}$ To create and train the models the data was randomly split into a $70 \%$ training sample $(\mathrm{N}=16,570)$ and was internally validated using a $30 \%$ test sample $(\mathrm{N}=7,075)$. The training sample was used for model refinement, learning, and to determine the model coefficients, while the test sample was used to evaluate the model performance on a previously unseen data.

RF requires input of the number of decision trees to grow for each run and the number of features to be used for each tree. After model training and tuning, the number of trees for each run was determined to be 100 and the number of features was set to five. The trained fully adjusted model and the model with top 20 predictors were then tried in the test data and prediction accuracy was computed for the treatment outcome. The area under the curve (AUC) was calculated to assess the performance of the predicting models and measures of discrimination, including sensitivity and specificity are reported. To facilitate model interpretation, the adjusted odds ratio and their $95 \%$ confidence intervals computed from performing logistic regression of the final model are presented for the top predictors identified from the machine learning method. Dataset construction was performed using SAS 9.4 (Cary, NC) and all predictive modeling was performed in R software (R Development Core Team, Vienna, Austria).

\section{Predictors (i.e. Features) of TRD for Inclusion in the Model}


While feature selection can be performed using automated methods, many experts suggest that variable selection should be supported by expert knowledge or conceptual frameworks, whenever feasible. ${ }^{87}$ Initial selection of the plausible predictors of TRD $(n=42)$ was based on the Determinants of Health Outcomes and Chronic Disease conceptual framework, ${ }^{63}$ that included the following dimensions: 1) biological risk factors, 2) access to care factors (e.g. type of plan and co-pay), 3) disease and health- related factors 4) treatment-related factors (MDD-related treatment, pain-related treatment, and other treatment factors), and 5) community resources. For a comprehensive list of all predictors see Table 2.1. While most variables were measured during the baseline period, MDD-treatment related variables were measured during the follow-up period, before TRD measurement. In order to ensure that all included variables were at least plausible predictors of TRD, a manual variable selection was performed using an extensive literature review and a conceptual framework adapted from the modified Determinants of Health Outcomes and Chronic Disease conceptual framework. ${ }^{63}$

Biological factors included age and sex. Predictors included in access to care domain were the type of plan, and out of pocket payment (calculated using co-pay, co-insurance and deductible for healthcare services). Disease-related factors included type and number of CNPC and health-related factors included other chronic conditions, obesity, substance use disorders (including tobacco use disorder, alcohol use disorder, and drug use disorders). All health-related factors were measured using ICD-9 or ICD-10-CM codes as appropriate. Pain-related treatment factors included commonly used pain medications such as opioid use, and NSAID use. MDDrelated treatment factors included mental health specialist visits, inpatient status, use of psychotherapy after MDD diagnosis, and class of index antidepressant medication. Other treatment-related factors included primary care provider visits, polypharmacy (defined as 
concomitant use of $\geq 5$ medications within 90 days $)^{88}$, and use of medications that may have depression as a side-effect (included fills for benzodiazepines, analgesics such as NSAIDs and opioids, statins, corticosteroids, and beta blockers). ${ }^{36}$

\section{Model Development}

To identify an abbreviated set of factors that can increase the utility of the predictive model for providers and other stakeholders, a more refined model was built using basic filter method such as chi-square for categorical variables and pearson's correlation for continuous variables. Further, an alternative wrapper feature selection method using logistic regression was used, wherein the performance of regression models with subsets of variables is assessed in random subsets of data. ${ }^{89}$ Feature selection yielded a total of 20 variables for inclusion in the refined model. Figure in the Appendix 7.3 illustrates the process of variable selection and model development.

\subsection{Results}

Characteristics of the study sample (total $\mathrm{N}=23,645$ ) are summarized in Table 2.2. Majority were females (73.3\%) and the mean age of patients was 55.36 years. Arthritis/joint pain was the most commonly observed pain condition (91\%) followed by back/neck pain (71\%). A large proportion of study sample reported $\geq 2 \mathrm{CNPC}$ conditions $(78 \%)$. The most commonly observed other chronic conditions were cardiovascular conditions (38.4\%) and anxiety (27.6\%). About one forth of the study subjects used pain medications such as opioids (28.7\%) and NSAIDs $(25.5 \%)$.

In our study sample, 2,684 patients $(11.4 \%)$ had TRD within one year after MDD diagnosis. About $3.3 \%$ of the study sample augmented antidepressants with an atypical 
antipsychotic or a mood stablizer and only 11 patients were found to be using electroconvulsive therapy within one year of MDD diagnosis. (Supplementary Information, Appendix 7.4)

There were statistically significant differences in TRD status (Table 2.2) by age, type and number of CNPC, other chronic conditions such as anxiety, presence of sleep disorders, substance use disorders, polypharmacy, and use of MDD-related treatment factors (All $p<$ 0.001). For example, the prevalence of TRD was significantly higher among younger adults (3544 years old) as compared to those with age $\geq 65$ years $(13.0 \%$ vs. $9.2 \%)$, among those with anxiety $(15.3 \%$ vs. $9.9 \%)$, with $\geq 3$ CNPC conditions (14.1\% vs. $9 \%)$ as compared to those with one CNPC. A significantly higher proportion of adults with TRD had drug use disorder (19.8\% vs. $11.0 \%)$, used polypharmacy (14.9\% vs. $8.5 \%)$, and benzodiazepines in the baseline period (15.4\% vs. 9.8\%). Additionally, greater proportions of adults having inpatient hospitalizations, and mental health specialist visits within 90 days of MDD diagnosis had TRD as compared to those having no inpatient or mental health specialist visits $(p<0.001)$. Adults who were prescribed SSRI as the first antidepressant after MDD diagnosis had lower prevalence of TRD, whereas those whose index prescription belonged to the class of other antidepressants and TCAs had higher prevalence of TRD $(p<0.001)$.

The fully-adjusted predictive model as well as the reduced predictive model with top 20 features had fairly modest discriminatory model performance. The AUC obtained from the RF model for the training data was 0.716 and 0.70 for the fully-adjusted and refined models respectively. The out of bag (OOB) error, which is an indicator of the unbiased estimator of true error rate, ranged from $11.4 \%-11.5 \%$. Similar model performance was observed in the test data (Supplemental information, Appendix 7.5). The AUC for the RF and LR models were similar. The AUC from the training sample for the fully adjusted LR model was 0.711 for the fully- 
adjusted model and 0.704 for the refined model and from the test sample for the fully adjusted and refined models was 0.708 and 0.705 respectively.

The average importance values obtained from LR and RF for the highest scoring top 10 predictors of TRD are represented in Figure 2. Overall, mental health specialist visits, polypharmacy, psychotherapy use, presence of anxiety, younger age, index antidepressant class, sex, any IP hospitalization after MDD diagnosis, number of CNPC, and benzodiazepine use were the leading predictors of TRD in both RF and LR, although ordering were slightly different.

For ease of interpretation and for comparison with published literature, Table 2.3 summarizes the adjusted odds ratios (AOR) and their associated 95\% CIs obtained from logistic regression of the final refined model (model with top 20 features). For example, adults visiting mental health specialists within 90 days of MDD diagnosis were almost two times more likely to develop TRD, as compared to those not having mental health specialist visits ( $\mathrm{AOR}=1.93 ; 95 \%$ $\mathrm{CI}=1.7-2.18)$. Adults using psychotherapy after MDD diagnosis $(\mathrm{AOR}=1.36,95 \% \mathrm{CI}=1.20$ 1.55 ) and those using $\geq 5$ different types of medications concomitantly in a period of 90 days ( $\mathrm{AOR}=1.81,95 \% \mathrm{CI}=1.62-2.03)$ were more likely to develop TRD, as compared to their counterparts without these characteristics.

\subsection{Discussion}

To our knowledge, this is the first study using large administrative claims data to identify leading predictors of TRD among commercially insured adults CNPC and newly diagnosed MDD. In the current study, approximately one in nine adults (11.4\%) with CNPC and newly diagnosed MDD transitioned to TRD within 1 year. This estimate is consistent with previously reported estimates for TRD prevalence among adults with MDD which ranged from 6\% to $30 \% .^{22,23,29,44}$ It has to be noted that TRD rates from our study are not directly comparable to 
published studies due to differences in study population as our study sample was restricted to adults with CNPC and newly diagnosed MDD. Our estimate of $11.4 \%$ for TRD prevalence, which was identified using a clinical staging claims algorithm, ${ }^{23,61}$ was in line with a recent study that reported the prevalence rate of TRD as $11 \%$ using the Optum database. It has to be noted that the previous study used a slightly different definition for identifying TRD subjects as those with at least three distinct trials of antidepressants or those who augmented antidepressants with antipsychotics. ${ }^{29}$

Consistent with published literature we found age to be a leading predictor of TRD. ${ }^{29,30}$ It has been reported in literature that TRD has been linked to an earlier age of onset of depression, ${ }^{90,91}$ and our study findings further support this. Sex has not been previously reported as a leading predictor of TRD. ${ }^{26,27,29,50}$ However, the relevance of sex in this patient population is not new..$^{22,51,55}$ This finding could perhaps be attributed to the higher prevalence and risk of both MDD as well as chronic pain among females, as compared to males. ${ }^{22,30,92}$ It has to be noted that our study sample was overwhelmingly female (73\%), which is comparable to the $61 \%-71 \%$ prevalence estimates of females in studies conducted among all adults with MDD. ${ }^{22,29,41}$

Our findings demonstrated that in clinical settings, a smaller set of MDD treatmentrelated factors around the time of MDD diagnosis can be used to predict the risk of transitioning from MDD to TRD. These included the class of first prescribed antidepressant after MDD diagnosis, mental health specialist visits, and psychotherapy use within the first 90 days after a MDD diagnosis. Specifically, we found that adults with CNPC who used SSRIs, the most frequently prescribed antidepressants, ${ }^{93}$ as their index antidepressant were less likely to transition from MDD to TRD. Existing studies on association between the specific type of antidepressants and outcomes in patients with TRD had a very small sample size and resulting conclusions 
should be taken cautiously. ${ }^{74}$ In light of the findings from the current study, future research is warranted to understand the association between types of antidepressants with TRD. The predictors such as mental health specialist visits and psychotherapy use are often indicative of greater depression severity. ${ }^{94}$ As patients with more severe MDD or patients with poor response to antidepressants may have a higher chance of visiting a mental health specialist or using psychotherapy as additional treatment, ${ }^{94}$ they may have a higher risk of TRD.

The current study identified anxiety as well as benzodiazepine to be predictors of TRD. While, comorbid anxiety disorder has been consistently reported by several studies as a risk factor for TRD, ${ }^{26,74,90,91}$ association of benzodiazepine use with TRD has not been investigated in prior literature. Treatment becomes challenging when pain, often experienced by those with CNPC and MDD, overlap with anxiety. Researchers have highlighted the reciprocal interactive relationships between pain, anxiety and depression. ${ }^{51,91}$ It is plausible that anxiety is a risk factor for TRD because comorbid anxiety disorder and MDD in patients with CNPC is often associated with higher symptom severity and poor response to depression treatment. ${ }^{51,91}$ Benzodiazepines are commonly used medications for anxiety and sleep disorders, both common comorbid conditions among patients with CNPC and MDD. In fact, studies have shown that the use of benzodiazepines is significantly higher among patients with chronic pain. ${ }^{95,96}$ Previous research has documented a high prevalence of benzodiazepine use among women, especially older women, and among opioid users. ${ }^{97,98}$ Despite several major medical and psychiatric organizations advising against using benzodiazepines among elderly and in combination with opioids, benzodiazepines continue to be prescribed. This may lead to high risks and serious adverse effects among vulnerable groups. ${ }^{99,}{ }^{96}$ Benzodiazepine use as a predictor of TRD further reinforces the need to weigh the risk versus benefits of prescribing benzodiazepines among 
adults with CNPC and newly diagnosed MDD. Our findings suggest that patients with CNPC, MDD and anxiety disorders need to be monitored more closely and that there is a need for evaluating alternative treatment strategies that can help in the co-management of these conditions.

We also identified the number of CNPC conditions as an important predictor of TRD. Higher number of CNPC conditions may indicate multisite chronic pain and may result in increased pain severity which is a strong predictor of poor depression outcomes. ${ }^{11}$ Studies have also reported that chronic pain was associated with increased severity of depression and insufficient response to antidepressants. ${ }^{11,100}$ Several large clinical trials on chronic pain often exclude patients with MDD, ${ }^{101}$ and therefore lack of data on treatments that can be used to comanage both pain and MDD continues. Our findings underscore the need for effective pharmacological treatments for managing both pain and MDD symptoms.

Our predictive models identified polypharmacy (excluding the use of antidepressants) as one of the top five predictors of TRD. Pain medications are commonly used to relieve pain among patients with CNPC. While we did not find individual pain medications such as NSAIDs and opioids as risk factors for TRD, a combination of medications may increase the potential exposure to side-effects and harm due to drug-drug interactions. In fact, a recent study which characterized polypharmacy among patients with CNPC suggested that a majority of medication related-harm exposure was also attributed to medications other than opioids, such as simple analgesics, benzodiazepines, and number of medications used concomitantly. ${ }^{75}$ Higher number of concomitant medication use is also indicative of the presence of multiple chronic conditions which are more common in patients with TRD, as compared to those without TRD. ${ }^{20}$ 
Although machine learning methods and standard logistic regression method yielded similar model performance, adoption of RF and LR machine learning approaches enabled us to perform robust feature selection and in identifying the most important predictors out of an extensive set of 40 features. The model performance of fully adjusted as well as with a reduced set of 20 variables was comparable and fairly modest. A reduced set of top $n$ features $(n=20)$ helps in focusing on the most important predictors of TRD and is more practical if one was to design a new study and collect features to predict TRD among CNPC patients.

\subsection{Limitation}

Although a rigorous methodology was utilized to design and execute the study, the findings of this study need to be interpreted in the context of its limitations. The study suffers from inherent limitations associated with claims data analysis, including coding inaccuracies, absence of information on severity of MDD, socioeconomic status, race/ethnicity, physical activity, and social support. Furthermore, our algorithm used to identify MDD naïve patients (a look-back period of 180 days) may not truly capture all incident MDD patients. For patients having multiple MDD episodes in the study period, we selected the very first episode, however, it is possible that they could have received diagnosis or treatment elsewhere, which could not have been captured in the current claims. It has to be noted that lack of availability of severity of MDD and pain may have resulted in unobserved selection bias. Further, we may not have truly captured the actual use of prescription medication as the drug claims provide information on only filled prescriptions and not actual use.

Although we identified leading predictors of TRD in a sample of CNPC patients with newly diagnosed depression, the model had a much better discriminative power to predict nonTRD cases as compared to TRD cases. One plausible explanation is that published prediction 
models with better sensitivity to identify TRD cases used data from participating clinical centers across Europe or clinical trial data which predicted TRD among prevalent cases of MDD. This can provide information on the number of past depressive episodes, severity of MDD, and other

clinical parameters which could not be captured via administrative claims data. ${ }^{26-28,73}$ Additionally, in actual clinical practice, adults with TRD are identifying using detailed clinical criteria such as responsiveness to medical treatments, and tolerance to antidepressants, which were not available in claims data. Therefore, we may have either underestimated or overestimated the prevalence of TRD. The models were refined and tested in unique sub-sample that was created from the same overall sample as the training data. The validity of the model and the leading predictors obtained from the models will be more generalizable if applied to datasets with patients with CNPC obtained from different samples, specifically other commercial administrative databases.

\subsection{Conclusion}

Notwithstanding the limitations, our study provides important insights on factors that may predict the transition from MDD to TRD among patients with CNPC using a large population-based real-world study. We identified biological and health-related factors unique to CNPC who developed MDD such as number of CNPC conditions, age, sex, other chronic conditions such as anxiety, polypharmacy and factors related to MDD treatment and severity such as mental health specialist visits, use of psychotherapy, and class of index antidepressant as leading predictors of TRD. These factors can serve as targets for future studies to further understand TRD and can help in developing healthcare and clinical interventions to improve treatment outcomes in patients with CNPC and MDD. 
Figure 2.1 Schematic presentation of study sample selection-Inclusion and exclusion criteria applied to patients with CNPC and newly diagnosed episode of MDD, Optum Clinformatics Data Mart, observation period January 2007-June 2017.

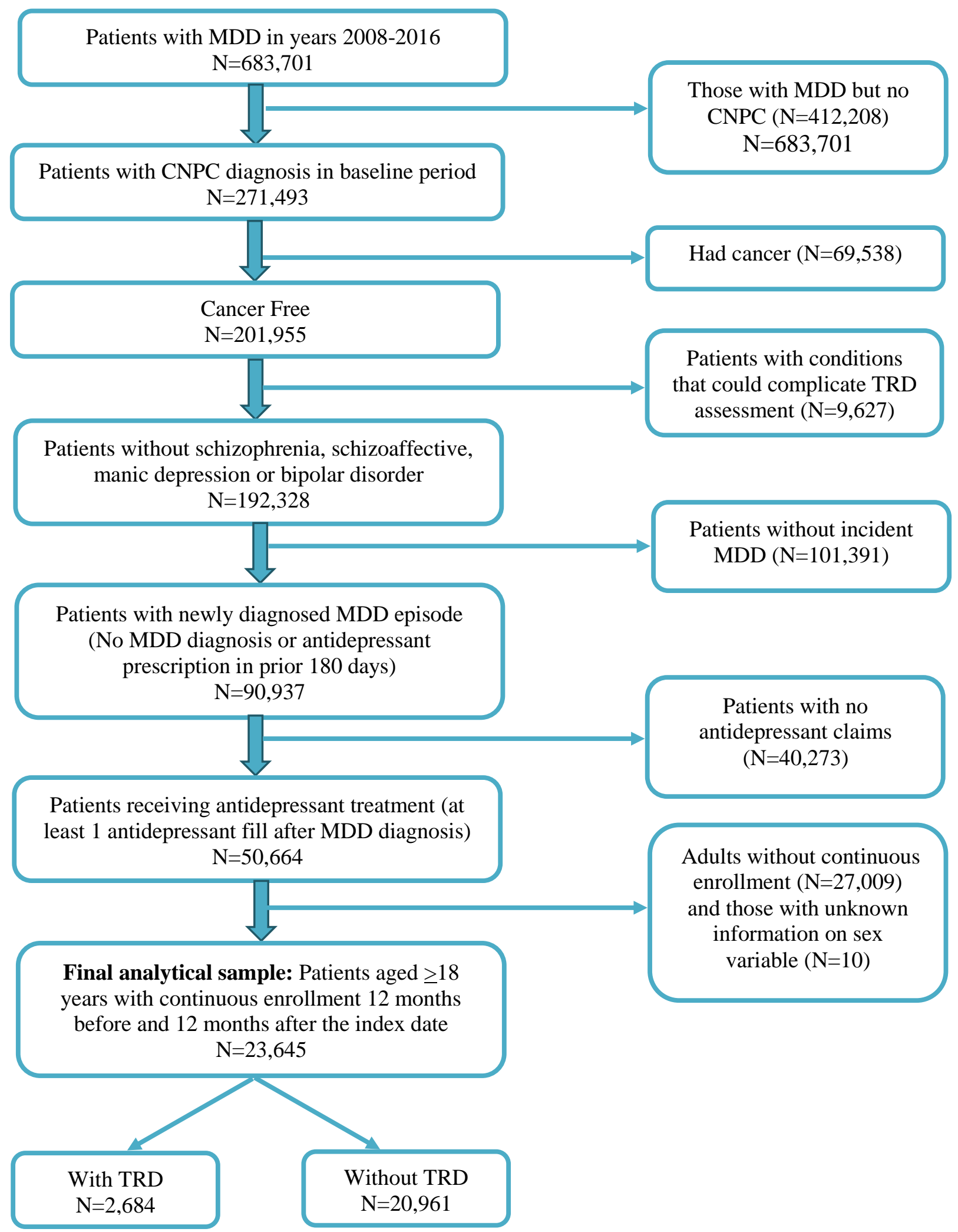




\begin{tabular}{|c|c|}
\hline \multicolumn{2}{|c|}{$\begin{array}{l}\text { Table 2.1. List of All 42 Predictors Considered for Model Inclusion Described by the Conceptual } \\
\text { Framework Dimensions }\end{array}$} \\
\hline Dimension & Predictors \\
\hline Biological risk factors $(\mathrm{N}=2)$ & Age, sex \\
\hline Access to care factors $(\mathrm{N}=3)$ & $\begin{array}{l}\text { Medicare enrollment, (commercial, Medicare advantage), plan type }{ }^{\text {a }}(\mathrm{HMO}, \\
\text { PPO/POS, other), out of pocket payment }\end{array}$ \\
\hline $\begin{array}{l}\text { Disease and Health-related } \\
\text { factors }(\mathrm{N}=18)\end{array}$ & $\begin{array}{l}\text { Type of CNPC (grouped into commonly occurring CNPC conditions including } \\
\text { back/neck pain, arthritis/joint pain, headache/migraine, neuropathic/other } \\
\text { chronic pain), number of CNPC conditions, chronic conditions including } \\
\text { anxiety, respiratory conditions (asthma and COPD), hypertension, } \\
\text { cardiovascular conditions }{ }^{\text {b }} \text {, dementia, diabetes, other mental illness, } \\
\text { osteoporosis, sleep disorder, obesity diagnosis, tobacco use disorder, alcohol } \\
\text { use disorder, drug use disorder }\end{array}$ \\
\hline $\begin{array}{l}\text { Treatment-related factors } \\
\text { (MDD -related, pain- related } \\
\text { and other treatment factors) } \\
(\mathrm{N}=17)\end{array}$ & $\begin{array}{l}\text { Pain - related treatment (included NSAID use, opioid use), use of } \\
\text { psychotherapy before MDD diagnosis, polypharmacy }{ }^{c} \text {, use of medications } \\
\text { associated with depression including benzodiazepines, corticosteroids, beta } \\
\text { blockers, and statins, primary care provider visits, mental health specialist } \\
\text { visits, use of psychotherapy after MDD diagnosis, any inpatient } \\
\text { hospitalizations, hospitalizations related to MDD, class of index antidepressant } \\
\text { (SSRI, SNRI, TCA, Other) }^{\mathrm{d}}\end{array}$ \\
\hline Community resources $(\mathrm{N}=1)$ & geographical region (region derived from state variable) \\
\hline Other predictors $(\mathrm{N}=1)$ & index year \\
\hline $\begin{array}{l}\text { a HMO: Health Maintenance Or } \\
\text { type includes fee-for-service, in } \\
\text { organization), IPP (individual pr } \\
\text { Non-steroidal anti-inflammatory } \\
{ }^{b} \text { Cardiovascular conditions incl } \\
\text { heart failure, myocardial infarcti } \\
{ }^{c} \text { Polypharmacy was defined as c } \\
{ }^{d} \text { SSRI: Selective serotonin reupt } \\
\text { antidepressants, other: includes } 1 \\
\text { vortioxetine. } \\
\text { Note: MAOI (monoamine oxida } \\
\text { (N=4) } \\
\text { Note: Predictors related to MDD } \\
\text { specialist visits, hospitalizations } \\
\text { diagnosis. }\end{array}$ & $\begin{array}{l}\text { zation, PPO: Preferred provider organization, POS: Point of service, other plan } \\
\text { nity plans, EPO (exclusive provider organization), GPO (Group purchasing } \\
\text { am plan), and other plans, CNPC: Chronic non-cancer pain conditions, NSAID: } \\
\text { gs } \\
\text { l hyperlipidemia, ischemic heart disease, coronary artery disease, congestive } \\
\text { and stroke } \\
\text { omitant use of } 5 \text { or more medications within a 90-day period } \\
\text { inhibitors, SNRI: Serotonin norepinephrine reuptake inhibitors, TCA: tricyclic } \\
\text { ications such as bupropion, mirtazapine, nefazodone, trazadone, vilazodone, and } \\
\text { hibitors) are not included as a predictor because of very small sample size } \\
\text { atment such as use of psychotherapy, index antidepressant, mental health } \\
\text { e measured in acute phase of MDD treatment, i.e. first } 90 \text { days after MDD }\end{array}$ \\
\hline
\end{tabular}




\begin{tabular}{|c|c|c|c|c|c|c|c|c|c|c|c|c|c|c|c|}
\hline \multicolumn{16}{|c|}{$\begin{array}{l}\text { Table 2.2. Description of Adults with CNPC and Newly Diagnosed MDD by TRD } \\
\text { Optum Clinformatics Real-World De-identified Data, 2007-2017 }\end{array}$} \\
\hline \multirow[b]{2}{*}{ Characteristics } & \multicolumn{2}{|c|}{$\begin{array}{c}\text { Overall } \\
(\mathrm{N}=23,645)\end{array}$} & \multicolumn{5}{|c|}{ Patients classified as_TRD $(\mathrm{N}=\mathbf{2 , 6 8 4})$} & \multicolumn{3}{|c|}{$\begin{array}{c}\text { Overall } \\
(\mathbf{N}=23,645)\end{array}$} & \multicolumn{4}{|c|}{$\begin{array}{l}\text { Patients classified as TRD } \\
(\mathrm{N}=\mathbf{2 , 6 4 8 )}\end{array}$} & \multirow[b]{2}{*}{ Sig } \\
\hline & $\mathbf{N}$ & $\%$ & $\mathbf{N}$ & Row\% & $\begin{array}{c}\text { Chi sq } \\
\text { statistic }\end{array}$ & $P$ value & Sig & $\begin{array}{l}\text { Characterist } \\
\text { ics }\end{array}$ & $\mathbf{N}$ & $\%$ & $\mathbf{N}$ & Row\% & $\begin{array}{l}\text { Chi sq } \\
\text { statistic }\end{array}$ & $P$ value & \\
\hline Index Year & & & & & 10.512 & 0.005 & *** & Obesity diagn & & & & & 1.479 & 0.224 & \\
\hline $2008-2010$ & 6257 & 26.5 & 747 & 11.9 & & & & Yes & 3842 & 16.2 & 458 & 11.9 & & & \\
\hline 2011-2013 & 7077 & 29.9 & 845 & 11.9 & & & & No & 19803 & 83.8 & 2226 & 11.2 & & & \\
\hline 2014-2016 & 10311 & 43.6 & 1092 & 10.6 & & & & Sleep disorde & & & & & 83.738 & $<0.001$ & $* * *$ \\
\hline Region & & & & & 6.289 & 0.098 & & Yes & 5445 & 23 & 806 & 14.8 & & & \\
\hline Northeast & 2170 & 9.2 & 278 & 12.8 & & & & No & 18200 & 77 & 1878 & 10.3 & & & \\
\hline Midwest & 5871 & 24.8 & 673 & 11.5 & & & & Tobacco use d & rder & & & & 25.03 & $<0.001$ & $* * *$ \\
\hline South & 9544 & 40.4 & 1076 & 11.3 & & & & Yes & 2399 & 10.1 & 346 & 14.4 & & & \\
\hline West & 6060 & 25.6 & 657 & 10.8 & & & & No & 21246 & 89.9 & 2338 & 11 & & & \\
\hline Sex & & & & & 8.181 & 0.004 & $* *$ & Alcohol use dí & der & & & & 11.563 & $<0.001$ & $* * *$ \\
\hline Female & 17340 & 73.3 & 2030 & 11.7 & & & & Yes & 606 & 2.6 & 95 & 15.7 & & & \\
\hline Male & 6305 & 26.7 & 654 & 10.4 & & & & No & 23039 & 97.4 & 2589 & 11.2 & & & \\
\hline Age group & & & & & 57.418 & $<0.001$ & $* * *$ & Drug use diso & & & & & 72.05 & $<0.001$ & $* * *$ \\
\hline $18-34$ years & 3127 & 13.2 & 351 & 11.2 & & & & Yes & 975 & 4.1 & 193 & 19.8 & & & \\
\hline $35-44$ years & 3616 & 15.3 & 470 & 13 & & & & No & 22670 & 95.9 & 2491 & 11 & & & \\
\hline $45-54$ years & 4706 & 19.9 & 596 & 12.7 & & & & Polypharmac & 5 medic & & & & 233.981 & $<0.001$ & $* * *$ \\
\hline $55-64$ years & 4468 & 18.9 & 554 & 12.4 & & & & Yes & 10529 & 44.5 & 1566 & 14.9 & & & \\
\hline$\geq 65$ years & 7728 & 32.7 & 713 & 9.2 & & & & No & 13116 & 55.5 & 1118 & 8.5 & & & \\
\hline Medicare Enrollm & ent & & & & 1.718 & 0.19 & & NSAID use & & & & & 10.783 & 0.001 & $* *$ \\
\hline Commercial & 14216 & 60.1 & 1645 & 11.6 & & & & Yes & 6036 & 25.5 & 755 & 12.5 & & & \\
\hline $\begin{array}{l}\text { Medicare } \\
\text { Advantage }\end{array}$ & 9429 & 39.9 & 1039 & 11 & & & & No & 17609 & 74.5 & 1929 & 11 & & & \\
\hline $\begin{array}{l}\text { Insurance plan } \\
\text { type }\end{array}$ & & & & & 2.064 & 0.356 & & Opioid use & & & & & 5.188 & 0.023 & * \\
\hline $\mathrm{HMO}$ & 7439 & 31.5 & 819 & 11 & & & & Yes & 6789 & 28.7 & 760 & 12.6 & & & \\
\hline PPO/POS & 11317 & 47.9 & 1319 & 11.7 & & & & No & 16856 & 71.3 & 1790 & 11.5 & & & \\
\hline
\end{tabular}




\begin{tabular}{|c|c|c|c|c|c|c|c|c|c|c|c|c|c|c|c|}
\hline Other & 4889 & 20.7 & 546 & 11.2 & & & & \multicolumn{5}{|c|}{ Beta blocker use } & \multirow[t]{2}{*}{15.698} & \multirow[t]{2}{*}{$<0.001$} & \multirow[t]{2}{*}{$* * *$} \\
\hline Back/neck pain & & & & & 30.475 & $<0.001$ & $* * *$ & Yes & 4795 & 20.3 & 622 & 13 & & & \\
\hline Yes & 16845 & 71.2 & 2034 & 12.1 & & & & No & 18850 & 79.7 & 2062 & 10.9 & & & \\
\hline No & 6800 & 28.8 & 650 & 9.6 & & & & Statin use & & & & & 0.006 & 0.938 & \\
\hline $\begin{array}{l}\text { Headache/ } \\
\text { migraine }\end{array}$ & & & & & 48.543 & $<0.001$ & $* * *$ & Yes & 6557 & 27.7 & 746 & 11.4 & & & \\
\hline Yes & 7849 & 33.2 & 1051 & 13.4 & & & & No & 17088 & 72.3 & 1938 & 11.3 & & & \\
\hline No & 15796 & 66.8 & 1633 & 10.3 & & & & \multicolumn{5}{|c|}{ Benzodiazepine use } & 144.594 & $<0.001$ & $* * *$ \\
\hline $\begin{array}{l}\text { Arthritis/Joint } \\
\text { Pain }\end{array}$ & & & & & 3.635 & 0.057 & & Yes & 6550 & 27.7 & 1006 & 15.4 & & & \\
\hline Yes & 21535 & 91.1 & 2471 & 11.5 & & & & No & 17095 & 72.3 & 1678 & 9.8 & & & \\
\hline No & 2110 & 8.9 & 213 & 10.1 & & & & \multicolumn{5}{|c|}{ Corticosteroid use } & 3.463 & 0.063 & \\
\hline \multicolumn{5}{|c|}{ Neuropathic pain/other chronic pain } & 48.733 & $<0.001$ & $* * *$ & Yes & 2697 & 11.4 & 335 & 12.4 & & & \\
\hline Yes & 5417 & 22.9 & 758 & 14 & & & & No & 20948 & 88.6 & 2349 & 11.2 & & & \\
\hline No & 18228 & 77.1 & 1926 & 10.6 & & & & \multicolumn{5}{|c|}{ Psychotherapy before MDD } & 36.021 & $<0.001$ & $* * *$ \\
\hline No. of CNPC & & & & & 95.738 & $<0.001$ & $* * *$ & Yes & 1132 & 4.8 & 191 & 16.9 & & & \\
\hline 1 & 5217 & 22.1 & 469 & 9 & & & & No & 22513 & 95.2 & 2493 & 11.1 & & & \\
\hline 2 & 10469 & 44.3 & 1095 & 10.5 & & & & \multicolumn{5}{|c|}{ Psychotherapy after MDD $\dagger$} & 182.284 & $<0.001$ & $* * *$ \\
\hline$\geq 3$ & 7959 & 33.7 & 1120 & 14.1 & & & & Yes & 4653 & 19.7 & 790 & 17 & & & \\
\hline Anxiety & & & & & 137.811 & $<0.001$ & $* * *$ & No & 18992 & 80.3 & 1894 & 10 & & & \\
\hline Yes & 6520 & 27.6 & 996 & 15.3 & & & & \multicolumn{5}{|c|}{ Mental health specialist visit $\dagger$} & 437.948 & $<0.001$ & $* * *$ \\
\hline No & 17125 & 72.4 & 1688 & 9.9 & & & & Yes & 5536 & 23.4 & 882 & 20.5 & & & \\
\hline Dementia & & & & & 23.516 & $<0.001$ & $* * *$ & No & 18109 & 76.6 & 1802 & 9.3 & & & \\
\hline Yes & 1058 & 4.5 & 169 & 16 & & & & \multicolumn{5}{|c|}{ Primary care provider visit $\dagger$} & 12.887 & $<0.001$ & $* * *$ \\
\hline No & 22587 & 95.5 & 2515 & 11.1 & & & & Yes & 19892 & 84.1 & 2194 & 11 & & & \\
\hline Other mental ill & & & & & 78.067 & $<0.001$ & $* * *$ & No & 3753 & 15.9 & 490 & 13.1 & & & \\
\hline Yes & 703 & 3 & 153 & 21.8 & & & & \multicolumn{5}{|c|}{ Any Inpatient hospitalizations $\dagger$} & 108.362 & $<0.001$ & $* * *$ \\
\hline No & 22942 & 97 & 2531 & 11 & & & & Yes & 2593 & 11 & 453 & 17.5 & & & \\
\hline Respiratory con & tions & & & & 33.582 & $<0.001$ & $* * *$ & No & 21052 & 89 & 2231 & 10.6 & & & \\
\hline Yes & 3003 & 12.7 & 435 & 14.5 & & & & \multicolumn{5}{|c|}{ MDD related Inpatient hospitalizations $\dagger$} & 94.058 & $<0.001$ & *** \\
\hline No & 20642 & 87.3 & 2249 & 10.9 & & & & Yes & 1116 & 4.7 & 227 & 20.3 & & & \\
\hline Cardiovascular & nditions & & & & 1.018 & 0.313 & & No & 22529 & 95.3 & 2457 & 10.9 & & & \\
\hline
\end{tabular}




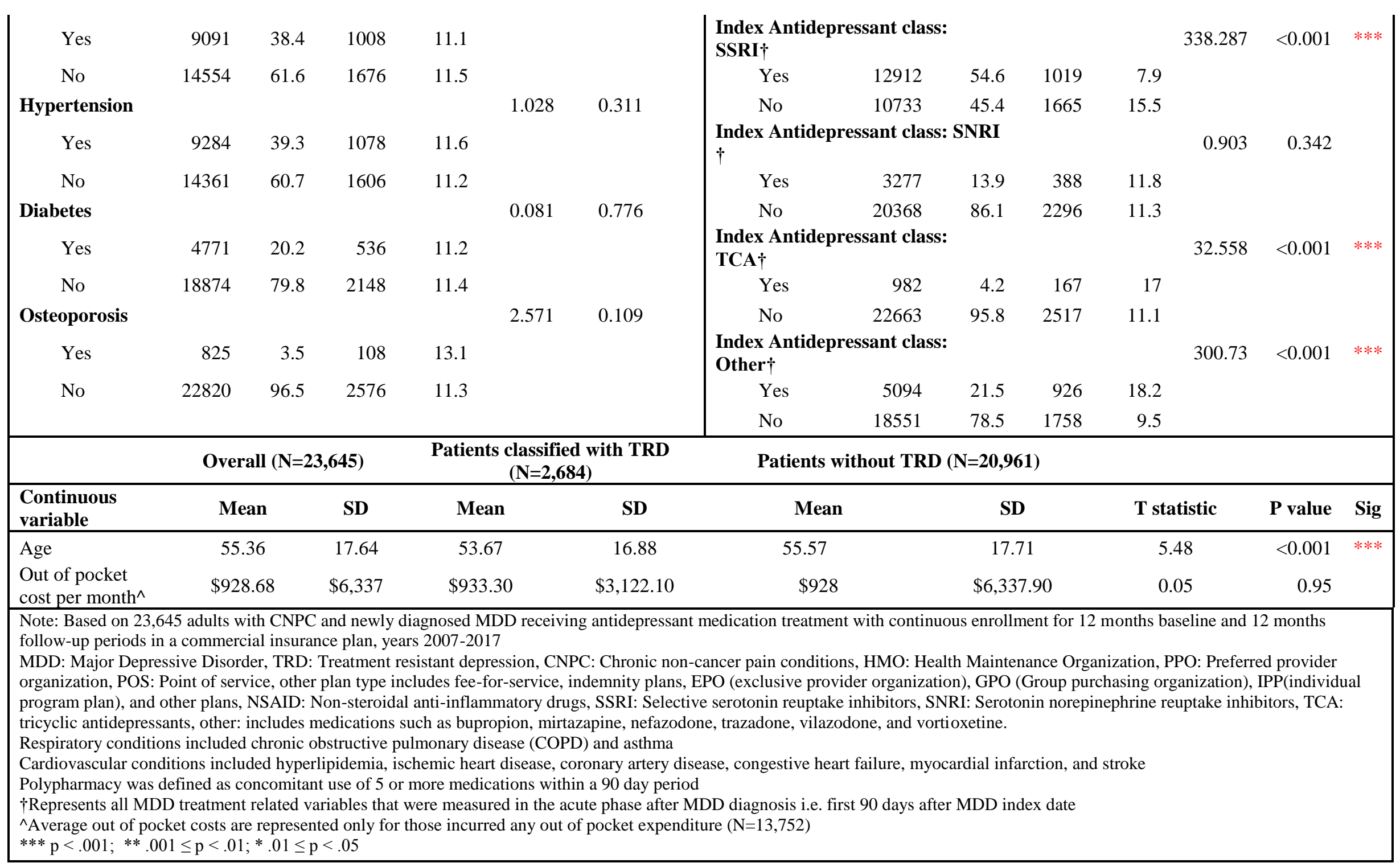


Figure 2.2 Variable importance values for top 10 variables of TRD among patients with CNPC and newly diagnosed MDD obtained from Machine Learning Approaches: A) Logistic regression B) Random forest models

Figure 2.2.A

\# CNPC conditions $(\geq 3)$

Index Antidepressant class:other

Any IP hospitalization

Sex (female)

Index Antidepressant class:SSRI

Age (35-44 years)

Anxiety

Psychotherapy after MDD

Polypharmacy

Mentalhealth specialist visit
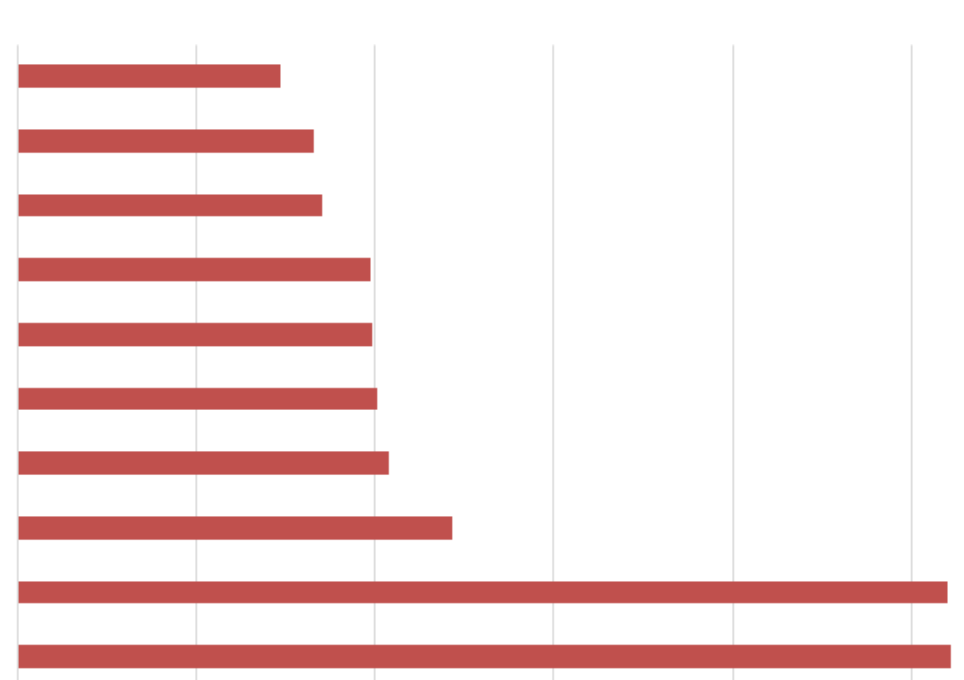

0

4

6

8

10

Variable Importance from Logistic regression

Figure 2.2.B

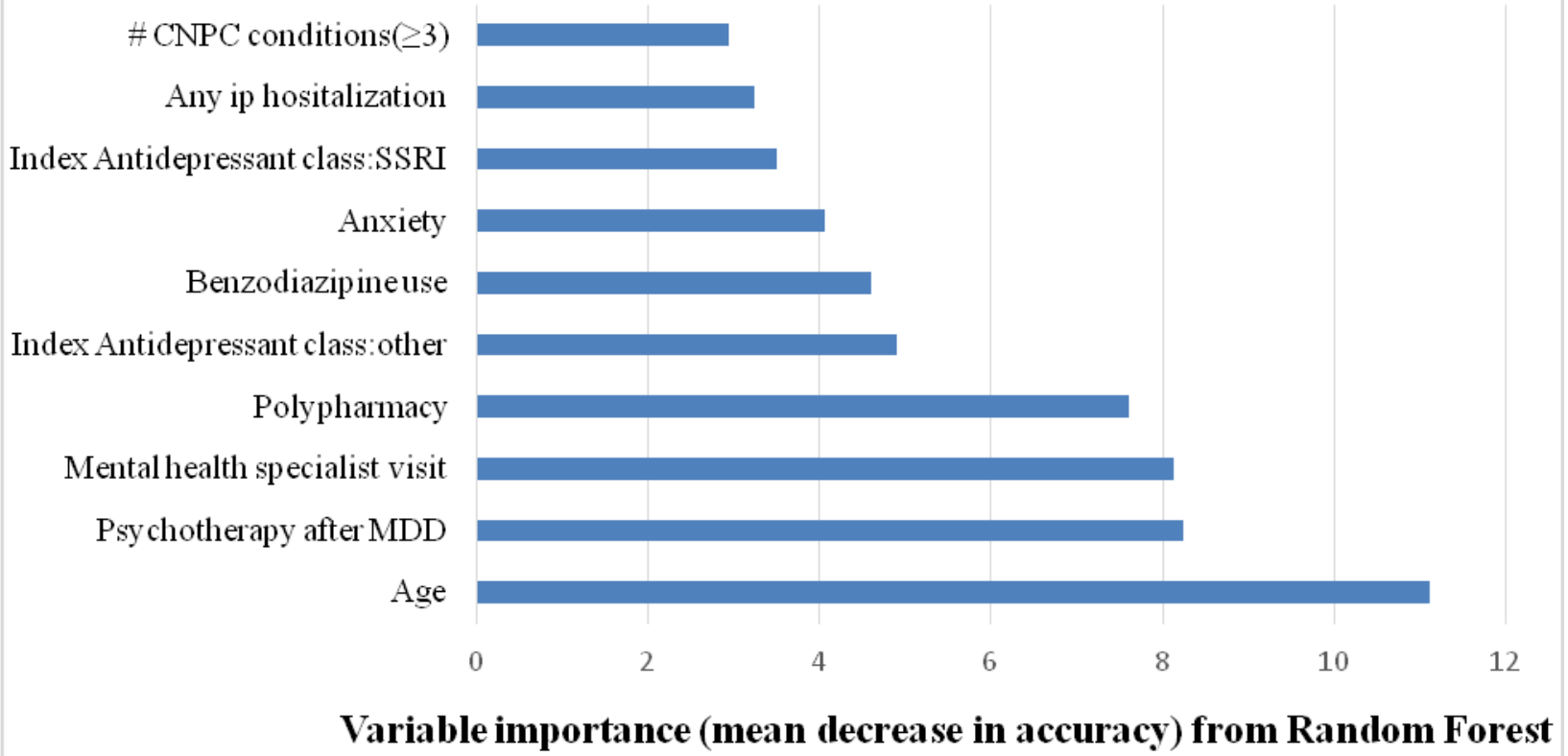

Note: Predictors are listed on the $\mathrm{Y}$ axis by increasing impact of importance from the top to bottom.

CNPC: Chronic non-cancer pain conditions, IP: Inpatient, SSRI: Selective serotonin reuptake inhibitors 


\begin{tabular}{|lrrr|}
\hline \multicolumn{4}{|c|}{$\begin{array}{c}\text { Table 2.3. Adjusted Odds Ratio and 95\% Confidence Intervals for Important Predictors of } \\
\text { TRD from Logistic Regression Model for Adults with CNPC and Newly Diagnosed MDD } \\
\text { Optum Clinformatics Real-World De-identified Data, 2007-2017 }\end{array}$} \\
\hline Predictor & AOR & $\mathbf{9 5 \%}$ CI & P value \\
\hline Mental health provider visit (yes vs. no) & 1.93 & $1.70-2.18$ & $<0.001$ \\
Polypharmacy (yes vs. no) & 1.81 & $1.62-2.03$ & $<0.001$ \\
Use of psychotherapy after MDD diagnosis (yes vs. & 1.36 & $1.20-1.55$ & $<0.001$ \\
no) & 1.27 & $1.13-1.41$ & $<0.001$ \\
Anxiety (yes vs. no) & 1.41 & $1.19-1.66$ & 0.002 \\
Age (35-44 years vs. $\geq$ 65 years) $\dagger$ & 1.32 & $1.1-1.58$ & $<0.001$ \\
Age (18-34 years vs. $\geq$ 65 years) $\dagger$ & 0.69 & $0.60-0.79$ & $<0.001$ \\
Index Antidepressant class: SSRI (yes vs. no) & 1.27 & $1.13-1.42$ & $<0.001$ \\
Sex (female vs. male) & 1.26 & $1.11-1.48$ & $<0.001$ \\
Any IP hospitalization (yes vs. no) & 1.38 & $1.12-1.7$ & $<0.001$ \\
Index Antidepressant class: other (yes vs. no) & 1.23 & $1.09-1.39$ & $<0.001$ \\
No. of CNPC conditions ( $\geq 3$ vs. 1) & 1.18 & $1.05-1.32$ & $<0.001$ \\
Benzodiazepine use (yes vs. no) & &
\end{tabular}

Note: Based on 23,645 adults with CNPC and newly diagnosed MDD receiving antidepressant medication treatment with continuous enrollment for 12 months baseline and 12 months follow-up periods in a commercial insurance plan, years 2007-2017

MDD: Major Depressive Disorder, TRD: Treatment resistant depression, CNPC: Chronic non-cancer pain conditions, SSRI: Selective serotonin reuptake inhibitors, other antidepressants: includes medications such as bupropion, mirtazapine, nefazodone, trazadone, vilazodone, and vortioxetine, IP: Inpatient, AOR:

Adjusted odds ratio, CI: Confidence Interval

$\dagger$ Only age groups with $p<0.01$ is presented 


\section{CHAPTER 3}

\section{Economic Burden of Treatment-Resistant Depression among Adults with Chronic Non- Cancer Pain Conditions and Major Depressive Disorder}

\subsection{Abstract}

Objective: Treatment-resistant depression (TRD) in adults with chronic non-cancer pain conditions (CNPC) can amplify the economic burden. This study examined the impact of TRD on direct total and MDD-related healthcare resource utilization (HRU) and costs among patients with CNPC and MDD.

Methods: The study used a retrospective longitudinal cohort design and employed a claimsbased algorithm to identify adults with TRD from a US claims database (January 2007- June 2017). HRU and costs were measured over a 12-month period after TRD/non-TRD index date. Counterfactual recycled predictions from multivariable generalized linear models were used to examine associations between TRD and annual HRU and costs. Post-regression linear decomposition identified differences in patient-level factors between TRD and no TRD group that contributed to the excess economic burden of TRD.

Results: Of the 21,180 adults with CNPC and MDD, 10.1\% were identified as having TRD. TRD patients had significantly higher HRU, translating into higher average total costs ( $\left.\$ 21,015_{\text {TRD Vs }} \$ 14,712_{\text {No TRD }}\right)$ and MDD-related costs $\left(\$ 1,201_{\text {TRD }}\right.$ vs $\$ 471_{\text {No TRD }}$ ) compared to non-TRD counterparts (all $\mathrm{P}<0.001$ ). TRD patients had significantly higher number of inpatient visits $(\mathrm{IRR}=1.30 .95 \% \mathrm{CI}=1.14-1.47 ; \mathrm{P}<0.001)$ than non-TRD patients. Overall, $46 \%$ of the excess total costs were explained by differences in patient level characteristics such as polypharmacy, number of CNPC, anxiety, sleep, and substance use disorders between the TRD and no TRD groups. 
Conclusion: TRD poses a significant direct economic burden among adults with CNPC and MDD. Excess healthcare costs may potentially be reduced by providing timely interventions for several modifiable risk factors.

\subsection{Introduction}

Major Depressive Disorder (MDD) is a highly prevalent mental disorder and presents a substantial economic burden. ${ }^{8,69}$ In 2015, the incremental economic burden of MDD in the United States (US) was estimated to be as high as $\$ 210.5$ billion, with $\$ 98.9$ billion spent on direct healthcare costs. ${ }^{8}$ Co-existence of MDD and chronic conditions has been reported to be the main driver of this high economic burden. ${ }^{8}$ For example, MDD is associated with $50 \%$ to $100 \%$ higher medical costs among patients with co-existing chronic conditions even after risk adjustments..$^{10}$ Among commonly co-occurring chronic conditions with MDD, chronic noncancer pain conditions (CNPC) is of particular interest because of the high prevalence and well documented bi-directional relationship between MDD and pain., ${ }^{73,14}$ The prevalence of MDD in patients with CNPC can be as high as $85 \% .{ }^{7} \mathrm{CNPC}$ and MDD are inextricably linked in terms of pain, biological pathways, and pathophysiology ${ }^{14,15}$ which may contribute to the excess economic burden of MDD. A study documenting the excess costs of MDD across 11 chronic conditions including chronic non-cancer pain conditions (CNPC) such as osteoarthritis, joint pain, back pain, and headache highlighted a substantial economic burden of MDD. ${ }^{102} \mathrm{~A}$ handful of studies have also documented the excess economic burden of MDD among adults with CNPC, ${ }^{47,103}$ which may be in part explained by the additive adverse effects of MDD and CNPC on clinical outcomes. ${ }^{103,104}$

Additionally, the economic burden of MDD and CNPC may be amplified for patients whose MDD is resistant to depression treatment. ${ }^{11}$ It has been documented that patients with 
CNPC may not respond adequately to antidepressants, a major modality of depression treatment, which can subsequently increase the risk of treatment-resistant depression (TRD). ${ }^{11}$ TRD is primarily MDD in which patients do not achieve remission even after undergoing multiple repeated and vigorous antidepressant treatment trials. ${ }^{21,105}$ Studies have highlighted a high prevalence of TRD among adults with CNPC. ${ }^{20,22,24,29}$ As adults with TRD tend to have much longer episodes of depression, higher symptom severity, and higher healthcare resource utilization (HRU), the association of TRD with high economic burden has been documented in studies that are not specific to CNPC. ${ }^{20,27,106}$ For example, adults with TRD had two times higher direct healthcare costs and higher HRU as compared to adults without TRD. ${ }^{25,41,42,44,46}$ However, to-date, no study has examined the economic burden of TRD among adults with CNPC and MDD.

Estimating the economic burden of TRD in CNPC patients is important for several reasons: 1) Many policy initiatives have shifted focus to the pursuit of triple aim of improving the health of patients, quality of care and reducing healthcare costs, ${ }^{48}$ which requires identification of high-cost, high-need patients, ${ }^{48}$ 2) Payment reforms have focused on reimbursement methods such as bundled payments for episodes of care which require risk adjustment to account for differences in beneficiary-case mix. An up to date risk-adjusted estimate of economic burden for those with MDD should also include excess costs due to TRD; 3) Helps in understanding their unmet needs, demonstrate the value for the need of targeted interventions and increased investments in mental health resources in this population; and 4) quantification of incremental burden of illness of TRD helps in translating the burden of illness into dollar terms, the universal language for decision making. ${ }^{49}$ 
Furthermore, TRD can be influenced by many factors..$^{20,27,29,50}$ Therefore, it is important to estimate how much of the excess economic burden can be explained by differences in patientlevel characteristics between those with TRD and non-TRD patients. Understanding factors that can contribute the excess economic burden of TRD can aid payers in identifying major cost drivers. Therefore, the main objectives of this study were: to estimate the excess economic burden (direct healthcare utilization and costs) associated with TRD and to examine the extent to which differences in patient factors between TRD and no TRD groups contribute to the excess cost burden of TRD by using a post-regression linear decomposition approach among commercially insured adults with CNPC and newly diagnosed MDD.

\subsection{Methods}

\section{Data Source}

This study was conducted using health insurance claims data (January 2007 through June 2017) from the de-identified Optum ${ }^{\circledR}$ Clinformatics ${ }^{\circledR}$ Data Mart. ${ }^{67}$ We used the $10 \%$ random sample of Optum data, which is a geographically diverse database, spanning across all 50 states. It contains information on diagnoses, procedures, treatment, medication use, and costs derived from inpatient, outpatient and pharmacy claims. Demographic characteristics (age, sex, state of residence), and health plan information are also routinely collected during health insurance enrollment. ${ }^{67}$

\section{Study Design}

This study utilized a retrospective longitudinal cohort design. The study period consisted of cohort identification, baseline, and follow-up periods. The cohort identification period was used to identify adults with CNPC and newly diagnosed MDD. The baseline period, defined as 12 months before TRD index date (randomly imputed pseudo-index date for non-TRD) was used to identify independent variables, including baseline HRU and costs. The follow-up period (12 
months after TRD index/pseudo-index date) was used to compute the economic outcomes (HRU and direct costs).

\section{Cohort Selection}

The study cohort comprised adults with CNPC and newly diagnosed MDD (identified using ICD9/10 codes) between January 2008 and June 2016 (Appendix 7.1). Adults with CNPC were required to have at least two or more healthcare encounters during the cohort identification period (i.e 12 months before the first observed MDD diagnosis). CNPC was identified using an extensive list of conditions for which pain was severe enough to classify them as chronic pain condition. ${ }^{80-82}$ To ensure that the episode of MDD was newly diagnosed, we used a validated algorithm of including only those who did not have any MDD diagnosis or antidepressant prescription in 180 days prior to the MDD diagnosis date. ${ }^{78,79}$

We used the following additional inclusion-exclusion eligibility criteria to identify the study cohort:1) adult patients (age $\geq 18$ years) with at least one antidepressant prescription following MDD diagnosis, 2) no diagnosis for cancer throughout the study period, 3) consistent with prior studies, those with a diagnosis of schizophrenia, schizoaffective disorder, other psychosis, manic depression or bipolar disorders were excluded, ${ }^{22,23} 4$ ) continuous enrollment during the 12 months before MDD identification and 24 months after MDD identification. (See Figure 3.1 for number of individuals eliminated at each step of application of inclusion-exclusion criteria). The final sample consisted of 21,180 adults with CNPC and newly diagnosed MDD.

\section{Measures}

\section{Key Independent Variable: Treatment-Resistant Depression}

We used a claims-based algorithm that was developed using the Massachusetts General Hospital (MGH) clinical staging method. ${ }^{23,61}$ This claims-based algorithm has been previously 
used to estimate the cost burden associated with TRD. ${ }^{23}$ Although previous studies using administrative claims data to study TRD has defined TRD using different definitions, ${ }^{22,23,41,42}$ we chose the MGH-based algorithm because it can be readily translated to prescription claims information. Furthermore, it encompasses the four main strategies to overcome lack of antidepressant treatment response, i.e. optimization, switching, combination, or augmentation. ${ }^{62}$ We defined TRD using a point-based algorithm; one point was assigned for each adequate antidepressant trial (i.e., 2 or more fills of the same antidepressant), and half a point for each optimization strategy. The optimization strategies included extended duration (i.e. at least 3 fills of the same antidepressant), an upward titration in dose, and augmentation with an atypical antipsychotic, mood stabilizer, or thyroid hormone. Individuals exceeding three points were considered to have TRD. ${ }^{23}$ Those using electroconvulsive therapy (ECT) were considered to have TRD as ECT is often used as a late-stage treatment for TRD. ${ }^{7}$

\section{Study Outcomes}

\section{HRU and Direct Healthcare Costs}

Direct costs and HRU (here-after referred as costs and HRU) included both all-cause and MDD-related utilization and costs. For HRU, dependent variables were counts of total and MDD-related inpatient admissions, outpatient visits, and emergency department (ED) visit. Claims data from Optum are verified and adjusted with a standard pricing methodology to account for variations in allowed payments across health plans and provider contracts prior to inclusion in the Data Mart dataset. Total costs included allowed payments for inpatient stays, facility outpatient visits, professional services, and prescription drugs from the insurer. Allowed payments from the insurer were estimated and normalized to 2018 US dollars using the cost factors and standardized prices provided by Optum Clinformatics ${ }^{\circledR}$ DataMart. MDD-related 
costs and use were identified using the primary or secondary ICD-9/10 CM diagnosis codes indicating MDD (see Appendix 7.1 for code definitions). Utilization and costs were summed over a period of 12 months following TRD index/pseudo-index date

\section{Other independent variables (measured at baseline)}

The selection of other independent variables that could impact economic outcomes was guided by the Modified Andersen's Behavioral Framework of Health Services Use. ${ }^{64}$ The model posits that the health services costs and utilization of an individual is primarily a function of predisposing factors (e.g., age, sex), enabling factors (e.g. type of insurance plan, Medicare advantage enrollment), need factors (e.g. chronic conditions, obesity, sleep disorders, tobacco use disorder, and drug/alcohol use disorder, polypharmacy, benzodiazepine use, and use of pain medication such as opioids and NSAIDs), and external environment (e.g. geographical region). Chronic conditions included comorbid anxiety disorders, sum of CNPC conditions, and number of chronic physical conditions other than CNPC. Baseline cost and resource use was also measured.

\section{Statistical Analyses}

Baseline patient characteristics were compared between patients with and without TRD using chi-square test for categorical variables and t-test for continuous variables. Adjusted associations between TRD and HRU in the follow-up period was assessed using multivariable negative binomial regressions and the results are reported using incidence rate ratios (IRR) and 95\% confidence intervals (CI). The adjusted associations between TRD and healthcare costs were evaluated using two-part models (TPM). Because healthcare and MDD-related costs had zero mass (> 5\%), we used two part models for all cost analyses ${ }^{107}$ The first part of the model used a logistic regression to assess the association between TRD and any positive cost and the 
second part of the model used generalized linear models (GLM) with a gamma distribution and log-link function to handle positively skewed cost distribution. Rather than simply comparing the expenditures between the groups for a reference case (i.e. marginal effects) robust estimates for incremental costs between TRD and non-TRD groups were obtained using the counterfactual recycled prediction technique from parameter estimates of GLM. ${ }^{108}$ Cost differences, p-values, and their adjusted $95 \%$ confidence intervals were obtained from nonparametric bootstrap procedures (500 replications). We present estimates from two models, one with risk-adjustment for the predisposing, need, and external environmental factors described above and other with additional risk adjustment for healthcare costs.

To estimate the extent to which differences in patient-level characteristics between TRD and no- TRD groups contribute to the excess total costs associated with TRD, we use the Blinder-Oaxaca linear decomposition approach. ${ }^{109,110,111}$ This technique assesses how much of the difference in expenditures between the two groups may be due to observable or explained factors (e.g. the predisposing, enabling, need, and external environmental factors described above) versus unobservable or unexplained factors (e.g. lifestyle difference, social support, education, income).The explained portion can be interpreted as counter-factual scenario (i.e. estimated differences in cost burden if adults with TRD had the same characteristics as adults without TRD). All data management and analyses were performed with SAS 9.4 (SAS Institute Inc., Cary, NC) and Stata 14 (StataCorp LLC, College Station, TX).

\subsection{Results}

\section{Sample Description}

Of a total of 21,180 adults with CNPC and newly diagnosed MDD, 10.1\% (N=2,147) adults transitioned from MDD to TRD within one year of MDD diagnosis. On average patients 
identified as TRD were 53 years old and were predominately females (77\%). The description of study sample characteristics and subgroup differences in characteristics of adults with and without TRD are presented in Table 3.1. Significant differences were observed in predisposing and need factors between TRD and non-TRD groups. For example, a significantly higher proportion of TRD patients had higher rates of polypharmacy $(69.4 \%$ vs. $49.6 \%)$, had $\geq 3 \mathrm{CNPC}$ (40.6\% vs. $32.1 \%)$, had comorbid anxiety disorders (55.7\% vs. $15.3 \%)$, had sleep disorders (33.6\% vs. $23.7 \%)$, and drug/alcohol use disorders (15.9\% vs. $8.1 \%)$ as compared to non-TRD patients (all $\mathrm{P}<0.001$ ). The rates of baseline HRU were significantly higher among adults with TRD as compared to those without TRD (all $\mathrm{P}<0.001$ ) (Table 3.1).

\section{HRU}

Unadjusted (Appendix 7.8) and adjusted rates (Figure 3.2) of HRU during the follow-up period were higher for those with TRD as compared to those without TRD. Adults with TRD had significantly higher number of inpatient $(\mathrm{IRR}=1.30,95 \% \mathrm{CI}=1.14-1.47)$ and $\mathrm{ED}$ visits $(\mathrm{IRR}=1.21,95 \% \mathrm{CI}=1.10-1.34)$ and higher MDD-related healthcare utilization (e.g. 3.0 and 2.23 times the inpatient and ED visit rate) as compared to adults without TRD.

\section{Healthcare Costs}

We observed that the mean unadjusted total costs during the 12-month follow-up period (Table 3.2) were higher for adults with TRD as compared to those without TRD $\left(\$ 21,015_{\mathrm{TRD}}\right.$ and $\$ 14,712_{\text {No TRD }}$, with an excess per patient cost of $\$ 6,303(\mathrm{P}<0.001)$. Prescription drug costs accounted for $37.6 \%$, inpatient services for $30.7 \%$, outpatient services for $21.3 \%$ and ED use for the remaining $10.6 \%$ of the excess TRD healthcare costs (can be calculated from Table 3.2). Similarly, adults with TRD had significantly higher unadjusted MDD-related costs as compared to adults without TRD ( $\$ 1,201$ vs. $\$ 471, \mathrm{P}<0.001)$. Adults with TRD had significantly higher 
costs for each component of all-cause and MDD-related costs as compared to adults without TRD (Table 3.2).

Adjusted all-cause and MDD-related healthcare costs remained significantly higher (Model 1) for those with TRD as compared to adults without TRD, yielding an excess cost estimate of $\$ 3,388$ for all-cause cost and $\$ 394$ for MDD-related total costs. After additionally adjusting for baseline healthcare costs (Model 2), the excess TRD costs reduced to $\$ 2,025$ (allcause) and \$296 (MDD-related). For all components of all-cause and MDD-related costs, those with TRD had higher costs compared to those without TRD $(\mathrm{P}<0.001)$ (Table 3.2).

Table 3.3 summarizes the results from the post-regression linear decomposition. Nearly half of the difference $(46 \%)$ in total all-cause costs was explained by differences in the baseline characteristics between the TRD and no TRD groups. This means that if the TRD group were to have the same distribution of characteristics as the no-TRD group, $46 \%$ of the excess cost burden can be reduced. Among all patient level factors, need factors, specifically differences in polypharmacy rates contributed to over half (55.7\%) of the explained portion of the total costs. Other contributors to the excess cost burden were: number of CNPC (11.24\%), tobacco and drug use disorders (10.99\%), and presence of sleep and anxiety disorders $(8.17 \%)$. When baseline costs were added to the model, it explained $65 \%$ of the difference in total healthcare costs between the two groups (data not provided in tabular form).

\section{Secondary Analysis}

We also conducted a series of secondary analysis to ensure robustness of the association between TRD and economic outcomes. First, we used inverse probability of treatment weighting (IPTW) to account for observable selection bias between TRD and no TRD groups. ${ }^{112}$ IPTWs were derived from a multivariable logistic regression, in which presence or absence of TRD was 
the dependent variable. Second, we assessed the association between TRD and total all-cause and MDD related healthcare costs over time. We captured costs during the observation period in 120-day periods over six intervals. In these models, polypharmacy ( $>5$ concomitant use of medications in a period of 90 days), use of benzodiazepines and use of pain -related medications such as opioids and NSAIDs were measured in each of the 120-day periods. To account for correlated errors terms due to repeated measures, we used generalized linear mixed model (GLMM) with gamma distribution and log link.

IPTW adjustments (Appendix 7.7) and GLMM yielded results similar to the primary analyses (Appendix 7.9). For example, GLMM analysis indicated that on average, patients who transitioned to TRD had significantly higher all-cause total costs $(\$ 1746.5 ; \beta=0.31$; $\mathrm{P}$ value $<0.001)$ and MDD-related costs $(\beta=1.38 ; \mathrm{P}$ value $<0.001)$ at every time point in the baseline and follow-up periods even after adjusting for patient characteristics (Appendix 7.9).

\subsection{Discussion}

Approximately one in 10 adults with CNPC and newly diagnosed MDD were identified as having TRD, a finding consistent with some previously reported estimates of TRD from other claims-based studies (6\% to $15 \%) .{ }^{22,29,44,46}$ We observed that patients with TRD had approximately 1.4 times higher total all-cause healthcare costs than adults without TRD. Although, to date, no study has estimated excess economic burden of TRD among adults with CNPC, we provide some comparisons based on studies that used similar algorithms to identify TRD among in adults with MDD. Our adjusted total cost was similar to the estimates provided by Gibson et al., who reported that TRD was associated with $40 \%$ higher total healthcare costs among adults with MDD. ${ }^{23}$ The findings from the current study were robust to the different specifications of the models used in primary and sensitivity analysis. All models indicated 
significantly higher costs among adults with TRD compared to no TRD group. We observed a higher economic burden among TRD patients at every 120-day period over two years. A plausible reason may be the unremitting depressive state experienced by the TRD patients. Prior research suggests that TRD patients had persistent depressive symptoms for nearly $61 \%$ of the time over a three-year period. ${ }^{113}$ Therefore, our findings suggest that efforts in preventing transition from MDD to TRD by screening patients for risk factors of TRD such as presence of comorbid anxiety disorders, suicidal tendencies, hospitalization status, and high depression symptom severity $y^{26,27,29}$ can potentially reduce costs of MDD among adults with CNPC and newly diagnosed MDD.

In our study sample of adults with CNPC and newly diagnosed MDD, a significant portion of the total all-cause costs was accounted by prescription drug costs, a finding consistent with prior studies of TRD in commercially insured populations. ${ }^{22,42} \mathrm{We}$ also observed that polypharmacy rates from our study were significantly higher in the TRD group as compared to no TRD group (69.4\% vs 49.6\%). Furthermore, results from the linear decomposition method indicated that polypharmacy was a major driver of the excess total costs associated with TRD. Our findings suggest that if adults with TRD were to have the same polypharmacy rates as adults without TRD, the excess total cost burden associated with TRD as explained by all the measurable baseline factors can be reduced by more than half (55.7\%). Interventions that have shown to reduce the risk of polypharmacy such as systematic review of high risk medications (termed as "deprescribing"), medication therapy management, medication reconciliation, and using a multidisciplinary care approach ${ }^{114}$ have the potential to off-set the excess costs due to polypharmacy. Future studies on the cost of illness of TRD should use polypharmacy as a 
measure for risk-adjustment while presenting adjusted incremental cost estimates associated with TRD.

Inpatient costs also accounted for a substantial share of all-cause as well as MDD-related total healthcare costs. This is consistent with reports obtained from previous studies conducted among adults with MDD. ${ }^{22,25,42}$ The finding is also reflected by the significantly higher number of all-cause (IRR=1.30) as well as MDD-related inpatient visits (IRR=3.00) among adults with TRD as compared to those without TRD. A study by Crown et al. also reported that as compared to the TRD patients treated in outpatient care, hospitalized TRD patients had significantly higher MDD-related hospitalization as well as general medical costs. ${ }^{25}$ Although, we did not explore the reasons for all-cause or MDD-related hospitalizations, adults with TRD may have higher risk of hospitalization and inpatient costs due to higher polypharmacy rates and greater occurrence of chronic conditions such as number of CNPC, anxiety and substance use disorders as observed in our study. It has been documented that polypharmacy can elevate risks of adverse drug-drug interactions, which can lead to increased rates of hospitalizations and inpatient costs. ${ }^{115} \mathrm{TRD}$ patients may also engage in high-risk behavior such as substance use and suicidal attempts, at a higher rate than no TRD patients, ${ }^{116,117}$ which are all factors that increases the risk of hospitalization and re-admissions. ${ }^{118,119}$

We also found that multimorbidity (example: number of CNPC, presence of anxiety, sleep and substance use disorders) to be one of the major drivers of the excess total costs associated with TRD. Research has shown that multimorbidity has a profound impact on healthcare costs due to several reasons such as increased use of emergency department visits, adverse drug events due to polypharmacy, and complex disease trajectories due to interactions among chronic conditions. ${ }^{120}$ For example, adults with three or more CNPC may experience 
severe pain that may worsen clinical outcomes among adults with TRD. ${ }^{7}$ Co-management of these commonly co-occurring conditions is often difficult, and results in higher healthcare costs. ${ }^{121}$ Therefore, our findings suggests that the complex needs of patients with multimorbidity can be better managed through healthcare delivery models that use collaborative and multidisciplinary approaches. Such models have been shown to improve clinical outcomes, and reduce costs as compared to the usual source of care. ${ }^{122,123} 124$

\section{Strengths and Limitations}

To date, this is the first real-world study to quantify the direct economic burden of TRD among adults with CNPC and MDD; two very commonly co-occurring chronic conditions. The longitudinal study design with repeated measures allowed for an assessment of baseline as well as follow-up profiles in terms of utilization, expenditures as well as baseline patient characteristics. Further, MGH scoring algorithm to identify TRD allows for a nuanced and comprehensive assessment of TRD by accounting for number of failed adequate trials, treatment dose, titration, duration and augmentation. Additionally, the current study ensured robustness of study findings by adjusting for observed selection bias through IPTWs and by employing the use of counterfactual-recycled predictions in addition to the use of a two-part GLM model. This allowed us to obtain estimates for an average patient as compared to estimates obtained from a reference-case scenario using marginal effects derived from the two-part GLM regression models.

Although the insights from the current study are important, the limitations of the current study need to be considered while interpreting the study findings. TRD was defined using pharmacy claims and ECT use and excludes other clinical considerations such as type of symptoms, responsiveness to medical treatments, and tolerance to antidepressants. Further, we 
may not have truly captured the actual use of medication as we could observe only presence of prescription claims. It is also plausible that by limiting patients to only MDD diagnosis, we may have underestimated the prevalence of MDD because MDD may at times could be recorded with general depression code. As shown from the results of the decomposition analysis, absence of information on factors such as severity of MDD and pain, socioeconomic status, race/ethnicity, physical activity, and social support could contribute to the unexplained portion of the total costs differences between adults with and without TRD, which could impact the study findings. Our study estimated costs from a third-party payer perspective, therefore; indirect costs such as costs related to traveling, caregiving and loss of productivity have not been considered. In estimating costs associated with MDD, costs of prescription medications have not been considered as medications such as antidepressants and atypical antipsychotics were used in the algorithm to identify patients with TRD and these medications could also be used for other physical conditions such as pain, and insomnia. ${ }^{125}$ Finally, although our study was not limited to including only working-aged adults, our results may not be generalizable to other privately and publicly insured adults. Additionally, no direct inference should be made about the prevalence of TRD based on the size of the analytical sample which was selected based on several inclusion/exclusion criteria.

\subsection{Conclusion}

This is the first study to document the excess direct economic burden of TRD among adults with CNPC and newly diagnosed MDD. TRD in adults with CNPC and newly diagnosed MDD was associated with substantial all-cause and MDD-related costs and HRU. Estimates from our study can provide a benchmark of TRD costs for future cost-effectiveness studies and in estimating risk-adjusted cost for value-based reimbursement initiatives. Potential cost savings 
associated with managing TRD may stem from reducing the risk of polypharmacy, better comanagement of chronic pain and co-occurring substance use, anxiety, and sleep disorders. 
Figure 3.1. Schematic presentation of study sample selection

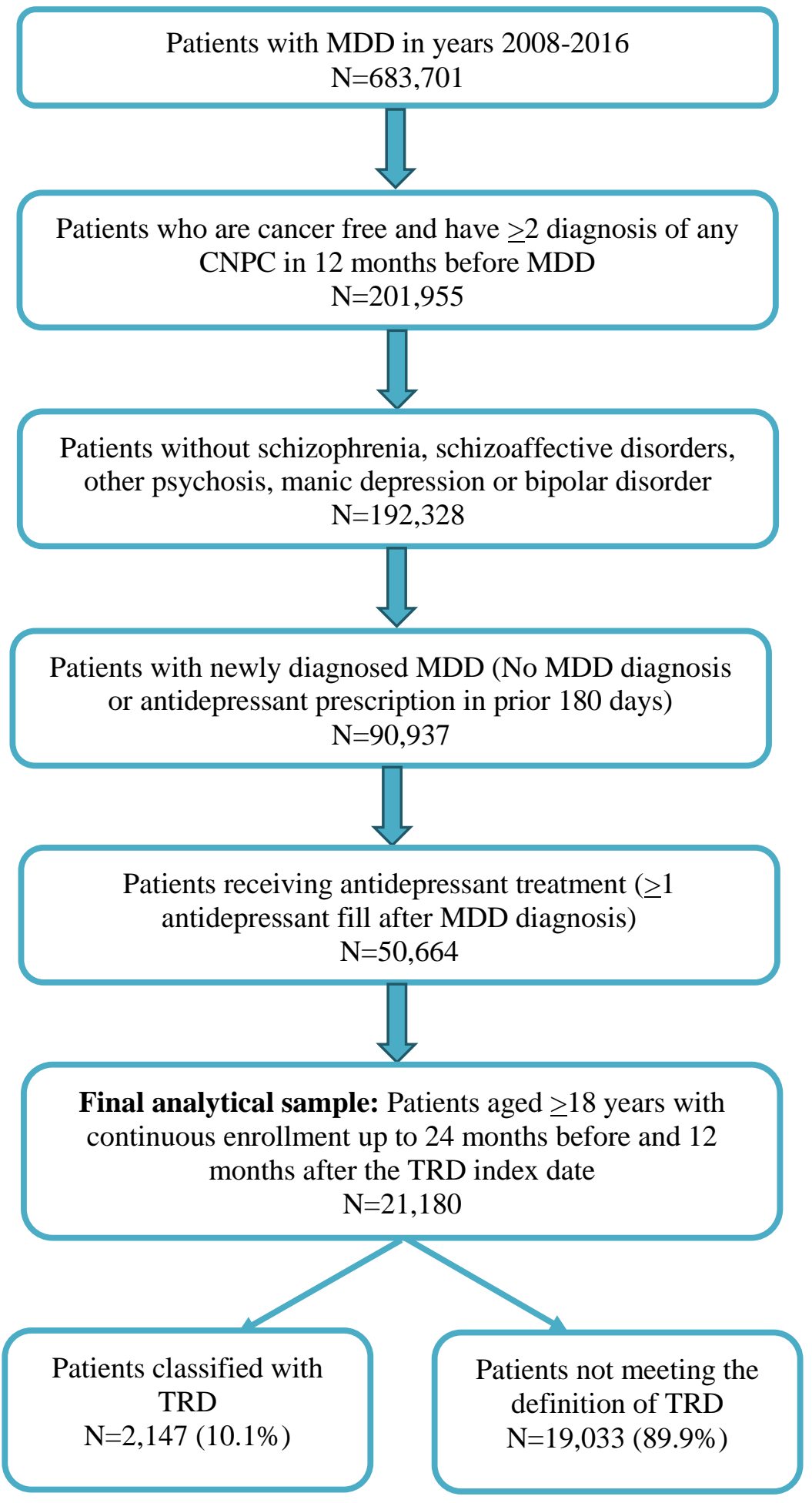




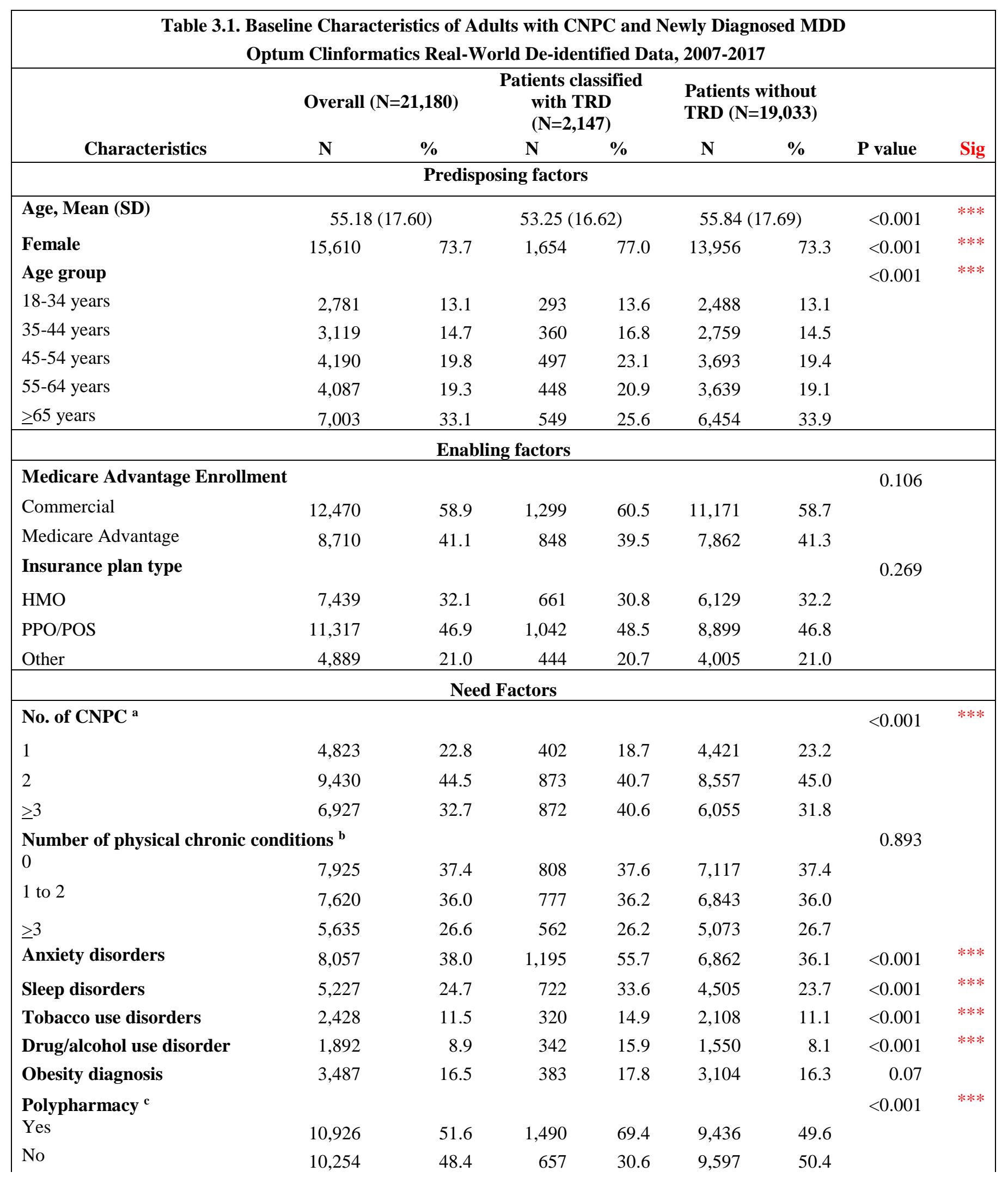




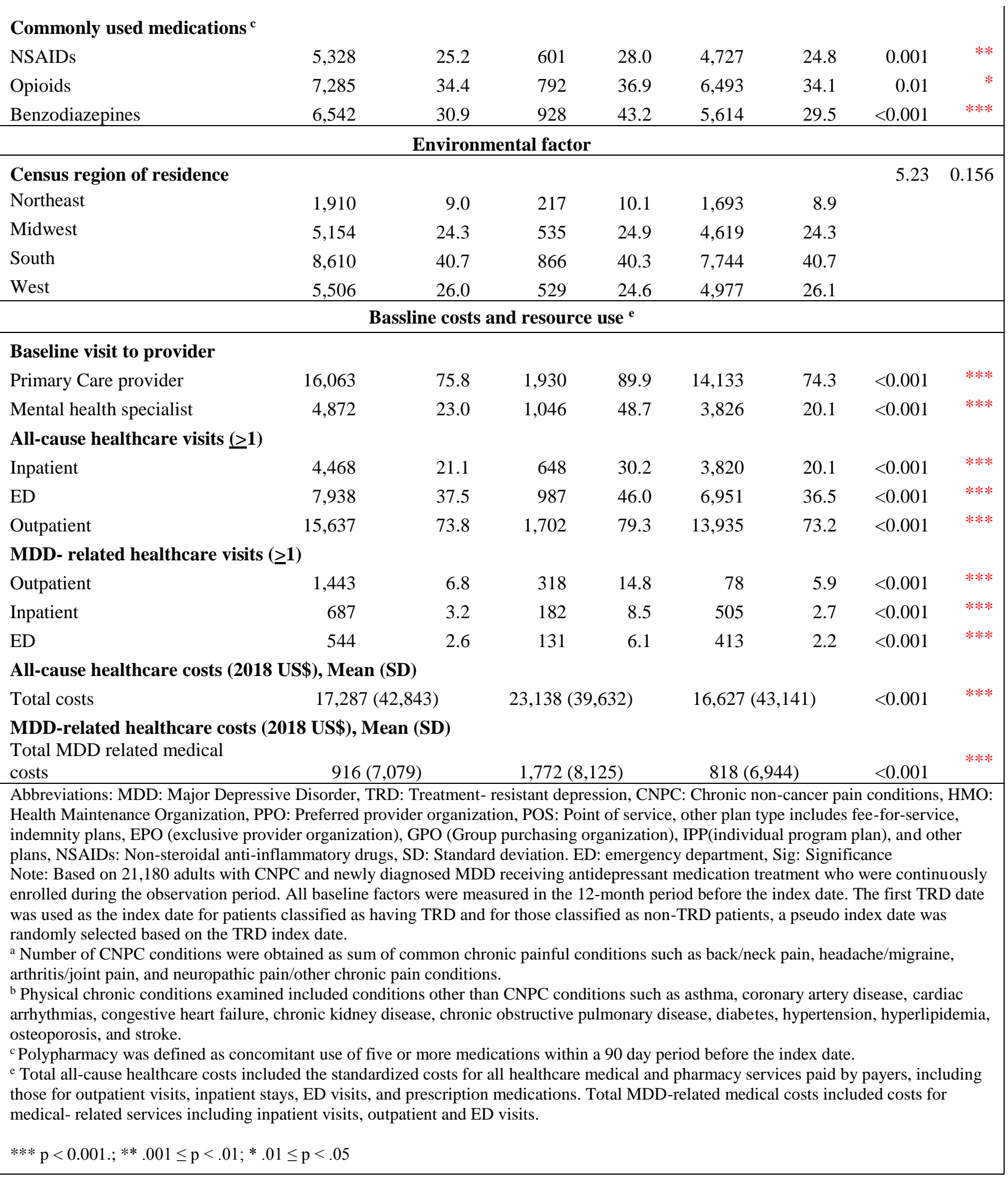




\begin{tabular}{|c|c|c|c|c|c|}
\hline \multicolumn{6}{|c|}{$\begin{array}{l}\text { Table 3.2. Mean Annual Adjusted and Unadjusted All-Cause and MDD-Related Costs } \\
\text { Among Adults with CNPC and Newly Diagnosed MDD by TRD status } \\
\text { Optum Clinformatics DataMart, 2007-2017 }\end{array}$} \\
\hline & $\begin{array}{l}\text { Patients } \\
\text { classified } \\
\text { withTRD }\end{array}$ & $\begin{array}{l}\text { Patients } \\
\text { without } \\
\text { TRD }\end{array}$ & $\begin{array}{c}\text { Unadjusted } \\
\text { Incremental costs }\end{array}$ & $\begin{array}{l}\text { Adjusted Incremental } \\
\text { costs, Model } 1^{\text {a }}\end{array}$ & $\begin{array}{l}\text { Adjusted Incremental } \\
\text { Costs,Model } 2^{\text {b }}\end{array}$ \\
\hline Outcomes & $\begin{array}{l}\text { Unadjusted } \\
\text { Mean (SD), } \\
\quad \$ \\
\end{array}$ & $\begin{array}{l}\text { Unadjuste } \\
\text { d Mean } \\
\text { (SD), \$ }\end{array}$ & $\begin{array}{c}\text { Mean } \$ \\
\text { (95\% CI); P value }\end{array}$ & $\begin{array}{c}\text { Mean \$ } \\
\text { (95\% CI); P value }\end{array}$ & $\begin{array}{c}\text { Mean } \$ \\
\text { (95\% CI); P value }\end{array}$ \\
\hline $\begin{array}{l}\text { All-cause he: } \\
\text { Total }\end{array}$ & $\begin{array}{l}\text { thcare costs }( \\
\quad 21,015 \\
(42,504)\end{array}$ & $\begin{array}{r}14,712 \\
(42,746)\end{array}$ & $\begin{array}{c}6303 \\
(4,396-8,209) ;<0.001\end{array}$ & $\begin{array}{c}3,388 \\
(3,353-3,419) ;<0.001\end{array}$ & $\begin{array}{c}2,025 \\
(2,015-2,056) ;<0.001\end{array}$ \\
\hline $\begin{array}{l}\text { Prescription } \\
\text { drug costs }\end{array}$ & $\begin{array}{c}6,286 \\
(10,014)\end{array}$ & $\begin{array}{l}3,913 \\
(9,050)\end{array}$ & $\begin{array}{c}2373 \\
(1,964-2,781) ;<0.001\end{array}$ & $\begin{array}{c}1,281 \\
(1,370-1,293) ;<0.001\end{array}$ & $\begin{array}{c}776 \\
(768-785) ;<0.001\end{array}$ \\
\hline Inpatient & $\begin{array}{c}7,603 \\
(34,608)\end{array}$ & $\begin{array}{c}5,667 \\
(34,969)\end{array}$ & $\begin{array}{c}1,936 \\
(377-3,495) ; 0.014\end{array}$ & $\begin{array}{c}1,329 \\
(1,313-1,346) ; 0.012\end{array}$ & $\begin{array}{c}894 \\
(883-907) ; 0.108\end{array}$ \\
\hline Outpatient & $\begin{array}{c}5,338 \\
(14,972)\end{array}$ & $\begin{array}{c}3,998 \\
(17,545)\end{array}$ & $\begin{array}{c}1,340 \\
(567-2,112) ;<0.001\end{array}$ & $\begin{array}{c}764 \\
(758-770) ; 0.008\end{array}$ & $\begin{array}{c}662 \\
(656-668) ; 0.004\end{array}$ \\
\hline ED & $\begin{array}{c}1,789 \\
(5,100)\end{array}$ & $\begin{array}{l}1,135 \\
(3,922)\end{array}$ & $\begin{array}{c}654 \\
(473-835) ;<0.001\end{array}$ & $\begin{array}{c}223 \\
(221-225) ; 0.002\end{array}$ & $\begin{array}{c}174 \\
(172-175) ; 0.011\end{array}$ \\
\hline \multicolumn{6}{|c|}{$\begin{array}{l}\text { MDD-related healthcare } \\
\text { costs (2018 US\$) }\end{array}$} \\
\hline Total & $\begin{array}{l}1,201 \\
(5853)\end{array}$ & $\begin{array}{c}471 \\
(3771)\end{array}$ & $\begin{array}{c}730 \\
(550-909) ;<0.001\end{array}$ & $\begin{array}{c}394 \\
(389-399) ;<0.001\end{array}$ & $\begin{array}{c}296 \\
(291-301) ;<0.001\end{array}$ \\
\hline Inpatient & $\begin{array}{c}543 \\
(4557)\end{array}$ & $\begin{array}{c}169 \\
(2890)\end{array}$ & $\begin{array}{c}374 \\
(236-513) ;<0.001\end{array}$ & $\begin{array}{c}276 \\
(272-280) ;<0.001\end{array}$ & $\begin{array}{c}228 \\
(224-231) ;<0.001\end{array}$ \\
\hline Outpatient & $\begin{array}{c}470 \\
(2,980)\end{array}$ & $\begin{array}{c}213 \\
(1,843)\end{array}$ & $\begin{array}{c}257 \\
(168-346) ;<0.001\end{array}$ & $\begin{array}{c}84 \\
(92-85) ;<0.001\end{array}$ & $\begin{array}{c}61 \\
(60-62) ;<.001\end{array}$ \\
\hline ED & $\begin{array}{c}188 \\
(1,178)\end{array}$ & $\begin{array}{c}89 \\
(869) \\
\end{array}$ & $\begin{array}{c}99 \\
(57-138) ;<0.001 \\
\end{array}$ & $\begin{array}{c}14 \\
(13-15) ; 0.001 \\
\end{array}$ & $\begin{array}{c}12 \\
(11-13) ; 0.004\end{array}$ \\
\hline \multicolumn{6}{|c|}{$\begin{array}{l}\text { Abbreviations: MDD: Major Depressive Disorder, TRD: Treatment- resistant depression, CNPC: Chronic non-cancer pain conditions, } \\
\text { SD: Standard deviation; ED: Emergency Department } \\
\text { Note: The sample includes all eligible adults with CNPC and newly diagnosed MDD who are treated with antidepressants and were } \\
\text { continuously enrolled throughout the study observation period (N=21,180) from the de-identified Optum Clinformatics DataMart. } \\
\text { All the costs were standardized to } 2018 \text { US dollar. Total all-cause healthcare costs included the standardized costs for all healthcare } \\
\text { medical and pharmacy services paid by payers, including those for outpatient visits, inpatient stays, ED visits, and prescription } \\
\text { medications. Total MDD-related medical costs included costs for medical- related services including inpatient visits, outpatient and ED } \\
\text { visits. Total MDD-related costs were identified based on primary/secondary diagnoses of MDD. } \\
\text { Unadjusted incremental costs and their corresponding P value and } 95 \% \text { CIs have been obtained using ordinary least square regressions. } \\
\text { a Incremental costs from Model } 1 \text { were measured using counterfactual recycled predictions performed using estimates obtained from a } \\
\text { 2-part GLM model with log link and gamma distribution which adjusted for baseline factors: predisposing factors (age, sex), enabling } \\
\text { factors (Medicare Advantage enrollment, type of insurance plan), need based factors (number of physical chronic conditions, number of } \\
\text { CNPC, presence of anxiety disorders, sleep disorders, obesity, and baseline medication use (polypharmacy, NSAIDs, opioid and } \\
\text { benzodiazepine use), and environmental factors (region). } \\
\text { b Incremental costs from Model } 2 \text { were measured using counterfactual recycled predictions performed using estimates obtained from a 2- } \\
\text { part GLM model with log link and gamma distribution which adjusted for all baseline factors mentioned in model } 1 \text { and healthcare costs. } \\
\text { a,b All P values and their confidence intervals were obtained using nonparametric bootstrap procedure (N=500) on the predicted values } \\
\text { obtained from recycled predictions. }\end{array}$} \\
\hline
\end{tabular}


Figure 3.2. Adjusted average annual healthcare resource utilization associated with TRD among adults with CNPC and newly diagnosed MDD

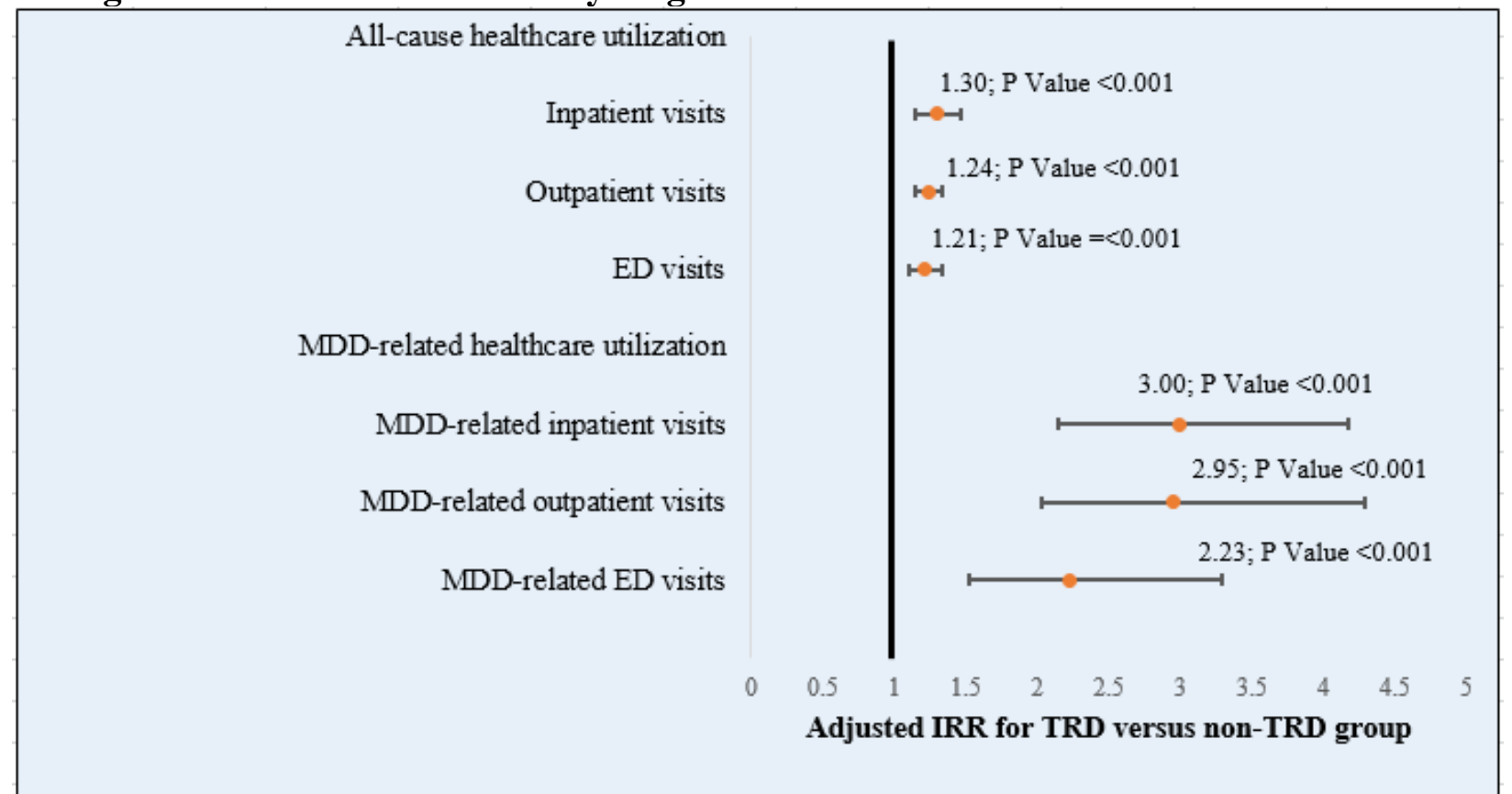

Note: All healthcare resource utilization was measured from the index date up to 12 months after the index date. The sample includes all eligible adults with CNPC and newly diagnosed MDD who are treated with antidepressants and were continuously enrolled throughout the study observation period $(\mathrm{N}=21,180)$ from the de-identified Optum Clinformatics DataMart.

Adjusted IRRs and their corresponding P values were obtained using a generalized linear model (GLM) with a negative binomial distribution (based on the results of the over dispersion test). The model adjusted for baseline predisposing factors (age, sex), enabling factors (Medicare Advantage enrollment, type of insurance plan), need based factors (number of physical chronic conditions, number of CNPC, presence of anxiety disorders, sleep disorders, obesity, and baseline medication use (polypharmacy, NSAIDs, opioid and benzodiazepine use), environmental factor (region), and baseline healthcare utilization. Abbreviations: MDD: Major Depressive Disorder, TRD: Treatment- resistant depression, CNPC: Chronic non-cancer pain conditions, SD: Standard deviation; ED: Emergency Department, IRR: Incidence Rate Ratio 


\begin{tabular}{|c|c|c|c|}
\hline \multicolumn{4}{|c|}{$\begin{array}{l}\text { Table 3.3. Post Linear Decomposition to Explain Contribution of Patient Factors on All-Cause Total } \\
\text { Healthcare Cost: Differences by TRD Status } \\
\text { Among Adults with CNPC and Newly Diagnosed MDD } \\
\text { Optum Clinformatics DataMart, 2007-2017 }\end{array}$} \\
\hline \multicolumn{4}{|c|}{ Explained Differences in Average Log-transformed All-Cause Total Healthcare Costs } \\
\hline & Pooled & & \\
\hline Characteristics & $\begin{array}{c}\text { Explained } \\
(\beta)\end{array}$ & SE; P Value & $\begin{array}{c}\% \\
\text { difference }^{d}\end{array}$ \\
\hline \multicolumn{4}{|l|}{ Predisposing factors } \\
\hline Age, Sex & 0.0127 & $0.0045 ; 0.006$ & $3.78 \%$ \\
\hline \multicolumn{4}{|l|}{ Enabling factors } \\
\hline Insurance plan type, Medicare Advantage Enrollment & 0.0003 & $0.0033 ; 0.938$ & $0.08 \%$ \\
\hline \multicolumn{4}{|l|}{ Need factors } \\
\hline No. of CNPC a & 0.0377 & $0.0051 ;<0.001$ & $11.24 \%$ \\
\hline Anxiety disorders, & 0.0274 & $0.0058 ;<0.001$ & $8.17 \%$ \\
\hline Tobacco use disorders. Drug/alcohol use disorder & 0.0369 & $0.0052 ;<0.001$ & $10.99 \%$ \\
\hline No. of physical chronic conditions ${ }^{b}$, Obesity diagnosis & -0.0021 & $0.0074 ; 0.779$ & $-0.63 \%$ \\
\hline Polypharmacy & 0.1870 & $0.0113 ;<0.001$ & $55.73 \%$ \\
\hline $\begin{array}{l}\text { Commonly used medications: NSAIDs use, Opioid } \\
\text { use, Benzodiazepine use }\end{array}$ & 0.0171 & $0.0045 ;<0.001$ & $5.09 \%$ \\
\hline \multicolumn{4}{|l|}{ External environment } \\
\hline Region & 0.02 & $0.0048 ;<0.001$ & $5.53 \%$ \\
\hline Total explained & 0.34 & & $46.02 \%$ \\
\hline \multicolumn{4}{|c|}{$\begin{array}{l}\text { Note: The sample includes all eligible adults with CNPC and newly diagnosed MDD who are treated with antidepressants and were } \\
\text { continuously enrolled throughout the study observation period }(\mathrm{N}=21,180) \text { from the de-identified Optum Clinformatics DataMart. } \\
\text { a Number of CNPC conditions were obtained as sum of common chronic painful conditions such as back/neck pain, } \\
\text { headache/migraine, arthritis/joint pain, and neuropathic pain/other chronic pain conditions. } \\
\text { b Physical chronic conditions examined included conditions other than CNPC conditions such as asthma, coronary artery disease, } \\
\text { cardiac arrhythmias, congestive heart failure, chronic kidney disease, chronic obstructive pulmonary disease, diabetes, hypertension, } \\
\text { hyperlipidemia, osteoporosis, and stroke. } \\
\text { c Polypharmacy was defined as concomitant use of five or more medications within a 90-day period before the index date. } \\
\text { All the costs were standardized to } 2018 \text { US dollar. Total all-cause healthcare costs included the standardized costs for all healthcare } \\
\text { medical and pharmacy services paid by payers, including those for outpatient visits, inpatient stays, ED visits, and prescription } \\
\text { medications. }\end{array}$} \\
\hline
\end{tabular}




\section{CHAPTER 4}

\section{Trajectories of Long-term Opioid Use: The Role of Treatment-Resistant Depression among Adults with Chronic Non-Cancer Pain Conditions and Newly Diagnosed Major Depressive Disorder}

\subsection{Abstract}

Background: Although treatment guidelines discourage long-term opioid therapy (LTOT) for patients with co-existing chronic non-cancer pain conditions (CNPC) and major depressive disorder (MDD), many patients receive LTOT in real-world practice settings. Further, treatmentresistant depression (TRD) may increase the risk of LTOT. We examined the trajectory of LTOT use before and after MDD diagnosis and the association of TRD with LTOT among patients with CNPC and newly diagnosed MDD.

Methods: Retrospective, longitudinal analysis of patients with CNPC and newly diagnosed MDD (N=21,599) using de-identified data from commercially insured adults (January 2007-June 2017) was conducted. TRD was identified using a claims-based algorithm and LTOT (defined as $>90$ consecutive days) of prescription opioids was measured every 180 days over a 3 -year period anchored to the date of MDD diagnosis. The adjusted relationship between TRD and LTOT was examined using generalized estimating equation models.

Results: In our study sample, $11.8 \%$ of adults with CNPC transitioned from MDD to TRD. During the study period, LTOT use declined from $15.6 \%$ in time 1 to $10.3 \%$ in time 6 (P <.001). Adults identified with TRD were more likely to receive LTOT, as compared to those without TRD [adjusted odds ratio (AOR): 1.54, 95\% CI: 1.68-2.04). However, LTOT in this group declined over time at a higher rate, than those without TRD $(\beta=-.033, \mathrm{SE}=0.012)$. 
Conclusion: Adults with TRD were more likely to receive LTOT at every time period over a 3year period than those without TRD, although LTOT use declined over time. Close monitoring of the risks of using LTOT among adults with CNPC and TRD is warranted.

\subsection{Introduction}

Given that many patients with chronic non-cancer pain conditions CNPC suffer from persistent pain, they often receive long-term opioid therapy (LTOT), ${ }^{81,126}$ commonly defined as continuous use of opioid beyond 90 days. ${ }^{126-128}$ Use of LTOT in patients with CNPC is controversial, ${ }^{33,34,53,129-131}$ with some believing that it may improve pain outcomes,${ }^{132}$ while others warning that the evidence is limited regarding the effectiveness of LTOT for different types of chronic pain such as neuropathic, nociceptive, and mixed pain types. ${ }^{133,134}$ Furthermore, LTOT often increases the risk of adverse events such as constipation, nausea, addiction, and opioid-induced hyperalgesia. ${ }^{134,135}$

In fact, clinical guidelines do not recommend use of LTOT among specific subgroups of patients, specifically those with co-occurring CNPC and major depressive disorder (MDD). ${ }^{55}$ Prevalence rates of MDD range from 28\% in population-based research to as high as $89 \%$ in orthopedic clinics. ${ }^{17,18}$ Prospective studies have reported that MDD makes chronic pain less responsive to opioids among adults with CNPC, ${ }^{55,136}$ further limiting the clinical benefits of LTOT in this subgroup. Despite lack of robust evidence on the long-term efficacy of opioids, many patients with CNPC and MDD receive LTOT in real-world practice settings. It has been reported that adults with MDD and CNPC are more likely to receive LTOT, as compared to those without MDD. ${ }^{56}$ Because pain is also a somatic symptom of depression, MDD can make chronic pain worse in adults with CNPC. This may lead to opioid prescribing at a much higher rate and longer duration among those with CNPC and MDD, than in patients who have CNPC 
without MDD. ${ }^{60,137,138}$ As MDD is harder to treat with antidepressants in the presence of chronic pain, ${ }^{11}$ LTOT may be prescribed at a higher rate for pain control to patients who do not respond to multiple antidepressant trials. ${ }^{55}$ Patients with MDD and co-occurring CNPC have a higher risk of developing treatment-resistant depression (TRD), ${ }^{20,22,24,29}$ commonly defined as failing multiple trials of antidepressants. ${ }^{17-20}$ TRD may increase the use of LTOT $^{55}$ as the neural patterns of activation that are associated with depression are also associated with pain, and LTOT may be prescribed to treat the complex combination of both pain and TRD. ${ }^{55,57}$

On the other hand, clinicians may be cautious in prescribing LTOT to patients with MDD who develop TRD because these patients may also have higher rates of substance use disorders, including opioid-use disorders. ${ }^{55,139}$ Therefore, it is plausible that LTOT use before and after MDD diagnosis may vary, and TRD may alter this trajectory. To date, no study has examined the trajectory of LTOT among adults with CNPC and newly diagnosed MDD, or the association of TRD with LTOT. TRD is now receiving increased attention because of its high clinical, economic and societal burden. ${ }^{20}$ The only retrospective study in the US that examined the relationship between TRD and LTOT focused on Veterans, and reported that LTOT is a risk factor for TRD. The study did not report on the association of TRD with LTOT. ${ }^{30}$

An understanding of the relationship between LTOT and TRD is important for several reasons. First, LTOT among adults with TRD may be deemed to be "high-risk" as both LTOT and TRD are independently associated with increased risk of substance use disorders, ${ }^{54,139}$ and adverse health outcomes ${ }^{20,22}$ Second, patients with TRD receiving LTOT comprise a patient population where the benefits of LTOT are questionable and vulnerability to risks of adverse events related to LTOT are very high; making it critical for healthcare providers to weigh the risks versus benefits of using LTOT in this group of patients. Furthermore, having an 
understanding of LTOT prescribing patterns among individuals with CNPC and newly diagnosed MDD is important to better inform clinical practice, payers, and policy makers in their surveillance and prevention efforts of controlling opioid- related misuse and abuse. To fill the existing knowledge gap and provide further insights on the real-world pattern of LTOT use, the current study sought to examine the trajectory of LTOT over a 3-year period in adults with CNCP and the association of TRD with LTOT among adults with CNPC and newly diagnosed MDD.

\subsection{Methods}

\section{Data Source}

The study used health claims from the de-identified 10\% sample drawn from the Optum Clinformatics ${ }^{\circledR}$ DataMart (Optum Clinformatics ${ }^{\circledR}$, Eden Prairie, MN, USA) ${ }^{67}$ from $1^{\text {st }}$ January 2007 through $30^{\text {th }}$ June 2017 . This geographically diverse database contains information on prescription drug claims [e.g. generic drug names, days' supply, prescription fill dates], medical claims (e.g. International Classification of Diseases, ninth/tenth revision [ICD-9/10] diagnoses, Current Procedural Terminology codes], and eligibility information [e.g. age, sex]. ${ }^{67}$

\section{Study Design}

We adopted a retrospective, observational, longitudinal cohort design, with repeated measurements of LTOT every 180-days for six time points (T1, T2, T3, T4, T5, and T6) over a 3-year observation period. Repeated measurements were anchored to the date of newly diagnosed MDD with pre-index (T1, T2) and post-index periods (T3, T4, T5, and T6). To allow sufficient time for follow-up newly diagnosed MDD was restricted to the period between January 1, 2008 to June 30, 2015. To ensure that the episode of MDD was newly diagnosed episode, we used a validated algorithm of including only those who did not have any MDD 
diagnosis or antidepressant prescription in 180 days prior to the MDD diagnosis date. ${ }^{78,79}$ A 12 month pre-index period was used for cohort identification and TRD was identified in the subsequent 12-month post-index period (T4 and T5) (Figure 4.1).

\section{Study Cohort}

The study cohort comprised commercially insured adults with CNPC and newly diagnosed MDD identified using ICD-9 codes (see Appendix 7.1 for classification codes). Adults with CNPC were required to have at least two healthcare encounters during the pre-index period. Chronic non-cancer pain conditions, identified using an extensive list of conditions for which pain was severe enough to call them as chronic pain conditions, ${ }^{80-82}$ was grouped into four broad categories encompassing the most common pain conditions (back/neck pain, headache/migraine, arthritis/joint pain, neuropathic/other chronic pain). ${ }^{127,140}$ Other inclusion/exclusion criteria were age $\geq 18$ years, at least one prescription of antidepressant following MDD diagnosis, no cancer diagnosis, and continuous enrollment throughout the study observation period. Additionally, similar to prior studies we excluded patients with any diagnosis of schizophrenia, schizoaffective disorder, other psychosis, manic depression or bipolar disorders. ${ }^{22,23}$ After applying the inclusion/ exclusion criteria, the final study sample consisted of 21,599 adults (Figure 4.2).

\section{Measures}

\section{Key Independent Variable: Treatment Resistant Depression}

Due to a lack of consensus on the definition of TRD, there are inconsistencies in the measures/algorithms used to identify TRD from a claims database. ${ }^{72,141,142}$ For the current study, adults with TRD were identified using a comprehensive claims-based staging algorithm that was developed using the General Hospital (MGH) clinical staging method. ${ }^{23,61}$ As per this algorithm, a patient with CNPC and MDD was classified as meeting the criteria for TRD if his/her MGH 
score exceeded three points. Patients were scored as follows: 1) one point was assigned for each adequate antidepressant trial (i.e., 2 or more fills of the same antidepressant); 2 ) half a point was assigned for each optimization strategy (included extended duration, i.e. at least 3 fills of the same antidepressant, an upward titration in dose, and augmentation with an atypical antipsychotic, mood stabilizer, thyroid hormone; ${ }^{23} 3$ ) Four points for patients using electroconvulsive therapy (i.e. those using ECT were directly considered to have TRD as ECT is often used for late-stage treatment for TRD. ${ }^{7}$ (See Appendix 7.10 for the list of augmentation medications and the number of patients meeting each of the MGH scoring criteria).

\section{Outcome: Long-term opioid therapy (LTOT)}

Opioids were identified using the therapeutic drug classification for opiate agonists (codeine, fentanyl, hydrocodone, hydromorphone, levorphanol, meperidine, morphine, oxycodone, oxymorphone, and pentazocine) from the dataset. ${ }^{30}$ Following the algorithms used in published literature, LTOT was defined as receiving greater than 90 days' supply of opioids with less than a 30-day gap between two fills within a 180-day period. ${ }^{30,127,128,140,143} \mathrm{~A}$ threshold of $>90$ days was chosen because it corresponds to usually 4 or more prescriptions and it is unlikely for an individual to receive opioids for $>90$ days for acute pain conditions. Additionally, this threshold represents an important point in the treatment process where clinicians would want to be cautious of the clinical risk of continuing opioid treatment, thereby making it a reasonable threshold for risk analysis. ${ }^{128,140}$ We explored the effect of TRD on LTOT using a sensitivity analysis by defining LTOT using a 120-day period of continuous opioid supply with a 30-day permissible gap..$^{56}$ In the current study, we used LTOT as defined by duration of opioid use as our outcome variable and not dose of opioids because previous studies have highlighted that new onset of MDD and TRD is associated with longer duration of opioids and not dose. ${ }^{30,137,144}$

\section{Other Independent Variables}


Selection of other explanatory variables (time invariant and time-varying) was guided by the expanded Symptom Management Model. ${ }^{65,66}$ As per the proposed framework, presence of TRD, which is mainly non-response to antidepressant treatment trials, was considered as a symptom experience which can influence the outcome of LTOT. As per the model, the above dimensions can be influenced by the following three domains: (1) the personal domain, which comprised of demographic and socio-economic factors (e.g. age, sex, insurance plan type, Medicare advantage enrollment), (2) the health and illness domain (e.g. chronic physical conditions, obesity, sleep disorders, substance use disorders, polypharmacy, benzodiazepine use, and use of pain medication such as NSAIDs); (3) the environmental domain (e.g. geographical region, index year). Our study included both time varying and time invariant factors. Substance-use disorders, polypharmacy, NSAIDs and benzodiazepine use were measured during each time interval from T1 to T6. All other variables were measured during the baseline (pre-index period).

\section{Statistical Analyses}

Differences in baseline sample characteristics among adults by LTOT status in the preindex period and TRD status in the post-index period (T4 and T5) were compared using chisquare tests for categorical variables and t-tests for continuous variables (e.g. age). Considering that LTOT was measured repeatedly every 180-days during the pre- and post-index period, with each individual having six observations, these six observations were not independent. As standard regression techniques cannot be applied to correlated observations, we used unadjusted and adjusted generalized estimating equations (GEE). ${ }^{145}$ The GEE accounts for correlated error terms due to repeated measures from the same individual and is fairly robust to choice of correlation matrix, particularly with large sample sizes. In the current study, population-averaged 
GEE with binary distribution was used. A population averaged model provides group effect, in contrast to subject-specific model which includes a 'unique' effect for each individual. ${ }^{146}$

We also analyzed the interaction between TRD and time to elucidate the impact of TRD on the trajectory of use of LTOT over time. Unadjusted and adjusted Odds Ratios and their corresponding $95 \%$ confidence intervals have been reported. The adjusted GEE model controlled for time, personal, health and illness, and environmental factors. All data management and analyses were performed with SAS 9.4 (SAS Institute Inc., Cary, NC) and Stata 14 (StataCorp LLC, College Station, TX).

\subsection{Results}

\section{Sample Characteristics}

Among 21,599 adults with CNPC and newly diagnosed MDD, 2550 (11.80\%) met the criteria for TRD within one year of MDD diagnosis, with a mean time of 8 months. Adults with CNPC and newly diagnosed MDD were mostly females (73.6\%) with a mean age of 55.2 years. The majority of adults in our study had arthritis/joint pain (91\%), followed by back/neck pain (71\%). About $16 \%$ of adults were on LTOT during the pre-index (baseline) period. (Table 4.1).

\section{Sample Characteristics by TRD}

TRD patients were mostly younger with a mean age of 53.7 years, as compared to those without TRD (mean age $=55.43$ years). There were significant differences in personal factors and health and illness -related factors between adults with and without TRD. For example, a greater

percentage of adults with TRD had $\geq 3$ CNPC (41.4\% vs. $32.2 \%)$, had co-existing sleep disorders (29.9\% vs. $22.0 \%)$, anxiety disorders (36.9\% vs. $26.5 \%)$, and used polypharmacy (59.1\% vs. 43.4\%), as compared to CNPC patients without TRD (all $P<.001$ ) (Appendix 7.11).

\section{Sample Characteristics by use of LTOT}


The baseline sample characteristics among those using LTOT and not using LTOT in the pre-index period differed significantly across all domains, including personal, environmental, and health and illness factors (All $P<0.01)$ (Table 4.1). For example, a higher percentage of adults on LTOT were males ( $28.3 \%$ vs. $16.1 \%)$, had $\geq 3$ CNPC (58.5\% vs. $28.2 \%)$, and had $\geq 3$ physical chronic conditions (35.0\% vs. $26.3 \%)$.

\section{Trajectory of LTOT over 3-year study period}

In our sample, LTOT use declined from $15.7 \%$ in T1 to $10.3 \%$ in T6 and the decline was significant (Figure 4.3). In unadjusted GEE models, when time was included as the only

covariate, the time co-efficient was found to be negative $(\hat{\beta}=-.055, \mathrm{SE}=.003)$, suggesting that LTOT use decreased over time. For example, LTOT use was less likely at T6 (OR = 0.76, 95\% $\mathrm{CI}=0.73-0.79)$, compared to the pre-index (T2) period (Table 4.2). When examined by TRD status, a significantly higher percentage of adults with TRD used LTOT $(P<.001)$ as compared to non-TRD patients at all time-points (Figure 4.3). Those with TRD were more likely to receive LTOT $(\mathrm{OR}=1.76 ; 95 \% \mathrm{CI}=1.62-1.91)$, after adjustment for time. Over time, the difference in LTOT rates among TRD and non-TRD patients narrowed (T1: 9.7 percentage point difference; T6: 5.0 percentage point difference). The interaction term between time and TRD status was negative $(\hat{\beta}-.022, \mathrm{SE}=.01)$, suggesting that TRD patients had a greater decline in LTOT, compared to non-TRD patients (Table 4.2).

In fully-adjusted models (after adjusting for TRD, time-interaction with TRD, personal, environmental, and health and illness factors), adults with TRD were 1.5 times as likely to use LTOT, as compared to adults without TRD (AOR=1.55, 95\% CI=1.39-1.71, $P$ value $<0.001)$. The interaction term between time and TRD status remained negative $(\hat{\beta}-.033, \mathrm{SE}=0.012)$ and 
statistically significant $(P$ Value $=0.006)$. The sensitivity analyses, where LTOT was defined as

$>120$ days' supply of opioids, showed similar results (Appendix 7.12 and 7.13).

Other factors associated with LTOT included: male sex, younger age (35-64 years), greater number of CNPC and physical chronic health conditions, comorbid anxiety disorders, polypharmacy, any use of NSAIDs, and benzodiazepines (Table 4.2). Furthermore, adults diagnosed with MDD in years after 2010, had lower odds of LTOT use, as compared to those diagnosed in years 2008-2010 (years 2011-2013: AOR: 0.86, 95\% CI: 0.79-0.94; years 20142015: AOR: 0.66, 95\% CI: 0.61-0.72).

\subsection{Discussion}

To the best of our knowledge, this is the first real-world study that examined the trajectory of LTOT among adults with CNPC and newly-diagnosed MDD, and the association of TRD with LTOT. We found an average LTOT rate of $16 \%$, which is in line with a previous study that reported a prevalence of $12 \%$ to $30 \%$ LTOT use among adults with CNPC and mood disorders. ${ }^{140}$ Overall, we found that adults who transitioned from MDD to TRD were significantly more likely to receive LTOT, as compared to adults who did not transition to TRD. This difference remained statistically significant at all time points. Even after adjusting for time and other patient factors, adults with TRD were more likely to receive LTOT (AOR=1.54, $P$ value $<0.001$ ), compared to those without TRD. Although it was not possible to ascertain reasons for higher LTOT use among TRD patients, there are some possible explanations based on what is known about TRD and chronic pain. It is well known that adults with TRD report greater severity of depression, physical symptoms, higher levels of pain, and a higher number of

CNPC, as compared to those with MDD without TRD. ${ }^{22,24,26} 11,147$ It is plausible that those with 
TRD have more severe pain which may not be responsive to first line pain therapies and opioids may be prescribed to these patients to treat an undifferentiated state of mental and physical pain.

While TRD is associated with LTOT, we cannot rule out the possibility that LTOT may worsen depression symptoms over time,${ }^{55}$ which may lead to TRD. ${ }^{30}$ In fact, to date, only one retrospective cohort study in the United States (US) has reported increased risk of new onset TRD due to longer duration of opioid use among Veterans. ${ }^{30} \mathrm{~A}$ recent study has also reported that mu opioid receptor agonists may worsen depression symptoms thereby increasing the risk of TRD. ${ }^{30}$ On the other hand, emerging evidence from clinical trials suggests that antagonists of the kappa opioid receptor, such as buprenorphine, may improve depression symptoms, ${ }^{148,149}$ reducing the risk of TRD. ${ }^{149}$ Therefore, future real-world studies are needed to explore the bidirectional relationships between LTOT and TRD, and the role of antagonists of the kappa opioid receptors for managing both pain and TRD.

A noteworthy finding was the general decline in LTOT over the 3-year observation period among CNPC patients newly diagnosed with MDD in recent years. Additionally, adults diagnosed with MDD in recent years (2011-2015) were less likely to use LTOT, as compared to those diagnosed in years 2008-2010. We also observed a greater decline in LTOT use among patients with TRD, as compared to those without TRD. Although comparable study findings do not exist in the currently published literature, the discontinuation of LTOT over time has been documented to be more pronounced among patients with substance-use disorders who are deemed to be a high risk group. ${ }^{150} \mathrm{We}$ speculate clinician initiated discontinuation may be higher among adults with TRD, as they are recognized as a high-risk group, given their greater severity of depression symptoms, higher risk of suicidal ideation and substance-use disorders $55,56,138$. Therefore clinicians may decide to taper the use of LTOT because of lack of pain relief, opioid- 
related side effects, and adverse events including opioid dependence and misuse. ${ }^{151}$ Such trends are encouraging and may be due to increased attention to opioid abuse and awareness of their risks in the past few years. ${ }^{152}$

However, the consistently higher LTOT use among adults with TRD over all time periods suggests the need to closely monitor CNPC patients with MDD and TRD. Considering the lack of robust evidence on the effectiveness of opioids for CNPC, ${ }^{33,34,53,129-131}$ and clinical guidelines recommending against LTOT for chronic pain and MDD,${ }^{55}$ future studies are needed to identify the reasons for high rates of LTOT among TRD patients. Providers may need to weigh the risks versus benefits of using LTOT in this patient population.

Additionally, our results also indicated that patient-level risk factors for LTOT identified from our study, including male sex, age, higher number of CNPC, higher number of physical health conditions, presence of anxiety disorders and, benzodiazepine use have also been identified by other studies as risk factors for clinically recognized opioid dependence and abuse among adults with CNPC. ${ }^{55,128,153}$ Providers need to assess risk factors while deciding appropriate pain management strategies for patients with CNPC and MDD. Providing collaborative multidisciplinary care for patients with CNPC, MDD and other chronic conditions in an integrated setting (e.g. patient centered medical homes) can optimize interactions between primary care providers and mental health specialists, improving patient outcomes. ${ }^{154} 155$ Additionally, behavioral interventions such as mindfulness-based or cognitive behavioral therapy can be effective alternative strategies to manage patients with co-occurring chronic pain, depression and other mental and physical health conditions. ${ }^{156}$

\subsection{Limitations}


The study has several strengths, including availability of a large cohort of patients with CNPC and MDD treated for depression in a real-world setting, and utilized a longitudinal study design to track individuals over time, enabling assessment of temporal relationships. Our study used a comprehensive algorithm that encompasses the four main strategies to overcome lack of response, including optimization, switching, combination, and augmentation ${ }^{62,106}$ to identify adults with TRD. To ensure robustness of study findings, the current study also tested the impact of TRD on LTOT by varying the definitions of the study outcome. However, several limitations should also be noted. First, TRD exists along a clinical continuum and there is a lack of consensus on its definition. Although, the current study used a comprehensive claims-based algorithm to define TRD mainly through pharmacy claims, a study relying on claims-based information does not give comparable level of clinical information such as persistence of depression symptoms, and tolerance to antidepressants. Further, lack of information on severity of MDD, pain level, socio-economic status, and race/ethnicity may lead to unobserved selection bias. It is plausible that our algorithm for identifying newly diagnosed episode of MDD (a lookback period of 180 days) may not truly capture all incident MDD patients. Furthermore, prescription drug claims provide information only on filled prescriptions and not actual use. Lastly, no direct inference should be made about the prevalence of TRD among adults with CNPC and newly diagnosed MDD, given the specific inclusion /exclusion criteria of the study. Although, our observed prevalence of $11.8 \%$ is consistent with some previously reported estimates of TRD among adults with MDD obtained from other claims-based studies (6\% to $15 \%)^{22,29,44,46}$

\subsection{Conclusion}


The present study demonstrated that LTOT declined over time. Although the decline in LTOT was greater in patients with TRD, LTOT rates were consistently higher among TRD patients, as compared to those without TRD. Considering that patients with CNCP and MDD who transition into TRD and continue to use LTOT are a high-risk group with substantial disease burden, multidisciplinary and targeted behavioral approaches along with integrated care, may be needed to co-manage CNCP and MDD. 
Figure 4.1. Schematic of the study design

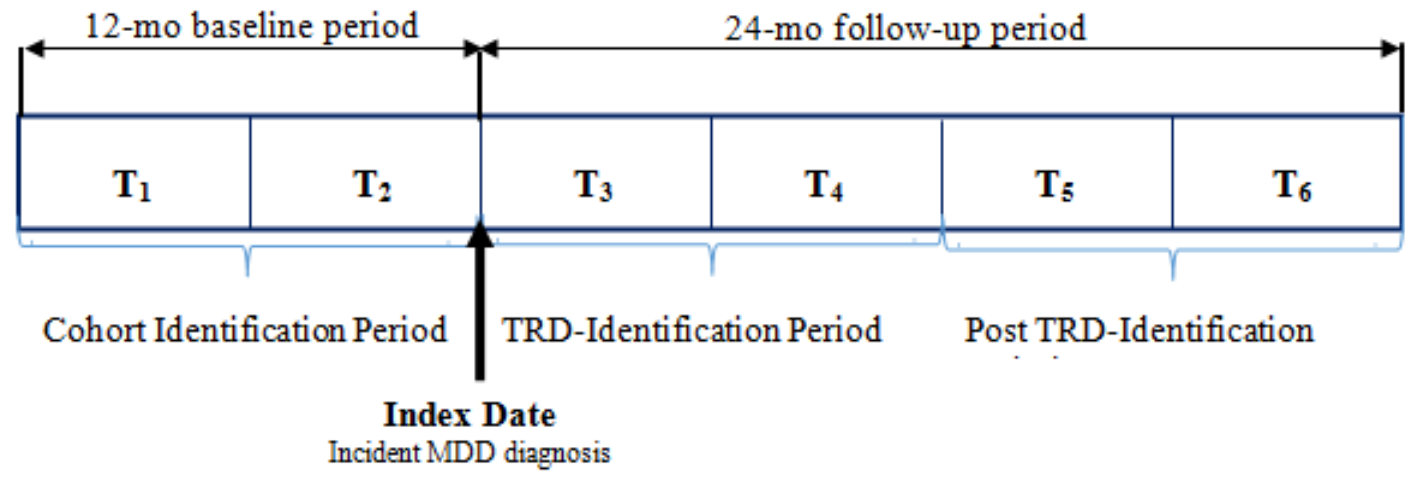

Each individual was observed for 36 months with a 12-month baseline period, (cohort identification period where adults with CNPC and newly diagnosed MDD were identified), 12- month TRD identification period and 12-month post TRD -identification period (follow-up periods). LTOT was measured repeatedly every 180 days during the baseline and follow-up periods, yielding a total of 6 repeated measures for each individual.

Abbreviations: MDD; Major depressive disorder; CNPC: Chronic non-cancer pain conditions; TRD: Treatment-resistant depression; LTOT: Long-term opioid therapy

Figure 4.2. Schematic presentation of study sample selection

\begin{tabular}{|c|}
\hline Attrition Flowchart \\
\hline Total cases of MDD from January 1,2008 through December 31,2016 \\
$(\mathbf{N}=\mathbf{5 1 8 , 3 9 6})$
\end{tabular}


Table 4.1. Overall Baseline Sample Characteristics and Characteristics by use of LTOT among Adults with CNPC and Newly Diagnosed MDD (measured during pre-index period)

Optum Clinformatics Real-World De-identified Data, 2007-2017

\begin{tabular}{|c|c|c|c|c|c|c|c|c|}
\hline \multirow[b]{2}{*}{ Characteristics } & \multicolumn{2}{|c|}{$\begin{array}{l}\text { Overall Sample } \\
\quad(\mathrm{N}=21,599)\end{array}$} & \multicolumn{2}{|c|}{$\begin{array}{l}\text { Use of LTOT } \\
(\mathrm{N}=3,561)\end{array}$} & \multicolumn{2}{|c|}{$\begin{array}{l}\text { No use of LTOT } \\
(\mathbf{1 8 , 0 3 8 )}\end{array}$} & \multirow[b]{2}{*}{$\underset{\text { value }}{\mathbf{P}}$} & \multirow[b]{2}{*}{ Sig } \\
\hline & $\mathbf{N}$ & $\begin{array}{c}\text { Percent } \\
(\%)\end{array}$ & $\mathbf{N}$ & $\begin{array}{c}\text { Percent } \\
(\%)\end{array}$ & $\mathbf{N}$ & $\begin{array}{c}\text { Percent } \\
(\%)\end{array}$ & & \\
\hline \multicolumn{9}{|c|}{ Personal factors } \\
\hline Age, Mean (SD) & \multicolumn{2}{|c|}{$55.24(17.51)$} & \multicolumn{2}{|c|}{$59.65(14.30)$} & \multicolumn{2}{|c|}{$54.36(17.94)$} & $<0.001$ & **** \\
\hline Female & 15,902 & 73.6 & 2,553 & 71.7 & 13,349 & 83.9 & 0.004 & $* *$ \\
\hline Age group & & & & & & & $<0.001$ & $* * *$ \\
\hline $18-34$ years & 2,837 & 13.1 & 151 & 4.2 & 2,686 & 14.9 & & \\
\hline $35-44$ years & 3,342 & 15.5 & 381 & 10.7 & 2,961 & 16.4 & & \\
\hline $45-54$ years & 4,354 & 20.2 & 777 & 21.8 & 3,577 & 19.8 & & \\
\hline $55-64$ years & 4,112 & 19.0 & 933 & 26.2 & 3,179 & 17.6 & & \\
\hline$\geq 65$ years & 6,954 & 32.2 & 1,319 & 37 & 5,635 & 31.2 & & \\
\hline Medicare Advantage Enrollment & & & & & & & $<0.001$ & $* * *$ \\
\hline Commercial & 13,119 & 60.7 & 2,145 & 60.2 & 11,703 & 64.9 & & \\
\hline Medicare Advantage & 8,480 & 39.3 & 1,416 & 39.8 & 6,335 & 35.1 & & \\
\hline Insurance plan type & & & & & & & $<0.001$ & $* * *$ \\
\hline HMO & 6,893 & 31.9 & 1,450 & 40.7 & 5,443 & 30.2 & & \\
\hline $\mathrm{PPO} / \mathrm{POS}$ & 10,346 & 47.9 & 1,192 & 33.5 & 9,154 & 50.7 & & \\
\hline Other & 4,360 & 20.2 & 919 & 25.8 & 3,441 & 19.1 & & \\
\hline \multicolumn{9}{|c|}{ Environmental factors } \\
\hline Census region of residence & & & & & & & $<0.001$ & $* * *$ \\
\hline Northeast & 2,001 & 7.1 & 254 & 10.5 & 1,747 & 9.7 & & \\
\hline Midwest & 5,363 & 19.8 & 705 & 25.4 & 4,658 & 25.8 & & \\
\hline South & 8,684 & 45.5 & 1,621 & 39.3 & 7,063 & 39.2 & & \\
\hline West & 5,551 & 27.5 & 981 & 24.8 & 4,570 & 25.3 & & \\
\hline \multicolumn{9}{|l|}{ Index Year } \\
\hline Years 2008-2010 & 6,271 & 29 & 925 & 26.0 & 5,346 & 29.6 & $<0.001$ & $* * *$ \\
\hline Years 2011-2013 & 7,105 & 32.9 & 1,169 & 32.8 & 5,936 & 32.9 & & \\
\hline Years 2014-2015 & 8,223 & 38.1 & 1,467 & 41.2 & 6,756 & 37.5 & & \\
\hline \multicolumn{9}{|c|}{ Health and illness related factors } \\
\hline \multicolumn{9}{|l|}{ Type of CNPC } \\
\hline Back/Neck Pain (yes) & 15,368 & 71.2 & 3,071 & 86.2 & 12,297 & 68.2 & $<0.001$ & $* * *$ \\
\hline Arthritis/Joint Pain(yes) & 19,728 & 91.3 & 3,364 & 94.5 & 16,364 & 90.7 & $<0.001$ & $* * *$ \\
\hline Headache/Migraine (yes) & 7,135 & 33 & 1,255 & 35.2 & 5,880 & 32.6 & 0.002 & $* *$ \\
\hline $\begin{array}{l}\text { Neuropathic/other chronic pain } \\
\text { (yes) }\end{array}$ & 4,774 & 22.1 & 1,904 & 53.5 & 2,870 & 15.9 & $<0.001$ & $* * *$ \\
\hline No. of CNPC a & & & & & & & $<0.001$ & $* * *$ \\
\hline 1 & 4,813 & 22.3 & 287 & 8.1 & 4,526 & 25.1 & & \\
\hline 2 & 9,604 & 44.5 & 1,179 & 33.1 & 8,425 & 46.7 & & \\
\hline$\geq 3$ & 7,182 & 33.3 & 2,095 & 58.8 & 5,087 & 28.2 & & \\
\hline \multicolumn{7}{|c|}{ Number of physical chronic conditions b } & $<0.001$ & $* * *$ \\
\hline 0 & 8,947 & 37.4 & 902 & 25.3 & 8,045 & 44.6 & & \\
\hline 1 to 2 & 7,566 & 36.0 & 1,446 & 40.6 & 6,120 & 33.9 & & \\
\hline$\geq 3$ & 5,086 & 26.6 & 1,213 & 34.1 & 3,873 & 21.5 & & \\
\hline
\end{tabular}




\begin{tabular}{|c|c|c|c|c|c|c|c|c|}
\hline Anxiety disorders(yes) & 5,987 & 27.7 & 1,246 & 35.0 & 4,741 & 26.3 & $<0.001$ & $* * *$ \\
\hline Sleep disorders(yes) & 4,957 & 23.0 & 1,073 & 30.1 & 3,884 & 21.5 & $<0.001$ & $* * *$ \\
\hline Substance use disorders (yes) & 1,308 & 6.1 & 480 & 13.5 & 828 & 4.6 & $<0.001$ & $* * *$ \\
\hline Obesity diagnosis (yes) & 3,366 & 15.6 & 713 & 20.0 & 2,653 & 14.7 & $<0.001$ & $* * *$ \\
\hline Polypharmacy $^{\mathrm{c}}$ & & & & & & & $<0.001$ & $* * *$ \\
\hline Yes & 9,768 & 45.2 & 2,680 & 75.3 & 7,088 & 39.3 & & \\
\hline No & 11,831 & 54.8 & 1,043 & 40.9 & 10,788 & 56.6 & & \\
\hline \multicolumn{9}{|l|}{ Commonly used medications ${ }^{c}$} \\
\hline NSAIDS use & 5,576 & 25.8 & 1,224 & 34.4 & 4,352 & 24.1 & $<0.001$ & $* * *$ \\
\hline Benzodiazepine use & 6,039 & 28.0 & 1,562 & 43.9 & 4,477 & 24.8 & $<0.001$ & **** \\
\hline \multicolumn{9}{|c|}{$\begin{array}{l}\text { Abbreviations: MDD: Major Depressive Disorder, TRD: Treatment- resistant depression, CNPC: Chronic non-cancer pain } \\
\text { conditions, HMO: Health Maintenance Organization, PPO: Preferred provider organization, POS: Point of service, other plan } \\
\text { type includes fee-for-service, indemnity plans, EPO (exclusive provider organization), GPO (Group purchasing organization), } \\
\text { IPP(individual program plan), and other plans, NSAID: Non-steroidal anti-inflammatory drugs, SD: Standard deviation, Sig: } \\
\text { Significance } \\
\text { Note: Based on } 21,599 \text { adults with CNPC and newly diagnosed MDD receiving antidepressant medication treatment who } \\
\text { were continuously enrolled during the observation period. LTOT as well as all baseline sample characteristics factors were } \\
\text { measured during the pre-index period (t2). } \\
\text { Long-term opioid therapy (LTOT) was defined as at least a }>90 \text { days of continuous supply of prescription opioids within a } \\
180 \text { day period } \\
\text { a Number of CNPC conditions were obtained as sum of common chronic painful conditions such as back/neck pain, } \\
\text { headache/migraine, arthritis/joint pain, and neuropathic pain/other chronic pain conditions. } \\
\text { b Physical chronic conditions examined included conditions other than CNPC conditions such as asthma, coronary artery } \\
\text { disease, cardiac arrhythmias, congestive heart failure, chronic kidney disease, chronic obstructive pulmonary disease, diabetes, } \\
\text { hypertension, hyperlipidemia, osteoporosis, and stroke. } \\
\text { c Polypharmacy was defined as concomitant use of five or more medications within a } 90 \text { day period before the index date. } \\
*_{* * *} \text { p }<0.001 . ; * * .001 \leq \mathrm{p}<.01 ; * .01 \leq \mathrm{p}<.05\end{array}$} \\
\hline
\end{tabular}


Figure 4.3. Percentage of LTOT use over time for all adults and by TRD status among adults with CNPC and newly diagnosed MDD

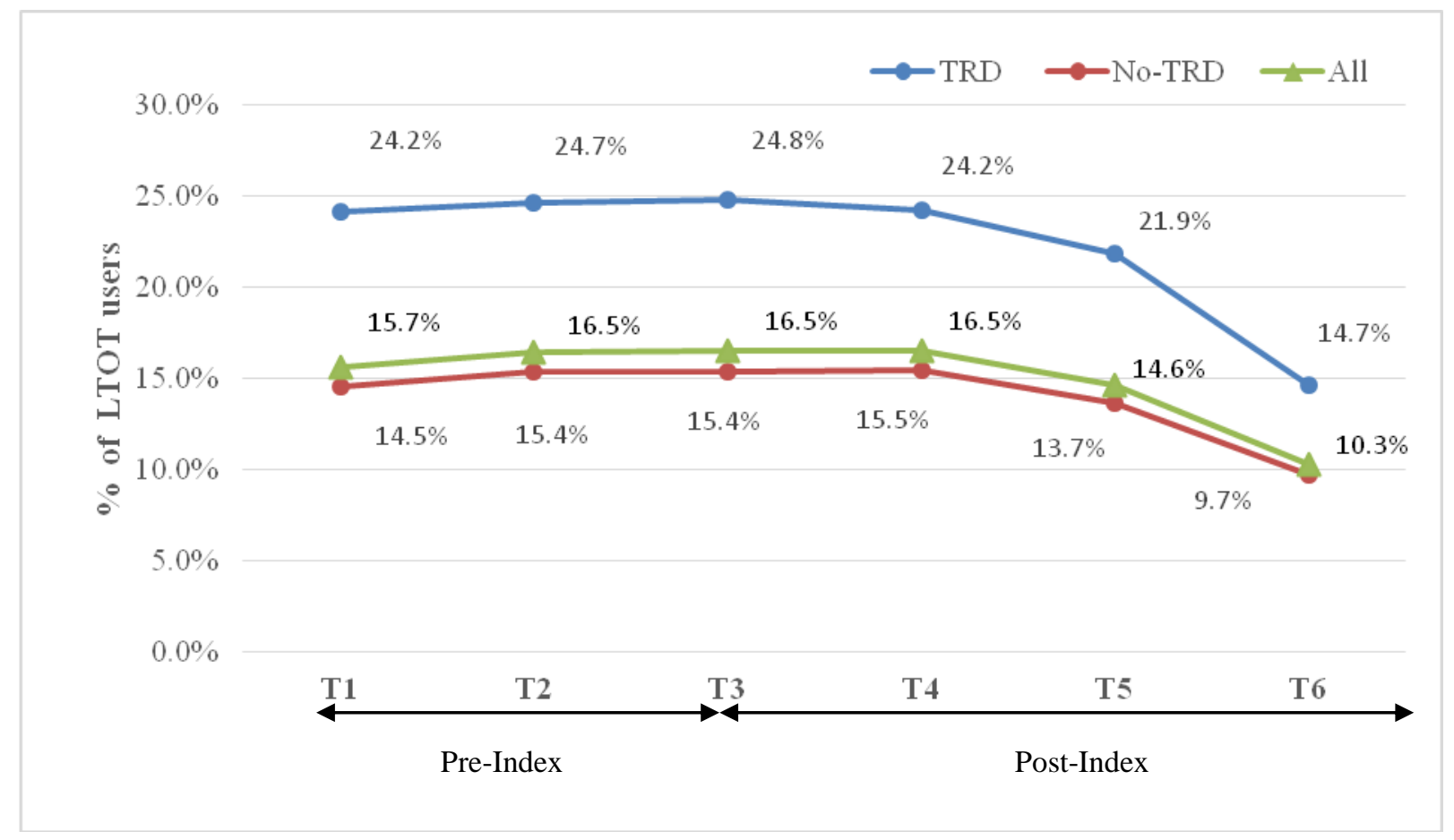

Abbreviations: MDD: Major Depressive Disorder, TRD: Treatment- resistant depression, CNPC: Chronic non-cancer pain conditions, LTOT: Long-term opioid therapy

LTOT was defined as at least a $>90$ days of continuous supply of prescription opioids within a 180-day period.

$P$ values for percentage of LTOT use over time for all adults, adults with TRD and without TRD were $<0.001$

$P$ values were obtained from unadjusted Generalized estimating equation models. 


\begin{tabular}{|c|c|c|c|c|c|}
\hline \multicolumn{6}{|c|}{$\begin{array}{l}\text { Table 4.2: Unadjusted and Adjusted Odds Ratio and 95\% Confidence Interval } \\
\text { from Generalized Estimating Equation (GEE) of TRD on Long -term opioid } \\
\text { therapy among } \\
\text { Adults with CNPC and Newly Diagnosed MDD } \\
\text { Optum Clinformatics Real-World De-identified Data, 2007-2017 }\end{array}$} \\
\hline Characteristics & $\begin{array}{r}\text { Parameter } \\
\text { estimate }(\beta)\end{array}$ & SE & OR & $95 \% \mathrm{CI}$ & $\begin{array}{c}\mathbf{P} \\
\text { Value }\end{array}$ \\
\hline \multicolumn{6}{|c|}{ Model 1: Unadjusted association between time and LTOT } \\
\hline Time & -0.055 & 0.003 & & & $<0.001$ \\
\hline \multicolumn{6}{|c|}{ Model 2: Unadjusted Association of time and TRD with LTOT } \\
\hline \multicolumn{6}{|l|}{ TRD (Ref: No TRD) } \\
\hline Yes & 0.56 & 0.043 & 1.76 & {$[1.62,1.91]$} & $<0.001$ \\
\hline Time & -0.056 & 0.003 & - & - & \\
\hline \multicolumn{6}{|c|}{$\begin{array}{c}\text { Model 2: Unadjusted Association of time, TRD, and interaction of time and TRD } \\
\text { with LTOT }\end{array}$} \\
\hline \multicolumn{6}{|c|}{ TRD (Ref: No TRD) } \\
\hline Yes & 0.62 & 0.050 & 1.85 & {$[1.68,2.04]$} & $<0.001$ \\
\hline Time & -0.052 & 0.004 & - & - & $<0.001$ \\
\hline TRD*Time & -0.022 & 0.020 & - & - & 0.037 \\
\hline \multicolumn{6}{|c|}{$\begin{array}{c}\text { Model 3: Adjusting for personal, environmental and health/illness related } \\
\text { factors }{ }^{\text {a }}\end{array}$} \\
\hline Characteristics & $\begin{array}{l}\text { Parameter } \\
\text { estimate }(\beta)\end{array}$ & SE & AOR & $95 \% \mathrm{CI}$ & $\begin{array}{c}\mathbf{P} \\
\text { Value }\end{array}$ \\
\hline \multicolumn{6}{|l|}{ TRD (Ref: No TRD) } \\
\hline Yes & 0.44 & 0.050 & 1.55 & {$[1.39,1.71]$} & $<0.001$ \\
\hline Time & -0.048 & 0.004 & - & - & $<0.001$ \\
\hline TRD*Time & -0.033 & 0.012 & - & - & 0.006 \\
\hline \multicolumn{6}{|c|}{ Age Group (Ref $: \geq 65$ years) } \\
\hline $18-34$ years & -0.18 & 0.179 & 0.86 & {$[0.72,1.02]$} & 0.102 \\
\hline $35-44$ years & 0.92 & 0.146 & 1.51 & {$[1.31,1.73]$} & $<0.001$ \\
\hline $45-54$ years & 1.49 & 0.123 & 1.87 & {$[1.66,2.10]$} & $<0.001$ \\
\hline $55-64$ years & 1.68 & 0.109 & 2.03 & {$[1.84,2.25]$} & $<0.001$ \\
\hline \multicolumn{6}{|l|}{ Sex (Ref: male) } \\
\hline Female & -0.72 & 0.078 & 0.74 & {$[0.69,0.80]$} & $<0.001$ \\
\hline \multicolumn{6}{|c|}{ Index Year (Ref: Years 2008-2010) } \\
\hline Years 2011-2013 & -0.32 & 0.087 & 0.86 & {$[0.79-0.94]$} & $<0.001$ \\
\hline Years 2014-2015 & -0.85 & 0.090 & 0.66 & {$[0.61-0.72]$} & $<0.001$ \\
\hline \multicolumn{6}{|c|}{ No. of CNPC $^{b}$ (Ref: 1 CNPC) } \\
\hline 2 & 1.30 & 0.108 & 1.85 & {$[1.65,2.07]$} & $<0.001$ \\
\hline$\geq 3$ & 3.25 & 0.112 & 4.20 & {$[3.75,4.69]$} & $<0.001$ \\
\hline \multicolumn{6}{|c|}{ Number of physical chronic conditions ${ }^{\mathrm{c}}($ Ref: 0$)$} \\
\hline 1 to 2 & 0.28 & 0.088 & 1.25 & {$[1.14,1.36]$} & $<0.001$ \\
\hline$\geq 3$ & 0.17 & 0.104 & 1.21 & {$[1.09,1.34]$} & $<0.001$ \\
\hline \multicolumn{6}{|c|}{ Anxiety disorders (Ref No) } \\
\hline Yes & 0.22 & 0.075 & 1.12 & {$[1.04,1.20]$} & 0.001 \\
\hline \multicolumn{6}{|c|}{ Sleep disorder (Ref: No) } \\
\hline Yes & 0.25 & 0.078 & 1.08 & {$[1.01,1.17]$} & 0.025 \\
\hline \multicolumn{6}{|c|}{ Polypharmacy (Ref: No) } \\
\hline Yes & 1.90 & 0.051 & 1.82 & {$[1.75,1.90]$} & $<0.001$ \\
\hline \multicolumn{6}{|l|}{ NSAIDs Use (Ref: No) } \\
\hline Yes & 0.36 & 0.039 & 1.12 & {$[1.08,1.16]$} & $<0.001$ \\
\hline
\end{tabular}




\section{Benzodiazepine use (Ref: no)}

Yes

$\begin{array}{lllll}1.00 & 0.044 & 1.44 & {[1.38,1.49]} & <0.001\end{array}$

Abbreviations: MDD: Major Depressive Disorder, TRD: Treatment- resistant depression,

CNPC: Chronic non-cancer pain conditions, NSAID: Non-steroidal anti-inflammatory drugs,

OR: Odds ratio, AOR: Adjusted odds ratio, CI: Confidence Interval

Note: Based on 21,599 adults with CNPC and newly diagnosed MDD receiving antidepressant medication treatment who were continuously enrolled during the observation period.

Long-term opiod therapy was defined as at least a $>90$ days of continuous supply of prescription opioids within a 180 -day period

${ }^{a}$ Only selected statistically variables are presented. The fully adjusted model adjusted for all personal factors (age, sex, insurance plan type, Medicare Advantage Enrollment),

environmental factors (region of residence, index year), and health and illness factors (number of physical chronic conditions, number of CNPC, presence of anxiety disorders, sleep disorders, obesity, and medication use (polypharmacy, NSAID, and benzodiazepine use). Polypharmacy, NSAIDs and benzodiazepines used as time varying variables and were measured at all six time points.

${ }^{b}$ Number of CNPC conditions were obtained as sum of common chronic painful conditions such as back/neck pain, headache/migraine, arthritis/joint pain, and neuropathic pain/other chronic pain conditions.

${ }^{c}$ Physical chronic conditions examined included conditions other than CNPC conditions such as asthma, coronary artery disease, cardiac arrhythmias, congestive heart failure, chronic kidney disease, chronic obstructive pulmonary disease, diabetes, hypertension, hyperlipidemia, osteoporosis, and stroke. 


\section{CHAPTER 5}

\section{Summary and Conclusion}

\subsection{Summary of Findings and Discussion}

Treatment-resistant depression, commonly defined as insufficient response to multiple antidepressant trials and augmentation strategies, ${ }^{17-20}$ remains highly prevalent and exacts a substantial clinical, economic, and humanistic burden among adults with MDD. ${ }^{20}$ Managing TRD in patients with CNPC and MDD is even more challenging because of the complex bidirectional relationship between depression and pain, which often exacerbates one another, and reduces benefits of depression and pain treatment. ${ }^{14,11,15}$ Existing literature on TRD has focused on all adults with MDD. Owing to the lack of research on TRD among adults with CNPC and MDD, we conducted this study to fill a critical knowledge gap and inform early interventions for reducing the risk of transitioning to TRD.

Given that MDD and CNPC are both very costly conditions, ${ }^{8,11,157}$ and the presence of TRD can further amplify this economic burden, it is important to estimate the incremental economic burden associated with TRD. Estimating excess healthcare cost associated with TRD can establish benchmarks for future cost of illness and cost-effectiveness studies, and can help in translating the burden of illness into dollar terms, the universal language for healthcare decision making. ${ }^{49}$ Many healthcare policy initiatives have shifted focus to the pursuit of triple aim of improving health outcomes, quality of care and reducing healthcare costs. ${ }^{48}$ This requires identification of not only high-cost but also high-risk and high-need patients. Despite recommendations against long-term opioid use among adults with CNPC and depression, LTOT continues to be prescribed to adults with CNPC and MDD in real-world. ${ }^{56,60,137,138}$ LTOT use 
among adults with TRD may make them a high-risk, high-need group as both LTOT and TRD are independently associated with high risk of substance-use disorders, including abuse and misuse of opioids, suicidal ideation and risk of hospitalizations. ${ }^{20,22,54,139}$ Therefore, examining the trajectory of LTOT among patients with CNPC and newly diagnosed MDD, and the association of TRD with LTOT can inform clinical practice, payers, and policy makers in their surveillance efforts of controlling opioid- related misuse and abuse in these patients.

Therefore, this dissertation pursued the following three related aims: 1) identifying leading predictors of TRD among adults with CNPC and newly diagnosed MDD; 2) estimating the direct economic burden associated with TRD and identifying factors that contribute to the excess cost burden of TRD; and 3) examining the trajectory of LTOT use in adults with CNPC and newly diagnosed MDD and how TRD can alter this trajectory. The current study, which focuses on predictors and outcomes associated with TRD among patients with CNPC and MDD is timely considering recent advances in the development of treatment options for TRD. After a period in which it seemed that the innovation of antidepressants with new mechanisms of action was going dry, the past decade has witnessed a renewed interest starting with the discovery of antidepressant properties of ketamine and other newer investigational antidepressants. ${ }^{158}$ The current study further highlights that there remains an unmet need for adequate treatment options to manage chronic pain and TRD, and suggests that patient with TRD and CNPC have a significant illness burden.

\section{Leading Predictors of TRD among adults with CNPC and newly diagnosed MDD}

We found that one in every nine adults with CNPC and newly diagnosed MDD transitioned to TRD within one year of MDD diagnosis. As demonstrated in this study, a smaller set of more easily assessed factors during the time of MDD diagnosis can be used to gauge the 
risk of transitioning to TRD. The leading predictors identified using robust machine learning approaches included mental health specialist visits, use of psychotherapy after MDD diagnosis, polypharmacy, age, presence of comorbid anxiety disorders, use of benzodiazepines, inpatient status, index antidepressant class, and number of CNPC conditions. These factors may serve as targets of further investigation or clinical intervention to improve treatment outcomes in this population

\section{Impact of TRD on direct economic burden}

Adults with CNPC who transitioned from MDD to TRD had significantly higher direct all-cause and MDD-related costs and healthcare resource utilization. Use of inpatient services and prescription drug costs accounted for a significant portion of the total all-cause costs. It is intuitive that adults with TRD will have higher prescription drug costs because of failure to respond to multiple trials of antidepressants, higher illness burden due to the presence of other chronic conditions, and higher use of polypharmacy. Our findings indicate that interventions focused on reducing the risk of transitioning to TRD may have the potential to prevent inpatient hospitalizations and can lead to cost savings for payers.

\section{Factors contributing to the excess cost burden of TRD}

Our findings indicated that $46 \%$ of the excess total costs among adults with TRD were explained by differences in patient level characteristics such as polypharmacy, number of CNPC, anxiety disorders, sleep disorders, and substance use disorders between the TRD and no TRD groups. Therefore, potential cost savings associated with managing TRD may stem from reducing the risk of polypharmacy, better co-management of chronic pain and co-occurring substance use, anxiety, and sleep disorders. 


\section{Trajectory of LTOT among adults with CNPC and MDD and association of TRD with}

\section{LTOT}

Patients with TRD were significantly more likely to receive LTOT as compared to adults with CNPC and MDD who did not meet the criteria for TRD at all time points during the 3 year study period. The reasons for the higher use of LTOT among adults with TRD are not clear. Future research needs to explore the reasons as to why TRD patients were more likely to receive LTOT despite treatment guidelines recommending against the use of LTOT in subgroup of adults with CNPC and MDD. Additionally, these findings further reinforce the need for specific clinical guidelines for pain management with opioids among adults with CNPC and MDD who meet the criteria for TRD and suggest that clinicians need to closely monitor the risks of using LTOT in this subgroup.

On the other hand, we observed that LTOT use declined at a higher rate among adults with TRD as compared to those without TRD in our study sample. These findings are encouraging and suggest that clinicians may decide to taper and discontinue the use of LTOT among adults with TRD perhaps due to lack of pain relief, higher risk of opioid -related side effects and adverse events such as risk of addiction, opioid dependence and misuse.

\subsection{Implications and Suggestions for Future Research}

Our study findings suggest that an individual's transition from MDD to TRD among patients with CNPC can be predicted by information readily available in clinical settings such as presence of comorbid anxiety disorders, history of inpatient hospitalization, younger age, presence of higher number of CNPC, and indications of higher severity of MDD such as use of psychotherapy, and mental health specialist visits. The study findings provide some actionable evidence that can be utilized in real-world treatment decisions. For example, when a patient with 
CNPC and newly diagnosed MDD first visits a healthcare provider, the presence of these characteristics may alert him/her to the possibility that the patient could develop TRD. Monitoring patients with these characteristics more closely, using targeted individualized treatment approaches, and using approaches such as augmentation strategies earlier in the treatment process may reduce the risk of TRD. There is a need to shift the focus of care from disease-specific to patient-centered care. The current study also highlights a huge unmet need for management of TRD among patients with CNPC and MDD. As the focus on developing newer treatment approaches for TRD is increasing in the past few years, ${ }^{158}$ there is a need for studies examining the efficacy and effectiveness of these medications on not only alleviating depression symptoms but also pain symptoms. As MDD and chronic pain have overlapping neurobiological pathways, they often respond to similar treatments and therefore, effectively managing one symptom may also affect the other symptom. ${ }^{14,15}$ Another important research implication is to test the validity of the leading predictors obtained from the current study using other datasets. Future studies also need to include a more comprehensive set of clinical variables that could not be examined in the current study, including, levels of inflammatory cytokines, pain scores, and psychological stress to identify predictors of TRD among patients with CNPC.

The current study is also the first study of its kind to estimate the direct economic burden associated with TRD among adults with CNPC and MDD. Therefore, quantification of the excess cost burden of TRD helps in translating the burden of illness into dollar terms which can be used as input parameters for future cost-effectiveness studies. Our findings can also inform payers and other stake-holders. As there is an emerging trend to focus on "value-based" care, which emphasize on quality care at lower costs, alternative payment models such as bundled payments, accountable care organizations, and medical homes are now increasingly used. As 
bundled payment models require risk adjustment to account for differences in beneficiary-case mix ${ }^{159}$ our findings suggest that an up to date estimate of economic burden for adults with MDD should also include excess costs due to TRD. As TRD is associated with a significantly high economic burden among patients with CNPC and MDD, our findings suggest that resource allocation for MDD-related expenditures should be higher for patients with TRD.

The current study also highlights the patient level differences between adults with and without TRD that contribute to the excess cost burden of TRD using post-decomposition techniques. Our results suggest that the excess cost burden associated with TRD could be reduced by adequately managing polypharmacy, chronic pain, comorbid sleep, anxiety and substance-use disorders. Our findings suggest that interventions that have shown to reduce the risk of polypharmacy such as systematic review of high risk medications, medication therapy management, medication reconciliation, and using a multidisciplinary care approach ${ }^{114}$ have the potential to off-set the excess costs due to polypharmacy. Furthermore, existing research shows that the complex need of patients with multiple chronic conditions can be better managed through healthcare delivery models using multidisciplinary and collaborative care approaches. ${ }^{122,123}{ }^{124}$ Therefore, patient-centered care by integrating mental and physical health in primary care settings could improve treatment outcomes and potentially reduce costs associated with TRD.

Our findings also provide clinical and policy implications regarding use of LTOT among patients with CNPC and newly diagnosed MDD. Consistently higher LTOT use among adults with TRD across all time periods suggests the need for providers to closely monitor patients for the risk of opioid related side-effects and adverse events such as opioid misuse, abuse and dependence. Providers may need to weigh the risks versus benefits of using LTOT in this patient 
population. Real-world findings on patterns of LTOT use among patients with CNPC and MDD can further aid clinicians and policy makers in their surveillance efforts of combating the current opioid crisis. Despite the increased rate of LTOT use among patients with CNPC and MDD, data are lacking on the efficacy and safety of LTOT for CNPC, and particularly for patients with MDD or substance abuse who are generally excluded from randomized trials. ${ }^{33}$ Additionally, lack of data on efficacy and effectiveness of LTOT among adults with CNPC and TRD warrants well-designed pragmatic trials to provide evidence on the effectiveness of LTOT use. There is also a need for evidence-based clinical guidelines for use of LTOT in vulnerable groups such as adults with CNPC and TRD. Our study findings also highlight the need for future studies to ascertain clinical and patient-related reasons for high rates of LTOT use among patients with TRD. Given, that a study by Scherrer et al. has reported that LTOT is a risk factor for TRD, ${ }^{30}$ and our study observed association of TRD with LTOT, future real-world studies are needed to explore the bidirectional relationships between LTOT and TRD.

\subsection{Strengths and Limitations}

The study has several strengths, including availability of a large cohort of patients with CNPC and MDD treated for depression in a real-world setting, and longitudinal study design to track individuals over time, thereby enabling assessment of temporal relationships. Our study used a comprehensive algorithm that encompasses the four main strategies to overcome lack of response, including optimization, switching, combination, and augmentation ${ }^{62,106}$ to identify adults with TRD. Additionally, the current study ensured robustness of study findings by conducting sensitivity analyses, adjusting for observed selection bias through IPTWs, and using advanced statistical techniques such as counterfactual-recycled predictions, two-part GLM model, generalized estimating equations, and machine learning using random forest. The use of 
standardized prices for medical services, which adjusts for changes in payments based on local factors, such as labor costs allowed for more accurate comparisons of spending on healthcare services. ${ }^{160}$

Our study findings should be interpreted in the context of some limitations. First, the study suffers from inherent limitations associated with claims data analysis, including coding inaccuracies, absence of information on the severity of MDD, socioeconomic status, race/ethnicity, physical activity, and social support. Second, TRD was defined using pharmacy claims and ECT use and excludes other clinical considerations such as type of symptoms, responsiveness to medical treatments, and tolerance to antidepressants which may under-estimate or over-estimate the prevalence of TRD. Third, we may not have truly captured the actual use of medication, as we could observe only presence of prescription claims. It is also plausible that by limiting patients to only MDD diagnosis, we may have underestimated the prevalence of MDD because MDD may at times could also be recorded using a general depression code. Finally, although our study was not limited to including only working-aged adults, our results may not be generalizable to other privately and publicly insured adults.

\subsection{Conclusion}

Overall, we identified that mental health specialist visits, polypharmacy $(\geq 5$ medications), psychotherapy use, presence of comorbid anxiety disorders, and age were the five leading predictors of TRD. TRD is associated with a high direct economic burden in terms of allcause and MDD-related costs and utilization among adults with CNPC and newly diagnosed MDD. Additionally, we observed that LTOT rates were consistently higher among TRD patients as compared to those without TRD across all time points; although the decline in LTOT was greater in patients with TRD as compared to those without TRD. Although this study fills a 
critical knowledge gap, future research needs to confirm our study findings by including MDD severity, pain scores, level of inflammatory biomarkers, social support, and other unobserved factors such as income level, education, race and ethnicity that may affect the relationship between TRD and economic and clinical outcomes. Future studies also need to incorporate these factors when examining the risk factors of TRD among adults with CNPC and MDD. Our findings suggest that use of multidisciplinary, collaborative, and targeted behavioral approach is needed to not only better manage patients with CNPC and TRD who use LTOT but also to better manage multimorbidity, polypharmacy, and to reduce the risk of inpatient hospitalizations. These approaches can also have the potential to reduce the excess costs associated with TRD. 


\section{References}

1. Ferrari AJ, Charlson FJ, Norman RE, et al. Burden of depressive disorders by country, sex, age, and year: findings from the global burden of disease study 2010. PLoS medicine. 2013;10(11):e1001547.

2. Kessler RC, Berglund P, Demler O, et al. The epidemiology of major depressive disorder: results from the National Comorbidity Survey Replication (NCS-R). Jama. 2003;289(23):3095-3105.

3. Kessler RC, Petukhova M, Sampson NA, Zaslavsky AM, Wittchen HU. Twelve-month and lifetime prevalence and lifetime morbid risk of anxiety and mood disorders in the United States. International journal of methods in psychiatric research. Sep 2012;21(3):169-184.

4. Watson L, Baird J, Hösel V, Peveler R. The effect of concurrent pain on the management of patients with depression: an analysis of NHS healthcare resource utilisation using the GPRD database. International Journal of clinical practice. 2009;63(5):698-706.

5. Grattan A, Sullivan MD, Saunders KW, Campbell CI, Von Korff MR. Depression and prescription opioid misuse among chronic opioid therapy recipients with no history of substance abuse. The Annals of Family Medicine. 2012;10(4):304-311.

6. Hasin DS, Goodwin RD, Stinson FS, Grant BF. Epidemiology of major depressive disorder: results from the National Epidemiologic Survey on Alcoholism and Related Conditions. Arch Gen Psychiatry. Oct 2005;62(10):1097-1106.

7. Bair MJ, Robinson RL, Katon W, Kroenke K. Depression and pain comorbidity: a literature review. Archives of internal medicine. 2003;163(20):2433-2445.

8. Greenberg PE, Fournier A-A, Sisitsky T, Pike CT, Kessler RC. The economic burden of adults with major depressive disorder in the United States (2005 and 2010). The Journal of clinical psychiatry. 2015;76(2):155-162.

9. Cuijpers P, Beekman AT, Reynolds CF. Preventing depression: a global priority. Jama. 2012;307(10):1033-1034.

10. Katon WJ. Epidemiology and treatment of depression in patients with chronic medical illness. Dialogues in clinical neuroscience. 2011;13(1):7.

11. Bair MJ, Robinson RL, Eckert GJ, Stang PE, Croghan TW, Kroenke K. Impact of pain on depression treatment response in primary care. Psychosomatic medicine. 2004;66(1):17-22.

12. Kroenke K, Wu J, Bair MJ, Krebs EE, Damush TM, Tu W. Reciprocal relationship between pain and depression: a 12-month longitudinal analysis in primary care. The Journal of Pain. 2011;12(9):964-973.

13. Kapfhammer H-P. Somatic symptoms in depression. Dialogues in Clinical Neuroscience. 2006;8(2):227-239.

14. Lindsay PG, Wyckoff $M$. The depression-pain syndrome and its response to antidepressants. Psychosomatics. 1981;22(7):571-573.

15. Gallagher R, Verma S. Managing pain and comorbid depression: A public health challenge. Paper presented at: Seminars in clinical neuropsychiatry1999.

16. Sheng J, Liu S, Wang Y, Cui R, Zhang X. The Link between Depression and Chronic Pain: Neural Mechanisms in the Brain. Neural plasticity. 2017;2017:9724371-9724371.

17. Nemeroff CB. Prevalence and management of treatment-resistant depression. Journal of Clinical Psychiatry. 2007;68(8):17. 
18. Nierenberg AA, DeCecco LM. Definitions of antidepressant treatment response, remission, nonresponse, partial response, and other relevant outcomes: a focus on treatment-resistant depression. The Journal of clinical psychiatry. 2001;62:5-9.

19. Fava M, Rush AJ, Wisniewski SR, et al. A comparison of mirtazapine and nortriptyline following two consecutive failed medication treatments for depressed outpatients: a STAR* D report. American Journal of Psychiatry. 2006;163(7):1161-1172.

20. Mrazek DA, Hornberger JC, Altar CA, Degtiar I. A review of the clinical, economic, and societal burden of treatment-resistant depression: 1996-2013. Psychiatric services (Washington, D.C.). Aug 01 2014;65(8):977-987.

21. Rush AJ, Trivedi MH, Wisniewski SR, et al. Acute and longer-term outcomes in depressed outpatients requiring one or several treatment steps: a STAR* D report. American Journal of Psychiatry. 2006;163(11):1905-1917.

22. Kubitz N, Mehra M, Potluri RC, Garg N, Cossrow N. Characterization of treatment resistant depression episodes in a cohort of patients from a US commercial claims database. PloS one. 2013;8(10):e76882.

23. Gibson TB, Jing Y, Smith CG, et al. Cost burden of treatment resistance in patients with depression. The American journal of managed care. 2010;16(5):370-377.

24. Rizvi SJ, Grima E, Tan M, et al. Treatment-Resistant Depression in Primary Care Across Canada. Canadian Journal of Psychiatry. Revue Canadienne de Psychiatrie. 2014;59(7):349-357.

25. Crown WH, Finkelstein S, Berndt ER, et al. The impact of treatment-resistant depression on health care utilization and costs. The Journal of clinical psychiatry. 2002;63(11):963971.

26. Kautzky A, Dold M, Bartova L, et al. Clinical factors predicting treatment resistant depression: affirmative results from the European multicenter study. Acta Psychiatrica Scandinavica. 2018.

27. Kautzky A, Baldinger-Melich P, Kranz GS, et al. A New Prediction Model for Evaluating Treatment-Resistant Depression. The Journal of clinical psychiatry. 2017;78(2):215-222.

28. Kautzky A, Dold M, Bartova L, et al. Refining Prediction in Treatment-Resistant Depression: Results of Machine Learning Analyses in the TRD III Sample. The Journal of clinical psychiatry. 2018;79(1).

29. Cepeda MS, Reps J, Ryan P. Finding factors that predict treatment-resistant depression: Results of a cohort study. Depression and anxiety. 2018.

30. Scherrer JF, Salas J, Sullivan MD, et al. The Influence of Prescription Opioid Use Duration and Dose on Development of Treatment Resistant Depression. Preventive medicine. 08/03 2016;91:110-116.

31. Köhler O, Benros ME, Nordentoft M, et al. Effect of anti-inflammatory treatment on depression, depressive symptoms, and adverse effects: a systematic review and metaanalysis of randomized clinical trials. JAMA psychiatry. 2014;71(12):1381-1391.

32. Gallagher PJ, Castro V, Fava M, et al. Antidepressant response in patients with major depression exposed to NSAIDs: a pharmacovigilance study. American Journal of Psychiatry. 2012;169(10):1065-1072.

33. Kalso E, Allan L, Dellemijn PL, et al. Recommendations for using opioids in chronic non-cancer pain. European Journal of Pain. 2003;7(5):381-386. 
34. Avouac J, Gossec L, Dougados M. Efficacy and safety of opioids for osteoarthritis: a meta-analysis of randomized controlled trials. Osteoarthritis and Cartilage. 2007;15(8):957-965.

35. Songer D. Psychotherapeutic approaches in the treatment of pain. Psychiatry (Edgmont). 2005;2(5):19.

36. Qato D, Ozenberger K, Olfson M. Prevalence of prescription medications with depression as a potential adverse effect among adults in the united states. JAMA. 2018;319(22):2289-2298.

37. Bleakley S. Antidepressant drug interactions: evidence and clinical significance. Progress in Neurology and Psychiatry. 2016;20(3):21-27.

38. Regierer B, Zazzu V, Sudbrak R, Kühn A, Lehrach H, Consortium I. Future of medicine: models in predictive diagnostics and personalized medicine. Molecular Diagnostics: Springer; 2013:15-33.

39. Ng K, Ghoting A, Steinhubl SR, Stewart WF, Malin B, Sun J. PARAMO: a PARAllel predictive MOdeling platform for healthcare analytic research using electronic health records. Journal of biomedical informatics. 2014;48:160-170.

40. Gillum LA, Gouveia C, Dorsey ER, et al. NIH disease funding levels and burden of disease. PloS one. 2011;6(2):e16837.

41. Corey-Lisle PK, Birnbaum HG, Greenberg PE, Marynchenko MB, Claxton AJ. Identification of a claims data" signature" and economic consequences for treatmentresistant depression. The Journal of clinical psychiatry. 2002;63(8):717-726.

42. Greenberg P, Corey-Lisle PK, Marynchenko M, Claxton A. Economic implications of treatment-resistant depression among employees. Pharmacoeconomics. 2004;22(6):363373.

43. Lepine BA, Moreno RA, Campos RN, Couttolenc BF. Treatment-resistant depression increases health costs and resource utilization. Revista Brasileira de Psiquiatria. 2012;34(4):379-388.

44. Ivanova JI, Birnbaum HG, Kidolezi Y, Subramanian G, Khan SA, Stensland MD. Direct and indirect costs of employees with treatment-resistant and non-treatment-resistant major depressive disorder. Current medical research and opinion. Oct 2010;26(10):24752484.

45. Olchanski N, Myers MM, Halseth M, et al. The economic burden of treatment-resistant depression. Clinical therapeutics. 2013;35(4):512-522.

46. Amos TB, Tandon N, Lefebvre P, et al. Direct and Indirect Cost Burden and Change of Employment Status in Treatment-Resistant Depression: A Matched-Cohort Study Using a US Commercial Claims Database. The Journal of clinical psychiatry. 2018;79(2).

47. Bao Y, Sturm R, Croghan TW. A national study of the effect of chronic pain on the use of health care by depressed persons. Psychiatric Services. 2003;54(5):693-697.

48. Berwick DM, Nolan TW, Whittington J. The triple aim: care, health, and cost. Health affairs. 2008;27(3):759-769.

49. Rice DP. Cost of illness studies: what is good about them? Injury Prevention. 2000;6(3):177-179.

50. Balestri M, Calati R, Souery D, et al. Socio-demographic and clinical predictors of treatment resistant depression: a prospective European multicenter study. Journal of affective disorders. 2016;189:224-232. 
51. Bair MJ, Wu J, Damush TM, Sutherland JM, Kroenke K. Association of depression and anxiety alone and in combination with chronic musculoskeletal pain in primary care patients. Psychosomatic medicine. 2008;70(8):890.

52. Han C, Pae C-U. Pain and depression: a neurobiological perspective of their relationship. Psychiatry investigation. 2015;12(1):1-8.

53. Von Korff M, Deyo RA. Potent opioids for chronic musculoskeletal pain: flying blind? : LWW; 2004.

54. Chou R, Deyo R, Devine B, et al. The Effectiveness and Risks of Long-Term Opioid Treatment of Chronic Pain. Evidence report/technology assessment. Sep 2014(218):1219.

55. Sullivan MD. Depression effects on long-term prescription opioid use, abuse, and addiction. The Clinical journal of pain. 2018;34(9):878-884.

56. Braden JB, Sullivan MD, Ray GT, et al. Trends in long-term opioid therapy for noncancer pain among persons with a history of depression. General hospital psychiatry. 2009;31(6):564-570.

57. Sullivan MD. What are we treating with opioid and sedative-hypnotic combination therapy? Pharmacoepidemiol Drug Saf. Aug 2015;24(8):893-895.

58. Smith H, Passik S. Pain and chemical dependency. Oxford University Press; 2008.

59. Sullivan MD, Edlund MJ, Fan M-Y, DeVries A, Braden JB, Martin BC. Risks for possible and probable opioid misuse among recipients of chronic opioid therapy in commercial and medicaid insurance plans: The TROUP Study. Pain. 2010;150(2):332339.

60. Sullivan MD, Edlund MJ, Zhang L, Unützer J, Wells KB. Association between mental health disorders, problem drug use, and regular prescription opioid use. Archives of internal medicine. 2006;166(19):2087-2093.

61. Petersen T, Papakostas GI, Posternak MA, et al. Empirical testing of two models for staging antidepressant treatment resistance. Journal of clinical psychopharmacology. 2005;25(4):336-341.

62. Al-Harbi KS. Treatment-resistant depression: therapeutic trends, challenges, and future directions. Patient preference and adherence. 2012;6:369-388.

63. Wilkinson RG, Marmot M. Social determinants of health: the solid facts. World Health Organization; 2003.

64. Andersen RM. Revisiting the behavioral model and access to medical care: does it matter? Journal of health and social behavior. 1995:1-10.

65. Dodd M, Janson S, Facione N, et al. Advancing the science of symptom management. Journal of advanced nursing. 2001;33(5):668-676.

66. Linder L. Analysis of the UCSF Symptom Management Theory: implications for pediatric oncology nursing. Journal of pediatric oncology nursing : official journal of the Association of Pediatric Oncology Nurses. Nov-Dec 2010;27(6):316-324.

67. Optum. Optum Clinformatics Data Mart[cited 2018 June 7].Available from: https://www.optum.com/content/dam/optum/resources/productSheets/Clinformatics_for_ Data_Mart.pdf. 2018.

68. World Health Organization Depression: A Global public health concern. Department of Mental Health and Substance Abuse. 2012.

69. Friedrich M. Depression is the leading cause of disability around the world. Jama. 2017;317(15):1517-1517. 
70. Khan A, Brown WA. Antidepressants versus placebo in major depression: an overview. World Psychiatry. 2015;14(3):294-300.

71. Kirsch I, Huedo-Medina TB, Pigott HE, Johnson BT. Do outcomes of clinical trials resemble those "real world" patients? A reanalysis of the STAR* D antidepressant data set. Psychology of Consciousness: Theory, Research, and Practice. 2018.

72. Conway CR, George MS, Sackeim HA. Toward an evidence-based, operational definition of treatment-resistant depression: when enough is enough. JAMA psychiatry. 2017;74(1):9-10.

73. Nie Z, Vairavan S, Narayan VA, Ye J, Li QS. Predictive modeling of treatment resistant depression using data from STAR* D and an independent clinical study. PloS one. 2018;13(6):e0197268.

74. De Carlo V, Calati R, Serretti A. Socio-demographic and clinical predictors of nonresponse/non-remission in treatment resistant depressed patients: a systematic review. Psychiatry research. 2016;240:421-430.

75. Giummarra MJ, Gibson SJ, Allen AR, Pichler AS, Arnold CA. Polypharmacy and chronic pain: harm exposure is not all about the opioids. Pain Medicine. 2015;16(3):472479.

76. Taylor Jr R, V Pergolizzi Jr J, Puenpatom RA, Summers KH. Economic implications of potential drug-drug interactions in chronic pain patients. Expert review of pharmacoeconomics \& outcomes research. 2013;13(6):725-734.

77. PHYTEL. Predictive Modeling and Population Health Management. Whitepaper 2013; https://app.compendium.com/uploads/user/863cc3c6-3316-459a-a7473323bd3b6428/4c5909e8-1708-4751-873e4129cb2ed878/File/86f9d9a5e2f21fb11a5ad8ef2239a76e/1393861691669.pdf.

78. Schultz J, Joish V. Costs associated with changes in antidepressant treatment in a managed care population with major depressive disorder. Psychiatric Services. 2009;60(12):1604-1611.

79. Johnson ES, Bartman BA, Briesacher BA, et al. The incident user design in comparative effectiveness research. Pharmacoepidemiology and drug safety. 2013;22(1):1-6.

80. Scherrer JF, Svrakic DM, Freedland KE, et al. Prescription opioid analgesics increase the risk of depression. Journal of general internal medicine. 2014;29(3):491-499.

81. Sullivan MD, Edlund MJ, Fan M-Y, DeVries A, Braden JB, Martin BC. Trends in use of opioids for non-cancer pain conditions 2000-2005 in commercial and Medicaid insurance plans: the TROUP study. Pain. 2008;138(2):440-449.

82. Shah A, Hayes CJ, Martin BC. Factors influencing long-term opioid use among opioid naive patients: An examination of initial prescription characteristics and pain etiologies. The Journal of Pain. 2017;18(11):1374-1383.

83. Marcus RN, McQuade RD, Carson WH, et al. The efficacy and safety of aripiprazole as adjunctive therapy in major depressive disorder: a second multicenter, randomized, double-blind, placebo-controlled study. Journal of clinical psychopharmacology. 2008;28(2):156-165.

84. Shmueli G. To explain or to predict? Statistical science. 2010;25(3):289-310.

85. Breiman L. Statistical modeling: The two cultures (with comments and a rejoinder by the author). Statistical science. 2001;16(3):199-231.

86. Liaw A, Wiener M. Classification and regression by randomForest. $R$ news. 2002;2(3):18-22. 
87. Rao SJ. Regression modeling strategies: with applications to linear models, logistic regression, and survival analysis. Taylor \& Francis; 2003.

88. Masnoon N, Shakib S, Kalisch-Ellett L, Caughey GE. What is polypharmacy? A systematic review of definitions. BMC geriatrics. 2017;17(1):230.

89. Saeys Y, Inza I, Larrañaga P. A review of feature selection techniques in bioinformatics. bioinformatics. 2007;23(19):2507-2517.

90. Murphy JA, Sarris J, Byrne GJ. A review of the conceptualisation and risk factors associated with treatment-resistant depression. Depression research and treatment. 2017;2017.

91. Bennabi D, Aouizerate B, El-Hage W, et al. Risk factors for treatment resistance in unipolar depression: a systematic review. Journal of affective disorders. 2015;171:137141.

92. Tsang A, Von Korff M, Lee S, et al. Common chronic pain conditions in developed and developing countries: gender and age differences and comorbidity with depressionanxiety disorders. The journal of pain. 2008;9(10):883-891.

93. American Psychiatric Association. Practice guideline for the treatment of patients with major depressive disorder (3rd). http://psychiatryonline. org/guidelines. aspx. 2009.

94. Unützer J, Park M. Strategies to improve the management of depression in primary care. Primary Care: Clinics in Office Practice. 2012;39(2):415-431.

95. Liu X, Ye W, Watson P, Tepper P. Use of benzodiazepines, hypnotics, and anxiolytics in major depressive disorder: association with chronic pain diseases. The Journal of nervous and mental disease. 2010;198(8):544-550.

96. Cunningham JL, Craner JR, Evans MM, Hooten WM. Benzodiazepine use in patients with chronic pain in an interdisciplinary pain rehabilitation program. Journal of Pain Research. 2017;10:311.

97. Morgan SG, Weymann D, Pratt B, et al. Sex differences in the risk of receiving potentially inappropriate prescriptions among older adults. Age and ageing. Jul 2016;45(4):535-542.

98. Nielsen S, Lintzeris N, Bruno R, et al. Benzodiazepine use among chronic pain patients prescribed opioids: associations with pain, physical and mental health, and health service utilization. Pain medicine (Malden, Mass.). Feb 2015;16(2):356-366.

99. Markota M, Rummans TA, Bostwick JM, Lapid MI. Benzodiazepine use in older adults: dangers, management, and alternative therapies. Paper presented at: Mayo Clinic Proceedings2016.

100. Howland RH, Wilson MG, Kornstein SG, et al. Factors predicting reduced antidepressant response: experience with the SNRI duloxetine in patients with major depression. Annals of clinical psychiatry : official journal of the American Academy of Clinical Psychiatrists. Oct-Dec 2008;20(4):209-218.

101. Du Vaure CB, Dechartres A, Battin C, Ravaud P, Boutron I. Exclusion of patients with concomitant chronic conditions in ongoing randomised controlled trials targeting 10 common chronic conditions and registered at ClinicalTrials. gov: a systematic review of registration details. BMJ open. 2016;6(9):e012265.

102. Welch CA, Czerwinski D, Ghimire B, Bertsimas D. Depression and costs of health care. Psychosomatics. Jul-Aug 2009;50(4):392-401. 
103. Rayner L, Hotopf M, Petkova H, Matcham F, Simpson A, McCracken LM. Depression in patients with chronic pain attending a specialised pain treatment centre: prevalence and impact on health care costs. Pain. 2016;157(7):1472.

104. Arnow BA, Hunkeler EM, Blasey CM, et al. Comorbid depression, chronic pain, and disability in primary care. Psychosomatic medicine. 2006;68(2):262-268.

105. Thase ME, Greenhouse JB, Frank E, et al. Treatment of major depression with psychotherapy or psychotherapy-pharmacotherapy combinations. Archives of General Psychiatry. 1997;54(11):1009-1015.

106. Johnston KM, Powell LC, Anderson IM, Szabo S, Cline S. The burden of treatmentresistant depression: A systematic review of the economic and quality of life literature. Journal of affective disorders. 2019;242:195-210.

107. Deb P, Norton EC. Modeling Health Care Expenditures and Use. Annual review of public health. Apr 1 2018;39:489-505.

108. Basu A, Rathouz PJ. Estimating marginal and incremental effects on health outcomes using flexible link and variance function models. Biostatistics. Jan 2005;6(1):93-109.

109. Oaxaca R. Male-female wage differentials in urban labor markets. International economic review. 1973:693-709.

110. Blinder AS. Wage discrimination: reduced form and structural estimates. Journal of Human resources. 1973:436-455.

111. Mitra S, Findley PA, Sambamoorthi U. Health Care Expenditures of Living With a Disability: Total Expenditures, Out-of-Pocket Expenses, and Burden, 1996 to 2004. Archives of Physical Medicine and Rehabilitation. 2009;90(9):1532-1540.

112. Austin PC. An Introduction to Propensity Score Methods for Reducing the Effects of Confounding in Observational Studies. Multivariate behavioral research. 2011;46(3):399-424.

113. Vergunst FK, Fekadu A, Wooderson SC, et al. Longitudinal course of symptom severity and fluctuation in patients with treatment-resistant unipolar and bipolar depression. Psychiatry research. 2013;207(3):143-149.

114. Johansson $\mathrm{T}$, Abuzahra ME, Keller S, et al. Impact of strategies to reduce polypharmacy on clinically relevant endpoints: a systematic review and meta-analysis. British journal of clinical pharmacology. 2016;82(2):532-548.

115. Kojima G, Bell C, Tamura B, et al. Reducing cost by reducing polypharmacy: the polypharmacy outcomes project. Journal of the American Medical Directors Association. 2012;13(9):818.e811-818.e818.818E815.

116. Bergfeld IO, Mantione M, Figee M, Schuurman PR, Lok A, Denys D. Treatmentresistant depression and suicidality. Journal of affective disorders. Aug 1 2018;235:362367.

117. Pfeiffer PN, Kim HM, Ganoczy D, Zivin K, Valenstein M. Treatment-resistant depression and risk of suicide. Suicide \& life-threatening behavior. Aug 2013;43(4):356365.

118. Davydow DS, Fenger-Grøn M, Ribe AR, et al. Depression and risk of hospitalisations and rehospitalisations for ambulatory care-sensitive conditions in Denmark: a populationbased cohort study. BMJ open. 2015;5(12):e009878.

119. Citrome L, Jain R, Tung A, Landsman-Blumberg PB, Kramer K, Ali S. Prevalence, treatment patterns, and stay characteristics associated with hospitalizations for major depressive disorder. Journal of affective disorders. Feb 14 2019;249:378-384. 
120. Sambamoorthi U, Tan $X$, Deb A. Multiple chronic conditions and healthcare costs among adults. Expert review of pharmacoeconomics \& outcomes research. 2015;15(5):823-832.

121. Arnow BA, Blasey CM, Lee J, et al. Relationships among depression, chronic pain, chronic disabling pain, and medical costs. Psychiatric services (Washington, D.C.). Mar 2009;60(3):344-350.

122. Lin EH, Katon W, Von Korff M, et al. Effect of improving depression care on pain and functional outcomes among older adults with arthritis: a randomized controlled trial. Jama. 2003;290(18):2428-2429.

123. Pietruszewski P. A new direction in depression treatment in Minnesota: DIAMOND program. Institute for Clinical Systems Improvement, Bloomington, Minnesota. Psychiatr Serv. 2010;61(10):1042-1044.

124. Katon WJ, Lin EH, Von Korff M, et al. Collaborative care for patients with depression and chronic illnesses. New England Journal of Medicine. 2010;363(27):2611-2620.

125. Wong J, Motulsky A, Abrahamowicz M, Eguale T, Buckeridge DL, Tamblyn R. Offlabel indications for antidepressants in primary care: descriptive study of prescriptions from an indication based electronic prescribing system. BMJ. 2017;356:j603.

126. Von Korff M, Saunders K, Thomas Ray G, et al. De facto long-term opioid therapy for noncancer pain. Clin J Pain. Jul-Aug 2008;24(6):521-527.

127. Vanderlip ER, Sullivan MD, Edlund MJ, et al. National study of discontinuation of longterm opioid therapy among veterans. Pain®. 2014;155(12):2673-2679.

128. Edlund MJ, Martin BC, Russo JE, DeVries A, Braden JB, Sullivan MD. The role of opioid prescription in incident opioid abuse and dependence among individuals with chronic noncancer pain: the role of opioid prescription. The Clinical journal of pain. 2014;30(7):557-564.

129. AHRQ. The Effectiveness and Risks of Long-Term Opioid Treatment of Chronic Pain. 2014; http://effectivehealthcare.ahrq.gov/index.cfm/search-for-guides-reviews-andreports/?productid=1988\&pageaction=displayproduct, 2016.

130. Dowell D, Haegerich TM, Chou R. CDC Guideline for Prescribing Opioids for Chronic Pain-United States, 2016. JAMA. Mar 152016.

131. Breivik H. Opioids in chronic non-cancer pain, indications and controversies. European Journal of pain. 2005;9(2):127-130.

132. Portenoy RK. Appropriate use of opioids for persistent non-cancer pain. Lancet (London, England). Aug 28-Sep 3 2004;364(9436):739-740.

133. Busse JW, Wang L, Kamaleldin M, et al. Opioids for Chronic Noncancer Pain: A Systematic Review and Meta-analysisOpioids for Chronic Noncancer PainOpioids for Chronic Noncancer Pain. JAMA. 2018;320(23):2448-2460.

134. Noble M, Treadwell JR, Tregear SJ, et al. Long-term opioid management for chronic noncancer pain. The Cochrane database of systematic reviews. Jan 20 2010(1):Cd006605.

135. Furlan AD, Sandoval JA, Mailis-Gagnon A, Tunks E. Opioids for chronic noncancer pain: a meta-analysis of effectiveness and side effects. CMAJ : Canadian Medical Association journal = journal de l'Association medicale canadienne. May 23 2006;174(11):1589-1594.

136. Wasan AD, Michna E, Edwards RR, et al. Psychiatric Comorbidity Is Associated Prospectively with Diminished Opioid Analgesia and Increased Opioid Misuse in Patients with Chronic Low Back Pain. Anesthesiology. Oct 2015;123(4):861-872. 
137. Scherrer JF, Salas J, Copeland LA, et al. Prescription opioid duration, dose, and increased risk of depression in 3 large patient populations. The Annals of Family Medicine. 2016;14(1):54-62.

138. Davis MA, Lin LA, Liu H, Sites BD. Prescription opioid use among adults with mental health disorders in the United States. The Journal of the American Board of Family Medicine. 2017;30(4):407-417.

139. Kornstein SG, Schneider RK. Clinical features of treatment-resistant depression. Journal of Clinical Psychiatry. 2001;62:18-25.

140. Edlund MJ, Martin BC, Devries A, Fan M-Y, Braden JB, Sullivan MD. Trends in use of opioids for chronic non-cancer pain among individuals with mental health and substance use disorders: the TROUP study. The Clinical journal of pain. 2010;26(1):1.

141. Fava M. Diagnosis and definition of treatment-resistant depression. Biological psychiatry. Apr 15 2003;53(8):649-659.

142. McIntyre RS, Filteau MJ, Martin L, et al. Treatment-resistant depression: definitions, review of the evidence, and algorithmic approach. Journal of affective disorders. Mar 2014;156:1-7.

143. Dobscha SK, Morasco BJ, Duckart JP, Macey T, Deyo RA. Correlates of prescription opioid initiation and long-term opioid use in veterans with persistent pain. The Clinical journal of pain. 2013;29(2):102.

144. Scherrer JF, Salas J, Copeland LA, et al. Increased risk of depression recurrence after initiation of prescription opioids in noncancer pain patients. The Journal of Pain. 2016;17(4):473-482.

145. Ballinger GA. Using generalized estimating equations for longitudinal data analysis. Organizational research methods. 2004;7(2):127-150.

146. Szmaragd C, Clarke $P$, Steele F. Subject specific and population average models for binary longitudinal data: a tutorial. Longitudinal and Life Course Studies. 2013;4(2):147165.

147. Thielke SM, Fan M-Y, Sullivan M, Unützer J. Pain limits the effectiveness of collaborative care for depression. The American Journal of Geriatric Psychiatry. 2007;15(8):699-707.

148. Fava M, Memisoglu A, Thase ME, et al. Opioid modulation with buprenorphine/samidorphan as adjunctive treatment for inadequate response to antidepressants: a randomized double-blind placebo-controlled trial. American Journal of Psychiatry. 2016;173(5):499-508.

149. Karp JF, Butters MA, Begley A, et al. Safety, tolerability, and clinical effect of low-dose buprenorphine for treatment-resistant depression in mid-life and older adults. The Journal of clinical psychiatry. 2014;75(8):e785.

150. Lovejoy TI, Morasco BJ, Demidenko MI, Meath TH, Frank JW, Dobscha SK. Reasons for discontinuation of long-term opioid therapy in patients with and without substance use disorders. Pain. Mar 2017;158(3):526-534.

151. Berna C, Kulich RJ, Rathmell JP. Tapering Long-term Opioid Therapy in Chronic Noncancer Pain: Evidence and Recommendations for Everyday Practice. Mayo Clinic Proceedings. 2015;90(6):828-842.

152. Haffajee RL, Frank RG. Making the Opioid Public Health Emergency Effective. JAMA psychiatry. 2018;75(8):767-768. 
153. Edlund MJ, Steffick D, Hudson T, Harris KM, Sullivan M. Risk factors for clinically recognized opioid abuse and dependence among veterans using opioids for chronic noncancer pain. Pain. 2007;129(3):355-362.

154. Ciechanowski $P$, Wagner E, Schmaling K, et al. Community-Integrated Home-Based Depression Treatment in Older AdultsA Randomized Controlled Trial. JAMA. 2004;291(13):1569-1577.

155. Croghan TW, Brown JD. Integrating mental health treatment into the patient centered medical home. Agency for Healthcare Research and Quality Rockville, MD; 2010.

156. Barrett $K$, Chang YP. Behavioral interventions targeting chronic pain, depression, and substance use disorder in primary care. Journal of Nursing Scholarship. 2016;48(4):345353.

157. Institute of Medicine (US) Committee on Advancing Pain Research, Care, and Education. Relieving Pain in America: A Blueprint for Transforming Prevention, Care, Education, and Research. Washington (DC): National Academies Press (US); 2011.

158. Thase ME. New medications for treatment-resistant depression: a brief review of recent developments. CNS spectrums. 2017;22(S1):39-48.

159. Medicare Cf, Services M. Episode payment models: general information. 2016.

160. Gunaseelan V, Kenney B, Lee JS-J, Hu HM. Invited commentary: databases for surgical health services research: Clinformatics Data Mart. Elsevier; 2018.

\section{Appendices}




\section{Appendix 7.1. ICD-9/10 diagnosis codes used to identify CNPC, MDD and other chronic conditions}

\begin{tabular}{|c|c|c|}
\hline Condition & ICD-9 code set used & ICD-10 code set used \\
\hline $\begin{array}{l}\text { Major Depressive } \\
\text { Disorders }\end{array}$ & $\begin{array}{l}\text { 296.2x - dysthymic disorder (major } \\
\text { depressive disorder-single episode) } \\
296.3 x \text { - dysthymic disorder (major } \\
\text { depressive disorder, recurrent } \\
\text { episode) } \\
\text { 309.0x - prolonged depressive } \\
\text { reaction } \\
309.1 x \text { - prolonged depressive } \\
\text { reaction } \\
300.4 x \text { - Adjustment disorder with } \\
\text { depressed mood } \\
311 . x x \text { - depressive disorder, not } \\
\text { elsewhere classified }\end{array}$ & $\begin{array}{l}\text { F320 - Major depressive disorder, single } \\
\text { episode, mild } \\
\text { F321 - Major depressive disorder, single } \\
\text { episode, moderate } \\
\text { F322 - Major depressv disord, single epsd, } \\
\text { sev w/o psych features } \\
\text { F323 - Major depressv disord, single epsd, } \\
\text { severe w psych features } \\
\text { F324 - Major depressv disorder, single } \\
\text { episode, in partial remission } \\
\text { F325 - Major depressive disorder, single } \\
\text { episode, in full remission } \\
\text { F328- Other depressive episodes } \\
\text { F3289 - Other specified depressive episodes } \\
\text { F329 -Major depressive disorder, single } \\
\text { episode, unspecified } \\
\text { F330 - Major depressive disorder, recurrent, } \\
\text { mild } \\
\text { F331 -Major depressive disorder, recurrent, } \\
\text { moderate } \\
\text { F332 - Major depressive disorder, recurrent } \\
\text { severe w/o psych features } \\
\text { F333 - Major depressive disorder, recurrent, } \\
\text { severe w psych symptoms } \\
\text { F3340- Major depressive disorder, recurrent, } \\
\text { in remission, unspecified } \\
\text { F3341 - Major depressive disorder, } \\
\text { recurrent, in partial remission } \\
\text { F3342 -Major depressive disorder, recurrent, } \\
\text { in full remission } \\
\text { F338 - Other recurrent depressive disorders } \\
\text { F339 - Major depressive disorder, recurrent, } \\
\text { unspecified } \\
\text { F341 - persistent depression } \\
\text { F432 - Stress induced depression }\end{array}$ \\
\hline $\begin{array}{l}\text { schizophrenia, } \\
\text { schizoaffective, } \\
\text { other psychotic } \\
\text { disorders related to } \\
\text { schizophrenia, } \\
\text { manic depression } \\
\text { or bipolar disorders }\end{array}$ & $\begin{array}{l}\text { 293.81, 293.82, 295,296.0, 296.1, } \\
296.4,296.5,296.6,296.7,296.8 \\
296.9,298\end{array}$ & $\begin{array}{l}\text { F20, F06.0, F06.2, F25, F21, F22, F23, F24, } \\
\text { F25.0, F25.1, F25.8, F28, F29, F31, }\end{array}$ \\
\hline Back/neck pain & $\begin{array}{l}720.0,721.3 x-721.9 x, 722.2 x \\
722.30,722.70,722.80,722.90 \\
722.32,722.72,722.82,722.92\end{array}$ & $\begin{array}{l}\text { M45.9, M46.1, M49.80, M40, M46.90 } \\
\text { M47, M48, M50.20, M50.30, M51 } \\
\text { M50.00, M96.1, M961, M46.4, M50.80 }\end{array}$ \\
\hline
\end{tabular}




\begin{tabular}{|c|c|c|}
\hline & $\begin{array}{l}\text { 722.33, 722.73, 722.83, 722.93, } \\
\text { 724.xx, 737.1, 737.3, 738.4, 738.5, } \\
\text { 739.2, 739.3, 739.4, 756.10, 756.11, } \\
\text { 756.12, 756. 13, 756.19, 805.4, } \\
\text { 805.8, 839.2, 839.42, 846, 846.0, } \\
\text { 847.1, 847.3, 847.2, 847.9 } \\
\text { 721.0x, 721.1x, 722.0x, 722.31, } \\
\text { 722.71, 722.81, 722.91, 723.XX, } \\
\text { 839.0, 839.1, 847.0 }\end{array}$ & $\begin{array}{l}\text { M50.90, M46.45, M48.02 M54.2, M53.0, } \\
\text { M53.1, M54.12, M54.13 M43.6, M54.02, } \\
\text { M67.88, M53.82, M48.00, M4804, M48.06 } \\
\text { M48.08, M54.6, M54.5, M54.30, M54.14 } \\
\text { M54.15, M54.16, M54.17, M54.89, M54.9 } \\
\text { M43.2, M53.2, M53.3, M54.08, M43.8X9 } \\
\text { M53.9, M96.2, M96.4Q7649, } \\
\text { Q76.2, Q76.49, Q76.419, Q76.49, S32009A } \\
\text { S12.9XXA, S32.009A, S32.10XA, } \\
\text { S32.2XXA } \\
\text { S338.XXA, S33.6XXA, S33.9XXA, } \\
\text { S13.4XXA } \\
\text { S13.8XXA, S23.3XXA, S23.3XXA, } \\
\text { S23.8XXA } \\
\text { S33.5XXA, S33.8XXA, S33.8XXA, } \\
\text { S23.9XXA }\end{array}$ \\
\hline Headache/migraine & $\begin{array}{l}339.05,339.09,339.10,339.11, \\
339.12,339.20,339.3,339.41, \\
339.42,339.43,339.44,339.81, \\
339.82,339.83,339.84,339.85, \\
339.89, \geq 346 \text { and }<347, \\
784.0,307.81\end{array}$ & $\begin{array}{l}\text { G44.85, G44.89, G43.109, G43.119, } \\
\text { G43.101 } \\
\text { G43.111, G43.009, G43.019, G43.001, } \\
\text { G43.011, } \\
\text { G43.809, G43.A0, G43.B0, G43.C0, } \\
\text { G43.D0, } \\
\text { G43.819, G43.A1, G43.B1, G43.C1, } \\
\text { G43.D1, } \\
\text { G43.801, G43.811, G43.409, G43.419, } \\
\text { G43.401 } \\
\text { G43.411, G43.829, G43.839, G43.821, } \\
\text { G43.831, G43.509, G43.519, G43.501 } \\
\text { G43.511, G43.709, G43.719, G43.701 } \\
\text { G43.711, G43.809, G43.819, G43.801 } \\
\text { G43.811, G43.909, G43.919, G43.901, } \\
\text { G43.911, } \\
\text { G44.1, R51, G44.209 }\end{array}$ \\
\hline Arthritis/joint pain & $\begin{array}{l}710,711,713,714,715,716,717, \\
718,719,725,726,727,728,729,730- \\
739\end{array}$ & $\begin{array}{l}\text { M32.10, M34.0, M341, M34.9, M35.0 } \\
\text { M35.01, M00.10, M00-M02 } \\
\text { M36.2, M36.3, M12.2, M12.3, M12.4, } \\
\text { M12.80, M12.80, M05, } \\
\text { M06, M08, M12.0, M33.20, M45, M48.8, } \\
\text { M15, M16, M17,M18, M19, M12.1, } \\
\text { M12.1,M12.8, M12.9, M13.0,M13.1, } \\
\text { M13.8,M23.2, M23.3,M23.4, M23.5, M23.6, } \\
\text { M23.8,M23.9, M24,M43.4, M25, } \\
\text { M79.64,R29.89, R29.4, R26.2, M35.3, } \\
\text { M65,M61, M62, M63, M66, M67, M70- } \\
\text { M79,M85, M86,M87, M88.9, M89.00, } \\
\text { M899, M89.6, M89.7, M90-M93,M94.0, } \\
\text { M94.9 }\end{array}$ \\
\hline $\begin{array}{l}\text { Neuropathic } \\
\text { pain/other chronic } \\
\text { pain }\end{array}$ & $\begin{array}{l}\text { 357, 337.0, 356.0, 356.2, 356.4, } \\
\text { 356.9, 357.2, 357.3, 531.3, 723.4, } \\
727.2,338\end{array}$ & $\begin{array}{l}\text { G60, G61.9, G61.0, G81.8, G62.0, G62.2, } \\
\text { G63, E08.42, E09.42, E10.42, G89 }\end{array}$ \\
\hline
\end{tabular}


Appendix 7.2. Chapter 2. Study design for predicting treatment resistant depression among adults with CNPC and newly diagnosed MDD

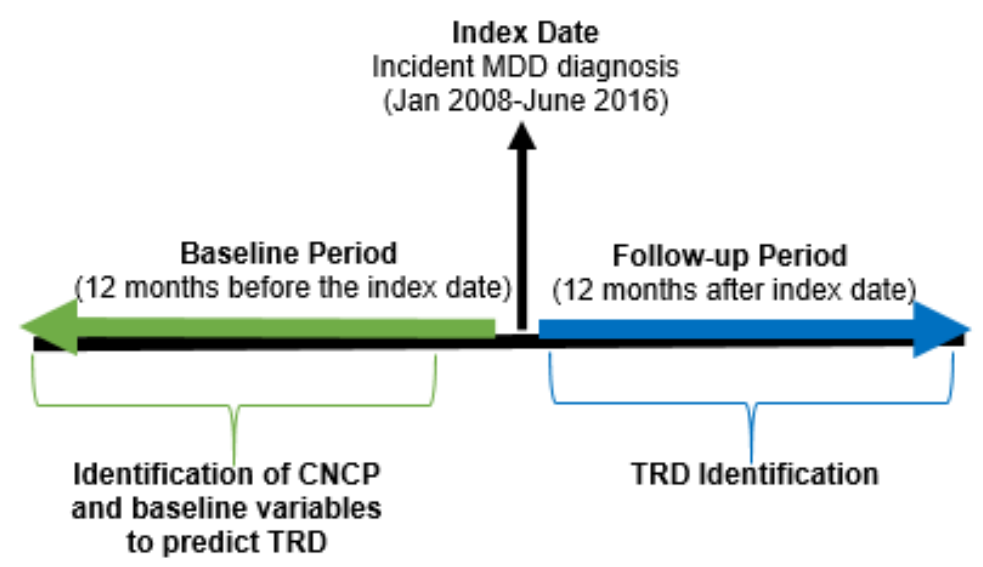




\section{Appendix 7.3. Chapter 2. Representation of development and testing of machine learning model}

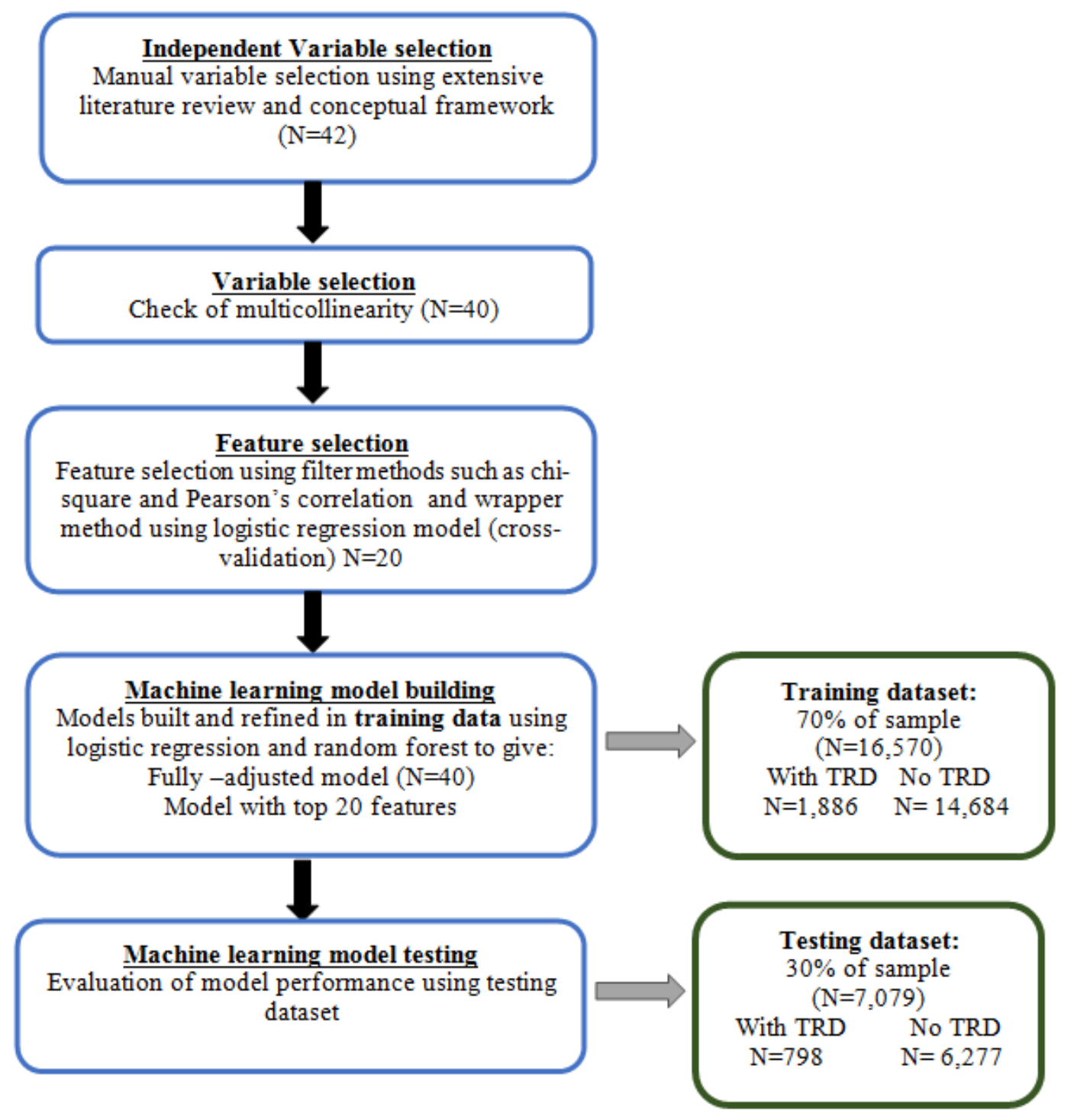


Appendix 7.4. Chapter 2. Components of the Algorithm for identification of patients with TRD

\begin{tabular}{|l|l|l|}
\hline Component of TRD algorithm and the assigned score & $\mathbf{N}$ & Frequency \\
\hline $\begin{array}{l}\text { At least } 1 \text { adequate trial of antidepressants, i.e. } \geq 2 \text { fills of same } \\
\text { antidepressant medication (1 point) }\end{array}$ & 19,740 & $83.48 \%$ \\
\hline $\begin{array}{l}\text { At least one extended duration, i.e. at least 3 fills of each antidepressant } \\
\text { medication (0.5 point) }\end{array}$ & 17,557 & $74.25 \%$ \\
\hline Upward titration in dose (0.5 point) & 5,537 & $23.42 \%$ \\
\hline $\begin{array}{l}\text { Augmentation with atypical antipsychotics/mood stabilizer/thyroid hormone } \\
\text { (0.5 point for each augmentation strategy) }\end{array}$ & 782 & $3.31 \%$ \\
-Augmentation with atypical antipsychotics^ & 645 & $2.73 \%$ \\
-With mood stabilizers (carbamazepine, lamotrigine, lithium, valproic acid) & 181 & $0.77 \%$ \\
-With thyroid hormone (liothyronine) & 21 & $0.09 \%$ \\
\hline ECT use -considered directly as having TRD (4 points) & 11 & $0.005 \%$ \\
\hline TRD (> 3 points) & $\mathbf{2 , 6 8 4}$ & $\mathbf{1 1 . 3 5 \%}$ \\
\hline No TRD & $\mathbf{2 0 , 9 6 1}$ & $\mathbf{8 8 . 6 5 \%}$ \\
\hline $\begin{array}{l}\text { Note: Based on 23,645 adults with CNPC and newly diagnosed episode of MDD using antidepressant } \\
\text { medications, with continuous enrollment for 2 years in a commercial insurance plan. Individual }\end{array}$ \\
$\begin{array}{l}\text { components of TRD criteria will not add to 11.35\% because an individual may satisfy one component of } \\
\text { the algorithm while failing to satisfy another component of the algorithm. } \\
\text { ^ Medication names included in the class of atypical antipsychotics include aripiprazole, asenapine } \\
\text { maleate, brexpiprazole, cariprazine, clozapine, lurasidone, olanzapine, paliperidone, paliperidone } \\
\text { palmitate, quetiapine fumarate, risperidone, risperidone microspheres, and ziprasidone }\end{array}$ \\
\hline
\end{tabular}

Appendix 7.5. Chapter 2. Performance measures for the prediction models

Model

Training set

Logistic Regression

Fully adjusted model $(n=40)$

Top 20 features

\section{Random forest}

Fully adjusted model $(n=40)$

Top 20 features

\section{AUC Sensitivity Specificity}

0.711

0.704

0.716

0.7
0.482

0.48

0.52

0.4
0.811

0.799

0.9

1
Out of bag error

Testing set

\section{Logistic Regression}

Fully adjusted model $(n=40)$

Top 20 features

\section{Random forest}

Fully adjusted model $(n=40)$
0.708

0.705

0.704
0.576

0.572

0.48
N/A

N/A

$11.42 \%$

$11.51 \%$ 


\begin{tabular}{|c|c|c|c|c|c|c|}
\hline \multicolumn{7}{|c|}{$\begin{array}{l}\text { Appendix 7.6. Chapter 3. Baseline patient characteristics of adults with CNPC and Newly } \\
\text { Diagnosed MDD by TRD status before and after Inversed Probability of Treatment } \\
\text { Weighting (IPTW), Optum Clinformatics DataMart, 2007-2017 }\end{array}$} \\
\hline & $\begin{array}{c}\text { Patients } \\
\text { classified } \\
\text { with TRD } \\
(\mathrm{N}=\mathbf{2 , 1 4 7 )}\end{array}$ & $\begin{array}{c}\text { Patients } \\
\text { without } \\
\text { TRD } \\
(\mathbf{N}=\mathbf{1 9 , 0 3 3 )}\end{array}$ & & $\begin{array}{l}\text { Patients } \\
\text { classified } \\
\text { with } \\
\text { TRD } \\
(\mathrm{N}=2,143)\end{array}$ & $\begin{array}{l}\text { Patients } \\
\text { without } \\
\text { TRD } \\
(\mathrm{N}=19,034)\end{array}$ & \\
\hline & $\%$ & $\%$ & P value & Wt. \% & Wt. \% & $P$ value \\
\hline \multicolumn{7}{|l|}{ Characteristics } \\
\hline Index Year & & & $<0.001$ & & & 0.870 \\
\hline $2008-2010$ & 23.1 & 23.3 & & 23.4 & 23.3 & \\
\hline 2011-2013 & 38.7 & 33.3 & & 34.3 & 33.8 & \\
\hline 2014-2016 & 38.1 & 43.5 & & 42.3 & 42.9 & \\
\hline \multicolumn{7}{|c|}{ Predisposing factors } \\
\hline Sex & & & $<0.001$ & & & 0.705 \\
\hline Female & 77.0 & 73.3 & & 74.1 & 73.7 & \\
\hline Age group & & & $<0.001$ & & & 0.732 \\
\hline $18-34$ years & 13.6 & 13.1 & & 12.7 & 13.1 & \\
\hline $35-44$ years & 16.8 & 14.5 & & 14.3 & 14.7 & \\
\hline $45-54$ years & 23.1 & 19.4 & & 19.7 & 19.8 & \\
\hline $55-64$ years & 20.9 & 19.1 & & 18.8 & 19.3 & \\
\hline$\geq 65$ years & 25.6 & 33.9 & & 34.5 & 33.1 & \\
\hline \multicolumn{7}{|c|}{ Enabling factors } \\
\hline Medicare Advantage & & & 0.106 & & & 0.366 \\
\hline $\begin{array}{l}\text { Commercial } \\
\text { Medicare }\end{array}$ & 60.5 & 58.7 & & 57.8 & 58.9 & \\
\hline Advantage & 39.5 & 41.3 & & 42.2 & 41.1 & \\
\hline Insurance plan type & & & 0.269 & & & 0.742 \\
\hline HMO & 30.8 & 32.2 & & 32.9 & 32.1 & \\
\hline $\mathrm{PPO} / \mathrm{POS}$ & 48.5 & 46.8 & & 46.2 & 46.9 & \\
\hline Other & 20.7 & 21.0 & & 20.9 & 21.0 & \\
\hline \multicolumn{7}{|c|}{ Need factors } \\
\hline No. of CNPC a & & & $<0.001$ & & & 0.844 \\
\hline 1 & 18.7 & 23.2 & & 23.2 & 22.8 & \\
\hline 2 & 40.7 & 45.0 & & 43.9 & 44.5 & \\
\hline$\geq 3$ & 40.6 & 31.8 & & 32.9 & 32.7 & \\
\hline \multicolumn{3}{|c|}{ Number of physical chronic conditions ${ }^{b}$} & 0.893 & & & 0.112 \\
\hline 0 & 37.6 & 37.4 & & 36.6 & 37.4 & \\
\hline
\end{tabular}




\begin{tabular}{|c|c|c|c|c|c|c|}
\hline 1 to 2 & 36.2 & 36.0 & & 34.7 & 36.0 & \\
\hline$\geq 3$ & 26.2 & 26.7 & & 28.6 & 26.5 & \\
\hline Anxiety disorders & & & $<0.001$ & & & 0.527 \\
\hline Yes & 55.7 & 36.1 & & 38.8 & 38.1 & \\
\hline No & 44.3 & 63.9 & & 61.2 & 61.9 & \\
\hline Sleep disorders & & & $<0.001$ & & & 0.970 \\
\hline Yes & 33.6 & 23.7 & & 24.6 & 24.7 & \\
\hline No & 66.4 & 76.3 & & 75.4 & 75.3 & \\
\hline Obesity diagnosis & & & 0.070 & & & 0.860 \\
\hline Yes & 17.8 & 16.3 & & 16.3 & 16.5 & \\
\hline No & 82.2 & 83.7 & & 83.7 & 83.5 & \\
\hline Tobacco use disorders & & & $<0.001$ & & & 0.910 \\
\hline Yes & 14.9 & 11.1 & & 11.4 & 11.5 & \\
\hline No & 85.1 & 88.9 & & 88.6 & 88.5 & \\
\hline Drug/alcohol use disorder & & & $<0.001$ & & & 0.830 \\
\hline Yes & 15.9 & 8.1 & & 8.8 & 8.9 & \\
\hline No & 84.1 & 91.9 & & 91.2 & 91.1 & \\
\hline \multicolumn{7}{|c|}{ Environmental factors } \\
\hline Census region of residence & & & 0.156 & & & 0.870 \\
\hline Northeast & 10.1 & 8.9 & & 9.6 & 9.0 & \\
\hline Midwest & 24.9 & 24.3 & & 24.0 & 24.3 & \\
\hline South & 40.3 & 40.7 & & 40.4 & 40.6 & \\
\hline West & 24.6 & 26.1 & & 26.0 & 26.0 & \\
\hline
\end{tabular}

Abbreviations: MDD: Major Depressive Disorder, TRD: Treatment- resistant depression, CNPC: Chronic non-cancer pain conditions, HMO: Health Maintenance Organization, PPO: Preferred provider organization, POS: Point of service, other plan type includes fee-for-service, indemnity plans, EPO (exclusive provider organization), GPO (Group purchasing organization), IPP(individual program plan), and other plans, Wt.: Weighted

Note: This sample includes adult patients with CNPC and newly diagnosed MDD who were continuously enrolled in the medical and pharmacy obtained from the de-identified Optum Clinformatics DataMart. IPTW weights were derived from a multivariate logistic regression on TRD status using the above predisposing, enabling, need and environmental factors

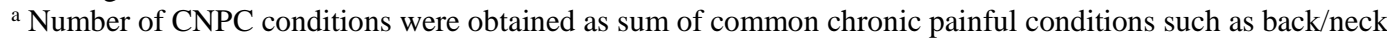
pain, headache/migraine, arthritis/joint pain, and neuropathic pain/other chronic pain conditions.

${ }^{b}$ Physical chronic conditions examined included conditions other than CNPC conditions such as asthma, coronary artery disease, cardiac arrhythmias, congestive heart failure, chronic kidney disease, chronic obstructive pulmonary disease, diabetes, hypertension, hyperlipidemia, osteoporosis, and stroke. 


\begin{tabular}{|c|c|c|c|c|}
\hline \multicolumn{5}{|c|}{$\begin{array}{l}\text { Appendix 7.7. Chapter 3. Mean Annual IPTW Adjusted and Unadjusted All-Cause and MDD-Related Costs Among } \\
\text { Adults with CNPC and Newly Diagnosed MDD } \\
\text { Optum Clinformatics DataMart, 2007-2017 }\end{array}$} \\
\hline & $\begin{array}{c}\text { Patients } \\
\text { classified with } \\
\text { TRD }\end{array}$ & $\begin{array}{c}\text { Patients } \\
\text { without TRD }\end{array}$ & $\begin{array}{l}\text { Adjusted Incremental costs, } \\
\text { Model } 1^{\text {a }}\end{array}$ & $\begin{array}{l}\text { Adjusted Incremental } \\
\text { Costs, Model } 2^{\text {b }}\end{array}$ \\
\hline Outcomes & $\begin{array}{l}\text { Unadjusted } \\
\text { Mean (SD), \$ }\end{array}$ & $\begin{array}{l}\text { Unadjusted } \\
\text { Mean (SD), \$ }\end{array}$ & $\begin{array}{c}\text { Mean } \$ \\
(95 \% \text { CI }) ; P \text { value }\end{array}$ & $\begin{array}{c}\text { Mean } \$ \\
(95 \% \text { CI }) ; \text { P value }\end{array}$ \\
\hline $\begin{array}{l}\text { All-cause healthcar } \\
\text { Total }\end{array}$ & $\begin{array}{c}\text { ts (2018 US\$) } \\
19,670 \\
(41,965)\end{array}$ & $\begin{array}{c}14,886 \\
(42,737)\end{array}$ & $\begin{array}{c}3,471 \\
(3,436-3,504) ;<0.001\end{array}$ & $\begin{array}{c}2,039 \\
2,017-2,058) ;<0.001\end{array}$ \\
\hline $\begin{array}{l}\text { Prescription drug } \\
\text { costs }\end{array}$ & $\begin{array}{c}5,909 \\
(9,281)\end{array}$ & $\begin{array}{l}3,953 \\
(9,067)\end{array}$ & $\begin{array}{c}1,362 \\
(1,350-1,375) ;<0.001\end{array}$ & $\begin{array}{c}773 \\
(765-782) ;<0.001\end{array}$ \\
\hline Inpatient & $\begin{array}{c}7,285 \\
(34,122)\end{array}$ & $\begin{array}{c}5,720 \\
(34,896)\end{array}$ & $\begin{array}{c}1,240 \\
(1,226-1,256) ; 0.011\end{array}$ & $\begin{array}{c}903 \\
(892-915) ; 0.148\end{array}$ \\
\hline Outpatient & $\begin{array}{c}5,024 \\
(16,815)\end{array}$ & $\begin{array}{c}4,046 \\
(17,504)\end{array}$ & $\begin{array}{c}764 \\
(758-770) ; 0.008\end{array}$ & $\begin{array}{c}637 \\
(637-643) ; 0.012\end{array}$ \\
\hline ED & $\begin{array}{c}1,454 \\
(4,237)\end{array}$ & $\begin{array}{l}1,169 \\
(4,036)\end{array}$ & $\begin{array}{c}231 \\
(229-232) ; 0.002\end{array}$ & $\begin{array}{c}192 \\
(190-193) ; 0.011\end{array}$ \\
\hline \multicolumn{5}{|c|}{$\begin{array}{l}\text { MDD-related healthcare costs (2018 } \\
\text { US\$) }\end{array}$} \\
\hline Total medical costs & $\begin{array}{l}1,009 \\
(5,067)\end{array}$ & $\begin{array}{c}480 \\
(3,774)\end{array}$ & $\begin{array}{c}392 \\
(387-397) ;<0.001\end{array}$ & $\begin{array}{c}298 \\
(293-303) ;<0.001\end{array}$ \\
\hline Inpatient & $\begin{array}{c}434 \\
(3,842)\end{array}$ & $\begin{array}{c}172 \\
(2,880)\end{array}$ & $\begin{array}{c}267 \\
(263-271) ;<0.001\end{array}$ & $\begin{array}{c}224 \\
(220-228) ;<0.001\end{array}$ \\
\hline Outpatient & $\begin{array}{c}413 \\
(2,610)\end{array}$ & $\begin{array}{c}216 \\
(1,855)\end{array}$ & $\begin{array}{c}97 \\
(95-99) ;<0.001\end{array}$ & $\begin{array}{c}69 \\
(68-71) ;<.001\end{array}$ \\
\hline ED & $\begin{array}{c}162 \\
(1,075)\end{array}$ & $\begin{array}{c}91 \\
(873)\end{array}$ & $\begin{array}{c}14 \\
(13-15) ; 0.002\end{array}$ & $\begin{array}{c}12 \\
(11-13) ; 0.005\end{array}$ \\
\hline \multicolumn{5}{|c|}{$\begin{array}{l}\text { Abbreviations: MDD: Major Depressive Disorder, TRD: Treatment- resistant depression, CNPC: Chronic non-cancer pain conditions, } \\
\text { SD: Standard deviation; ED: Emergency Department } \\
\text { Note: The sample includes all eligible adults with CNPC and newly diagnosed MDD who are treated with antidepressants and were } \\
\text { continuously enrolled throughout the study observation period (N=21,180) from the de-identified Optum Clinformatics DataMart. } \\
\text { All the costs were standardized to } 2018 \text { US dollar. Total all-cause healthcare costs included the standardized costs for all healthcare } \\
\text { medical and pharmacy services paid by payers, including those for outpatient visits, inpatient stays, ED visits, and prescription } \\
\text { medications. Total MDD-related medical costs included costs for medical- related services including inpatient visits, outpatient and ED } \\
\text { visits. Total MDD-related costs were identified based on primary/secondary diagnoses of MDD. } \\
\text { Individual weights based on inverse probability of treatment weighting (IPTW) have been used for these analyses. } \\
\text { Unadjusted incremental costs and their corresponding P value and } 95 \% \text { CIs have been obtained using ordinary least square regressions. } \\
\text { a Incremental costs from Model } 1 \text { were measured using counterfactual recycled predictions performed using estimates obtained from a 2- } \\
\text { part GLM model with log link and gamma distribution which adjusted for baseline factors: predisposing factors (age, sex), enabling } \\
\text { factors (Medicare Advantage enrollment, type of insurance plan), need based factors (number of physical chronic conditions, number of } \\
\text { CNPC, presence of anxiety disorders, sleep disorders, obesity, and baseline medication use (polypharmacy, NSAID, opioid and } \\
\text { benzodiazepine use), and environmental factors (region). } \\
\text { b Incremental costs from Model } 2 \text { were measured using counterfactual recycled predictions performed using estimates obtained from a 2- } \\
\text { part GLM model with log link and gamma distribution which adjusted for all baseline factors mentioned in model } 1 \text { and healthcare costs. } \\
\text { a,b All P values and their confidence intervals were obtained using nonparametric bootstrap procedure (N=500) on the predicted values } \\
\text { obtained from recycled predictions. }\end{array}$} \\
\hline
\end{tabular}




\begin{tabular}{|c|c|c|c|c|c|}
\hline \multicolumn{6}{|c|}{$\begin{array}{c}\text { Appendix 7.8. Chapter 3. Mean Annual Adjusted and Unadjusted All-Cause and MDD-Related } \\
\text { Utilization Among Adults with CNPC and Newly Diagnosed MDD Treated with Antidepressants by TRD } \\
\text { status } \\
\text { Optum Clinformatics DataMart, 2007-2017 }\end{array}$} \\
\hline \multirow[b]{2}{*}{$\begin{array}{l}\text { Healthcare } \\
\text { resource } \\
\text { utilization }\end{array}$} & \multicolumn{2}{|c|}{$\begin{array}{c}\text { Patients classified with } \\
\text { TRD }\end{array}$} & \multicolumn{2}{|c|}{ Patients without TRD } & \multirow[b]{2}{*}{$\begin{array}{c}\text { Adjusted IRR }{ }^{\text {a }} \\
(95 \% \text { CI }) ; \text { P value }\end{array}$} \\
\hline & $\begin{array}{l}\mathrm{N} \\
(\% \text { with } \\
\text { visit })\end{array}$ & $\begin{array}{l}\text { Mean (number } \\
\text { of visits), (SD) }\end{array}$ & $\begin{array}{l}\mathrm{N} \\
(\% \text { with } \\
\text { visit) }\end{array}$ & $\begin{array}{l}\text { Mean } \\
\text { (number of } \\
\text { visits), (SD) }\end{array}$ & \\
\hline \multicolumn{6}{|l|}{ All-cause } \\
\hline Inpatient & $480(22.4 \%)$ & $0.47(1.28)$ & $3,023(15.9 \%)$ & $0.30(0.97)$ & $\begin{array}{c}1.30 \\
(1.14-1.47) ;<0.001\end{array}$ \\
\hline Outpatient & $1,636(76.2)$ & $8.04(15.80)$ & $12,953(68.1 \%)$ & $6.72(16.82)$ & $\begin{array}{c}1.24 \\
(1.15-1.34) ;<0.001\end{array}$ \\
\hline $\mathrm{ED}$ & 857 (39.9\%) & $1.06(2.40)$ & $5,859(30.8 \%)$ & $0.71(1.94)$ & $\begin{array}{c}1.21 \\
(1.10-1.34) ;<0.001\end{array}$ \\
\hline \multicolumn{6}{|c|}{ MDD-related } \\
\hline Inpatient & $77(3.6 \%)$ & $0.05(0.26)$ & $198(1.0 \%)$ & $0.01(0.12)$ & $\begin{array}{c}3.00 \\
(2.15-4.18) ;<0.001\end{array}$ \\
\hline Outpatient & $167(7.8 \%)$ & $3.86(6.88)$ & $625(3.3 \%)$ & $2.41(4.53)$ & $\begin{array}{c}2.95 \\
(2.03-4.29) ;<0.001\end{array}$ \\
\hline ED & $49(2.3 \%)$ & $0.03(0.23)$ & $154(0.8 \%)$ & $0.01(0.13)$ & $\begin{array}{c}2.23 \\
(1.52-3.29) ;<0.001\end{array}$ \\
\hline \multicolumn{6}{|c|}{$\begin{array}{l}\text { Abbreviations: MDD: Major Depressive Disorder, TRD: Treatment- resistant depression, CNPC: Chronic non-cancer pain conditions, } \\
\text { SD: Standard deviation; ED: Emergency Department; CI: Confidence Interval; IRR: Incidence rate ratio } \\
\text { Note: The sample includes all eligible adults with CNPC and newly diagnosed MDD who are treated with antidepressants and were } \\
\text { continuously enrolled throughout the study observation period (N=21,180) from the de-identified Optum Clinformatics DataMart. } \\
\text { MDD-related costs were identified based on primary/secondary diagnoses of MDD. } \\
\text { a Adjusted IRRs and their corresponding 95\% CIs and P values were obtained using a generalized linear model (GLM) with a negative } \\
\text { binomial distribution (based on the results of the over-dispersion test). The model adjusted for baseline predisposing factors (age, sex), } \\
\text { enabling factors (Medicare Advantage enrollment, type of insurance plan), need based factors (number of physical chronic conditions, } \\
\text { number of CNPC, presence of anxiety disorders, sleep disorders, obesity, and baseline medication use (polypharmacy, NSAID, opioid } \\
\text { and benzodiazepine use), environmental factor (region), and baseline healthcare utilization. }\end{array}$} \\
\hline
\end{tabular}




\section{Appendix 7.9. Chapter 3. Unadjusted and Adjusted All-Cause Total and MDD-related Total Costs Over Time Among Adults with CNPC and Newly Diagnosed MDD Treated with Antidepressants by TRD status Optum Clinformatics DataMart, 2007-2017}

\begin{tabular}{|c|c|c|c|c|c|c|}
\hline \multirow[t]{2}{*}{$\begin{array}{l}\text { Costs/ } \\
\text { time }\end{array}$} & \multicolumn{2}{|c|}{$\begin{array}{l}\text { Patients classified } \\
\text { with TRD }\end{array}$} & \multicolumn{2}{|c|}{$\begin{array}{c}\text { Patients without } \\
\text { TRD }\end{array}$} & \multirow{2}{*}{$\begin{array}{c}\text { Unadjusted weighted } \\
\text { cost difference } \\
\text { between TRD and } \\
\text { non-TRD groups }{ }^{\text {a }}\end{array}$} & \multirow[t]{2}{*}{$\begin{array}{l}\text { Adjusted weighted cost } \\
\text { difference between TRD } \\
\text { and non-TRD groups }\end{array}$} \\
\hline & Mean \$ & SD \$ & Mean \$ & SD \$ & & \\
\hline \multicolumn{5}{|c|}{$\begin{array}{l}\text { All-cause total healthcare costs ( } 2018 \\
\text { US\$) }\end{array}$} & $\begin{array}{l}\beta(\text { time })=-0.023 \\
P \text { Value }<0.001 \\
\beta(\text { TRD })=0.35 \\
P \text { Value }<0.001\end{array}$ & $\begin{array}{l}\beta(\text { time })=-0.017 \\
P \text { Value }=0.005 \\
\beta(\text { TRD })=0.31 \\
P \text { Value }<0.001\end{array}$ \\
\hline Time 1 & $8,268.9$ & $20,831.6$ & $5,195.9$ & $18,404.8$ & 2389.33 & 1976.19 \\
\hline Time 2 & $6,876.9$ & $15,536.1$ & $5,195.7$ & $17,152.4$ & 2282.78 & 1881.69 \\
\hline Time 3 & $7,967.2$ & $18,791.7$ & $6,191.7$ & $27,304.4$ & 2180.22 & 1789.21 \\
\hline Time 4 & $7,122.5$ & $15,316.3$ & $5,074.2$ & $26,925.9$ & 2081.52 & 1698.7 \\
\hline Time 5 & $6,998.8$ & $21,185.4$ & $4,654.0$ & $17,284.4$ & 1986.54 & 1610.12 \\
\hline \multirow[t]{2}{*}{ Time 6} & $6,808.9$ & $18,199.2$ & $4,914.9$ & $17,037.6$ & 1895.15 & 1523.45 \\
\hline & & & & & $\begin{array}{c}\text { Average difference = } \\
\mathbf{2 1 3 5 . 9 2} \\
\end{array}$ & $\begin{array}{c}\text { Average difference = } \\
\mathbf{1 7 4 6 . 5 6}\end{array}$ \\
\hline \multicolumn{5}{|c|}{$\begin{array}{l}\text { MDD -related total medical costs ( } 2018 \\
\text { US\$) }\end{array}$} & \multicolumn{2}{|l|}{$\begin{array}{l}\beta(\text { time })=-0.12 \\
P \text { Value }=0.007 \\
\beta(\text { TRD })=1.37 \\
P \text { Value }<0.001\end{array}$} \\
\hline Time 1 & 538.5 & $5,787.4$ & 83.0 & $3,548.9$ & \multicolumn{2}{|l|}{436.16} \\
\hline Time 2 & 339.6 & $3,484.5$ & 95.3 & $1,657.5$ & 335.34 & 365.81 \\
\hline Time 3 & 524.0 & $3,347.7$ & 254.3 & $4,956.6$ & 256.49 & 294.51 \\
\hline Time 4 & 348.4 & $3,944.7$ & 87.7 & $2,051.8$ & 194.97 & 218.63 \\
\hline Time 5 & 199.8 & $1,757.0$ & 67.2 & $1,784.9$ & 147.09 & 162.36 \\
\hline \multirow[t]{2}{*}{ Time 6} & 168.1 & $1,783.6$ & 63.2 & $1,287.7$ & 109.95 & 120.97 \\
\hline & & & & & $\begin{array}{c}\text { Average difference }= \\
246.67\end{array}$ & $\begin{array}{c}\text { Average difference }= \\
268.63\end{array}$ \\
\hline \multicolumn{7}{|c|}{$\begin{array}{l}\text { Abbreviations: MDD: Major Depressive Disorder, TRD: Treatment- resistant depression, CNPC: Chronic non-cancer pain conditions, SD: } \\
\text { Standard deviation; } \\
\text { Note: The sample includes all eligible adults with CNPC and newly diagnosed MDD who are treated with antidepressants and were } \\
\text { continuously enrolled throughout the study observation period (N=21,180) from the de-identified Optum Clinformatics DataMart. } \\
\text { All the costs were standardized to } 2018 \text { US dollar. Total all-cause healthcare costs included the standardized costs for all healthcare medical } \\
\text { and pharmacy services paid by payers, including those for outpatient visits, inpatient stays, ED visits, and prescription medications. Total } \\
\text { MDD-related medical costs included costs for medical- related services including inpatient visits, outpatient and ED visits. Total MDD- } \\
\text { related costs were identified based on primary/secondary diagnoses of MDD. } \\
\text { a Unadjusted weighted incremental costs were obtained using counterfactual recycled predictions performed using estimates from } \\
\text { Generalized Linear Mixed Models (GLMM) with log link and gamma distribution. } \\
\text { b Adjusted weighted incremental costs were obtained using counterfactual recycled predictions performed using estimates from Generalized } \\
\text { Linear Mixed Models (GLMM) with log link and gamma distribution. which adjusted for time invariant factors including predisposing } \\
\text { factors (age, sex), enabling factors (Medicare Advantage enrollment, type of insurance plan), need based factors (number of physical } \\
\text { chronic conditions, number of CNPC, presence of anxiety disorders, sleep disorders, obesity), environmental factors (region) and time } \\
\text { varying factors including polypharmacy, NSAIDs, opioid and benzodiazepine use }\end{array}$} \\
\hline
\end{tabular}




\begin{tabular}{|c|c|c|}
\hline \multicolumn{3}{|c|}{ Appendix 7.10. Chapter 4. Components of the Algorithm for identification of patients with TRD } \\
\hline Component of TRD algorithm and the assigned score & & $\begin{array}{r}\text { Frequency } \\
(\%)\end{array}$ \\
\hline $\begin{array}{l}\text { At least } 1 \text { adequate trial of antidepressants, i.e. } \geq 2 \text { fills of same antidepressant medication ( } 1 \\
\text { point) }\end{array}$ & 18,248 & $84.48 \%$ \\
\hline $\begin{array}{l}\text { At least one extended duration, i.e. at least } 3 \text { fills of each antidepressant medication }(0.5 \\
\text { point) }\end{array}$ & 16,341 & $75.65 \%$ \\
\hline Upward titration in dose ( 0.5 point $)$ & 5,124 & $23.72 \%$ \\
\hline $\begin{array}{l}\text { Augmentation with atypical antipsychotics/mood stabilizer/thyroid hormone ( } 0.5 \text { point for } \\
\text { each augmentation strategy) }\end{array}$ & 732 & $3.39 \%$ \\
\hline -Augmentation with atypical antipsychotic ${ }^{\wedge}$ & 606 & $2.81 \%$ \\
\hline -With mood stabilizers (carbamazepine, lamotrigine, lithium, valproic acid) & 166 & $0.77 \%$ \\
\hline -With thyroid hormone (liothyronine) & 20 & $0.09 \%$ \\
\hline ECT use -considered directly as having TRD & 11 & $0.0005 \%$ \\
\hline TRD (> 3 points) & 2,550 & $11.80 \%$ \\
\hline No TRD & 19,049 & $88.20 \%$ \\
\hline \multicolumn{3}{|c|}{$\begin{array}{l}\text { Note: Based on 21,599 adults with CNPC and newly diagnosed episode of MDD treated with antidepressants and are continuously enrolled } \\
\text { during the study observation period in a commercial insurance plan. Individual components of TRD criteria will not add to } 11.35 \% \text { because } \\
\text { an individual may satisfy one component of the algorithm while failing to satisfy another component of the algorithm. } \\
\wedge \text { Medication names included in the class of atypical antipsychotics include aripiprazole, asenapine maleate, brexpiprazole, cariprazine, } \\
\text { clozapine, lurasidone, olanzapine, paliperidone, paliperidone palmitate, quetiapine fumarate, risperidone, risperidone microspheres, and } \\
\text { ziprasidone }\end{array}$} \\
\hline
\end{tabular}




\begin{tabular}{|c|c|c|c|c|c|c|}
\hline \multicolumn{7}{|c|}{$\begin{array}{l}\text { Appendix 7.11. Chapter 4. Sample Characteristics by TRD Status among Adults } \\
\text { with CNPC and Newly Diagnosed MDD } \\
\text { Optum Clinformatics Real-World De-identified Data, 2007-2017 }\end{array}$} \\
\hline \multirow[b]{2}{*}{ Characteristics } & \multicolumn{2}{|c|}{$\begin{array}{c}\text { Patients } \\
\text { classified with } \\
\text { TRD }(N=2,550)\end{array}$} & \multicolumn{2}{|c|}{$\begin{array}{l}\text { Patients without } \\
\text { TRD }(\mathrm{N}=19,049)\end{array}$} & \multirow[b]{2}{*}{$\underset{\text { value }}{\mathbf{P}}$} & \multirow[b]{2}{*}{ Sig } \\
\hline & $\mathbf{N}$ & $\begin{array}{c}\text { Percent } \\
(\%)\end{array}$ & $\mathbf{N}$ & $\begin{array}{c}\text { Percent } \\
(\%)\end{array}$ & & \\
\hline \multicolumn{7}{|l|}{ Personal factors } \\
\hline Age, Mean (SD) & \multicolumn{2}{|c|}{$53.75(16.71)$} & \multicolumn{2}{|c|}{$55.43(17.60)$} & $<0.001$ & $* * *$ \\
\hline Female & 1,941 & 76.1 & 13,961 & 73.3 & 0.002 & $* *$ \\
\hline Age group & & & & & $<0.001$ & $* * *$ \\
\hline $18-34$ years & 328 & 12.9 & 2,509 & 13.2 & & \\
\hline $35-44$ years & 446 & 17.5 & 2,896 & 15.2 & & \\
\hline $45-54$ years & 568 & 22.3 & 3,786 & 19.9 & & \\
\hline 55-64 years & 536 & 21.0 & 3,576 & 18.8 & & \\
\hline$\geq 65$ years & 672 & 26.4 & 6,282 & 33.0 & & \\
\hline \multicolumn{3}{|c|}{ Medicare Advantage Enrollment } & & & \multicolumn{2}{|l|}{0.661} \\
\hline Commercial & 1,559 & 61.1 & 11,560 & 60.7 & & \\
\hline Medicare Advantage & 991 & 38.9 & 7,489 & 39.3 & & \\
\hline Insurance plan type & & & & & 0.598 & \\
\hline HMO & 792 & 31.1 & 6,101 & 32.0 & & \\
\hline $\mathrm{PPO} / \mathrm{POS}$ & 1,241 & 48.7 & 9,105 & 47.8 & & \\
\hline Other & 517 & 20.3 & 3,843 & 20.2 & & \\
\hline \multicolumn{7}{|l|}{ Environmental factor } \\
\hline Census region of residence & & & & & \multirow[t]{5}{*}{0.084} & \\
\hline Northeast & 268 & 10.5 & 1,733 & 9.1 & & \\
\hline Midwest & 647 & 25.4 & 4,716 & 24.8 & & \\
\hline South & 1,002 & 39.3 & 7,682 & 40.3 & & \\
\hline West & 633 & 24.8 & 4,918 & 25.8 & & \\
\hline \multicolumn{7}{|l|}{ Index Year } \\
\hline Years 2008-2010 & 750 & 29.4 & 5,521 & 29 & \multirow[t]{3}{*}{0.740} & \\
\hline Years 2011-2013 & 847 & 33.2 & 6,258 & 32.9 & & \\
\hline Years 2014-2015 & 953 & 37.4 & 7,270 & 38.2 & & \\
\hline \multicolumn{7}{|c|}{ Health and illness related factors } \\
\hline \multicolumn{7}{|l|}{ Type of CNPC } \\
\hline Back/Neck Pain (yes) & 1,933 & 75.8 & 13,435 & 70.5 & $<0.001$ & $* * *$ \\
\hline Arthritis/Joint Pain(yes) & 2,353 & 92.3 & 17,375 & 91.2 & 0.073 & \\
\hline Headache/Migraine (yes) & 991 & 38.9 & 6,144 & 32.3 & $<0.001$ & $* * *$ \\
\hline $\begin{array}{l}\text { Neuropathic/other } \\
\text { chronic pain (yes) }\end{array}$ & 701 & 27.5 & 4,073 & 21.4 & $<0.001$ & $* * *$ \\
\hline No. of CNPC a & & & & & $<0.001$ & $* * *$ \\
\hline 1 & 453 & 17.8 & 4,360 & 22.9 & & \\
\hline 2 & 1,042 & 40.9 & 8,562 & 44.9 & & \\
\hline
\end{tabular}




\begin{tabular}{|c|c|c|c|c|c|c|}
\hline$\geq 3$ & 1,055 & 41.4 & 6,127 & 32.2 & \multirow{2}{*}{\multicolumn{2}{|c|}{0.380}} \\
\hline \multicolumn{5}{|c|}{ Number of physical chronic conditions ${ }^{b}$} & & \\
\hline & 1,028 & 40.3 & 7,919 & 41.6 & & \\
\hline 1 to 2 & 898 & 35.2 & 6,668 & 35.0 & & \\
\hline 3 & 624 & 24.5 & 4,462 & 23.4 & & \\
\hline Anxiety disorders(yes) & 940 & 36.9 & 5,047 & 26.5 & $<0.001$ & \\
\hline Sleep disorders(yes) & 763 & 29.9 & 4,194 & 22.0 & $<0.001$ & \\
\hline Obesity diagnosis (yes) & 430 & 16.9 & 2,936 & 15.4 & 0.058 & \\
\hline \multicolumn{5}{|l|}{ Polypharmacy $^{c}$} & $<0.001$ & \\
\hline Yes & 1,507 & 59.1 & 8,261 & 43.4 & & \\
\hline No & 1,043 & 40.9 & 10,788 & 56.6 & & \\
\hline \multicolumn{7}{|c|}{ Commonly used medications ${ }^{c}$} \\
\hline NSAIDS use & 717 & 28.1 & 4,859 & 25.5 & 0.005 & \\
\hline Benzodiazepine use & 961 & 37.7 & 5,078 & 26.7 & $<0.001$ & \\
\hline \multicolumn{7}{|c|}{ 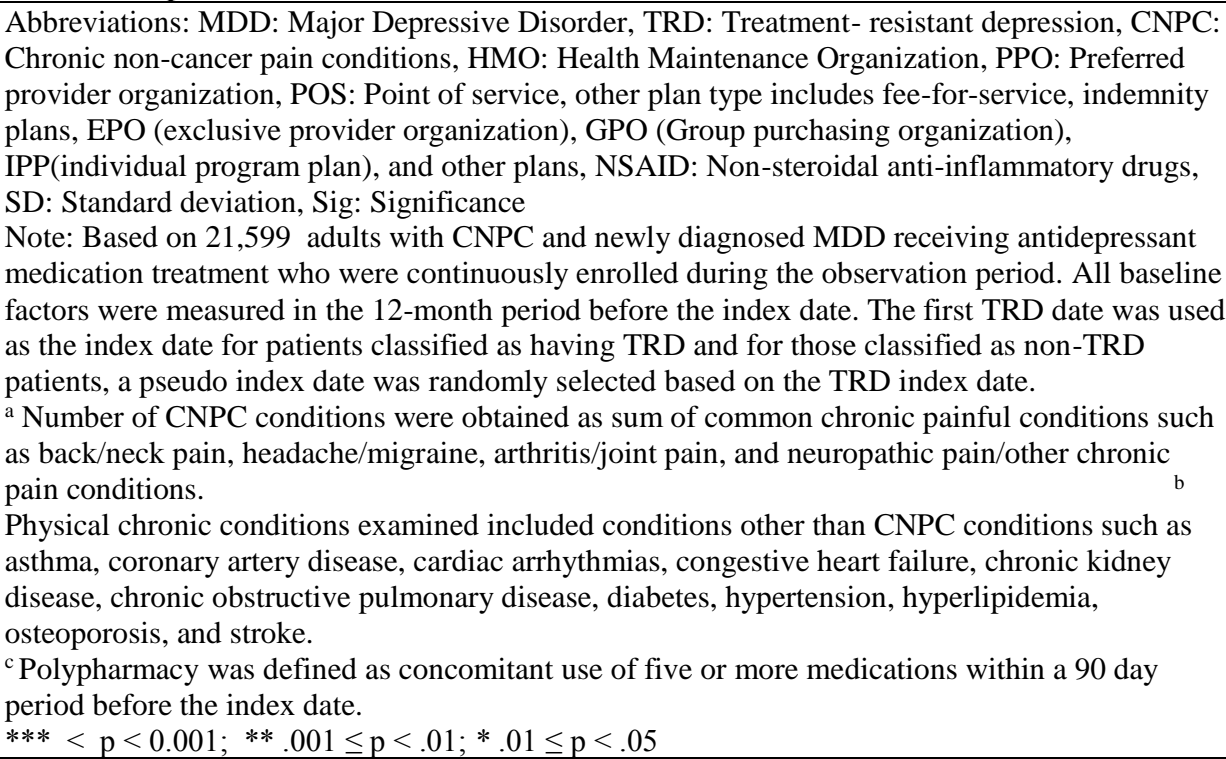 } \\
\hline
\end{tabular}




\section{Appendix 7.12. Chapter 4. Percentage of LTOT use (defined as continuous opioid supply for >120 days) over time for all adults and by TRD status among adults with CNPC and newly diagnosed MDD}

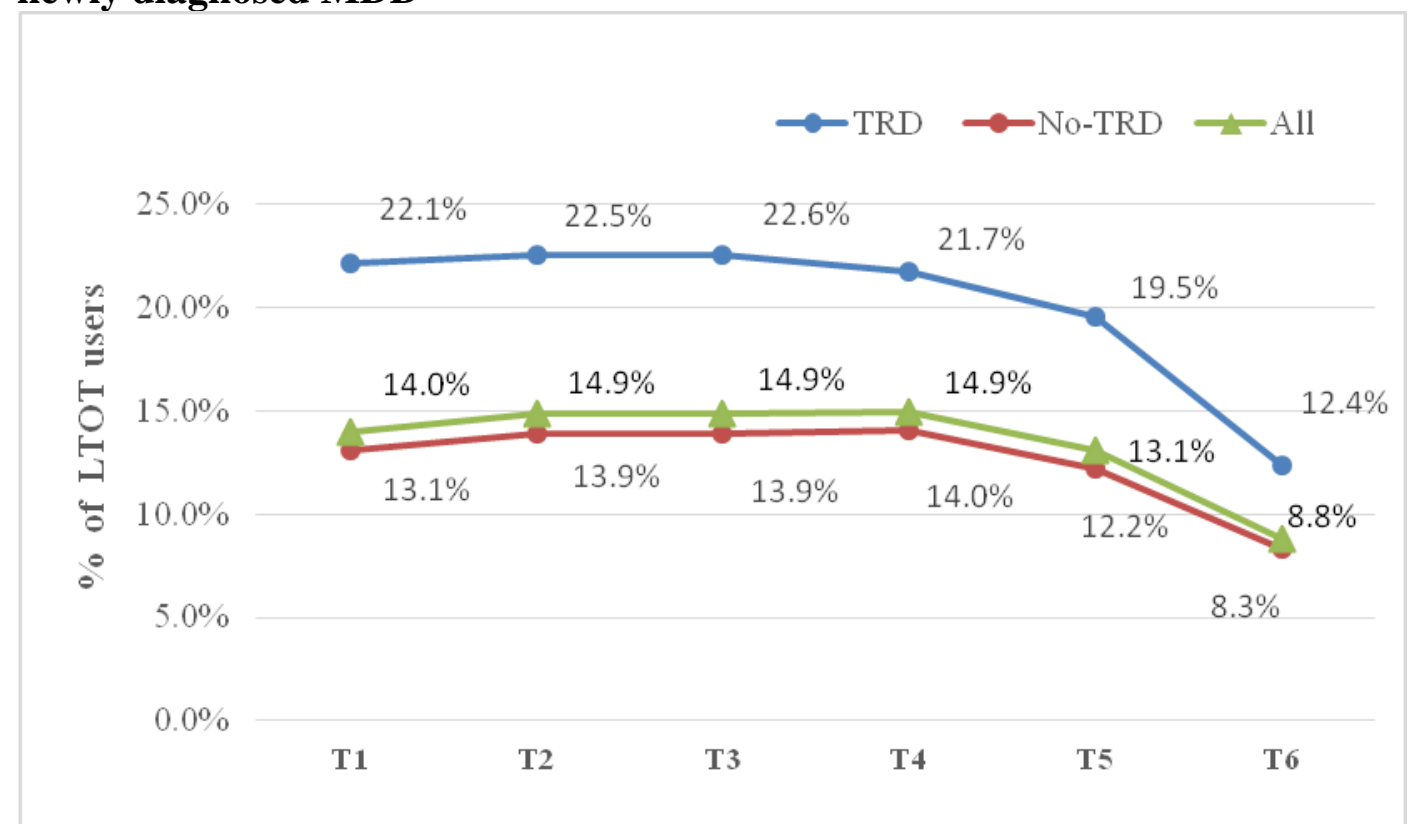

Abbreviations: MDD: Major Depressive Disorder, TRD: Treatment- resistant depression, CNPC: Chronic non-cancer pain conditions, LTOT: Long-term opioid therapy.

LTOT was defined as at least a $>90$ days of continuous supply of prescription opioids within a 180-day period.

$P$ value for percentage of LTOT use over time for all adults, adults with TRD and without TRD was $<0.001$

P-values were obtained from unadjusted Generalized estimating equation models. 


\begin{tabular}{|c|c|c|c|c|}
\hline \multicolumn{5}{|c|}{$\begin{array}{l}\text { Appendix 7.13. Chapter4. Unadjusted and Adjusted Odds Ratio and 95\% Confidence } \\
\text { Interval from Generalized Estimating Equation (GEE) of TRD on Long -term opioid } \\
\text { therapy (defined as continuous opioid supply for }>120 \text { days) among } \\
\text { Adults with CNPC and Newly Diagnosed MDD } \\
\text { Optum Clinformatics Real-World De-identified Data, 2007-2017 }\end{array}$} \\
\hline $\begin{array}{r}\text { Parameter } \\
\text { estimate }(\beta)\end{array}$ & SE & OR & $95 \% \mathrm{CI}$ & P Value \\
\hline \multicolumn{5}{|c|}{ Model 1: Unadjusted association between time and LTOT } \\
\hline-0.056 & 0.003 & - & - & $<0.001$ \\
\hline \multicolumn{5}{|c|}{ Model 2: Unadjusted Association of time and TRD with LTOT } \\
\hline \multicolumn{5}{|l|}{ TRD (Ref: No TRD) } \\
\hline 0.56 & 0.045 & 1.75 & {$[1.60,1.91]$} & $<0.001$ \\
\hline-0.057 & 0.004 & - & - & \\
\hline \multicolumn{5}{|c|}{$\begin{array}{c}\text { Model 2: Unadjusted Association of time, TRD, and interaction of time and TRD with } \\
\text { LTOT }\end{array}$} \\
\hline \multicolumn{5}{|l|}{ TRD (Ref: No TRD) } \\
\hline 0.63 & 0.052 & 1.87 & {$[1.68,2.07]$} & $<0.001$ \\
\hline-0.052 & 0.004 & - & - & $<0.001$ \\
\hline TRD*Time & 0.011 & - & - & 0.011 \\
\hline \multicolumn{5}{|c|}{ Model 3: Adjusting for personal, environmental and health/illness related factors ${ }^{\text {a }}$} \\
\hline Characteristics & & AOR & $95 \%$ CI & P Value \\
\hline \multicolumn{5}{|l|}{ TRD (Ref: No TRD) } \\
\hline 0.45 & 0.055 & 1.57 & {$[1.41,1.75]$} & $<0.001$ \\
\hline-0.049 & 0.004 & - & - & $<0.001$ \\
\hline TRD*Time & 0.013 & - & - & 0.001 \\
\hline \multicolumn{5}{|c|}{$\begin{array}{l}\text { Abbreviations: MDD: Major Depressive Disorder, TRD: Treatment- resistant depression, CNPC: Chronic } \\
\text { non-cancer pain conditions, NSAID: Non-steroidal anti-inflammatory drugs, OR: Odds ratio, AOR: } \\
\text { Adjusted odds ratio, CI: Confidence Interval } \\
\text { Note: Based on 21,599 adults with CNPC and newly diagnosed MDD receiving antidepressant medication } \\
\text { treatment who were continuously enrolled during the observation period. } \\
\text { Long-term opioid therapy was defined as at least a > } 120 \text { days of continuous supply of prescription opioids } \\
\text { within a 180-day period } \\
\text { a Only selected significant variables are presented. The fully adjusted model adjusted for all personal } \\
\text { factors (age, sex, insurance plan type, Medicare Advantage Enrollment), environmental factors (region of } \\
\text { residence, index year), and health and illness factors (number of physical chronic conditions, number of } \\
\text { CNPC, presence of anxiety disorders, sleep disorders, obesity, and medication use (polypharmacy, } \\
\text { NSAID, and benzodiazepine use). Polypharmacy, NSAIDs and benzodiazepines used as time varying } \\
\text { variables and were measured at all six time points. } \\
\text { b Number of CNPC conditions were obtained as sum of common chronic painful conditions such as } \\
\text { back/neck pain, headache/migraine, arthritis/joint pain, and neuropathic pain/other chronic pain conditions. } \\
\text { c Physical chronic conditions examined included conditions other than CNPC conditions such as asthma, } \\
\text { coronary artery disease, cardiac arrhythmias, congestive heart failure, chronic kidney disease, chronic } \\
\text { obstructive pulmonary disease, diabetes, hypertension, hyperlipidemia, osteoporosis, and stroke. }\end{array}$} \\
\hline
\end{tabular}

